\title{
Constraining stellar evolution theory with asteroseismology of $\gamma$ Doradus stars using deep learning
}

\section{Stellar masses, ages, and core-boundary mixing}

\author{
J.S.G. Mombarg ${ }^{1}$, T. Van Reeth ${ }^{1}$, and C. Aerts ${ }^{1,2,3}$ \\ 1 Institute of Astronomy, KU Leuven, Celestijnenlaan 200D, B-3001 Leuven, Belgium \\ e-mail: joey.mombarg@kuleuven. be \\ 2 Department of Astrophysics, IMAPP, Radboud University Nijmegen, PO Box 9010, 6500 GL Nijmegen, The Netherlands \\ 3 Max Planck Institute for Astronomy, Koenigstuhl 17, 69117 Heidelberg, Germany \\ Received September, 28 2020; accepted March, 192021
}

\begin{abstract}
Context. The efficiency of the transport of angular momentum and chemical elements inside intermediate-mass stars lacks proper calibration, thereby introducing uncertainties on a star's evolutionary pathway. Improvements require better estimation of stellar masses, evolutionary stages, and internal mixing properties.

Aims. We aim to develop a neural network approach for asteroseismic modelling and test its capacity to provide stellar masses, ages, and overshooting parameter for a sample of $37 \gamma$ Doradus stars for which these parameters were previously determined from their effective temperature, surface gravity, near-core rotation frequency, and buoyancy travel time, $\Pi_{0}$. Here, our goal is to perform the parameter estimation from modelling of individual periods measured for dipole modes with consecutive radial order rather than from $\Pi_{0}$. We assess if fitting of these individual mode periods increases the capacity of the parameter estimation.

Methods. We have trained neural networks to predict theoretical pulsation periods of high-order gravity modes $(n \in[15,91])$, as well as the luminosity, effective temperature, and surface gravity for a given mass, age, overshooting parameter, diffusive envelope mixing, metallicity, and near-core rotation frequency. We have applied our neural networks for Computing Pulsation Periods and Photospheric Observables, C-3P0, to our sample and compute grids of stellar pulsation models for the estimated parameters.

Results. We present the near-core rotation rates (from literature) as a function of the inferred stellar age and critical rotation rate. We assess the rotation rates of the sample near the start of the main sequence assuming rigid rotation. Furthermore, we measure the extent of the core overshoot region and find no correlation with mass, age, or rotation. Finally, for one star in our sample, KIC 12066947, we find indications of mode coupling in the period spacing pattern which we cannot reproduce with mode trapping.

Conclusions. The neural network approach developed in this study allows for the derivation of stellar properties dominant for stellar evolution - such as mass, age, and extent of core-boundary mixing. It also opens a path for future estimation of mixing profiles throughout the radiative envelope, with the aim to infer those profiles for large samples of $\gamma$ Doradus stars.
\end{abstract}

Key words. asteroseismology - stars: evolution - stars: oscillations (including pulsations) - stars: rotation - stars: interiors

\section{Introduction}

Accurate predictions of a star's evolutionary path depend on the accuracy of the description of transport of angular momentum (AM, e.g. Maeder 2009; Aerts et al. 2019) and chemical elements (e.g. Salaris \& Cassisi 2017). The transport mechanisms are still poorly understood, thereby introducing uncertainties in state-of-the-art stellar structure and evolution models. These uncertainties already occur during the core-hydrogen burning stage, and hence propagate into models of more evolved stars as well. Asteroseismology, the study of stellar oscillations, of lowto-intermediate mass $M_{\star} \lesssim 3.3 \mathrm{M}_{\odot}$ stars constitutes a powerful tool to measure surface- and near-core rotation rates across various evolutionary phases: main sequence (MS); Kurtz et al. 2014; Saio et al. 2015; Van Reeth et al. 2016, 2018; Christophe et al. 2018; Ouazzani et al. 2017, subgiants and red giants; Beck et al. 2012; Mosser et al. 2012; Gehan et al. 2018, and white dwarfs; Hermes et al. 2017. The empirically derived rotation rates require the transport of AM to be one to two order(s) of magnitude more efficient than what is currently predicted by theory (e.g.
Cantiello et al. 2014; Fuller et al. 2014; Eggenberger et al. 2017; Tayar \& Pinsonneault 2018; Eggenberger et al. 2019a,b; Fuller et al. 2019; den Hartogh et al. 2019, 2020). It has been suggested by Eggenberger et al. (2017) that the efficiency of AM transport increases with increasing mass.

Aerts et al. (2019) (their Figure 4) present an overview of measured core rotation rates versus the surface gravity $(\log g)$ in the literature for stars with $M_{\star} \in[0.72,7.9] \mathrm{M}_{\odot}$. While the different evolutionary stages can be distinguished based on $\log g$, the typical uncertainty is too large to infer any correlations between the rotation rate and the stellar age (Aerts et al. 2017). Instead of using $\log g$ as an age proxy, Ouazzani et al. (2019) used the reduced asymptotic period spacing $\Pi_{0}$, which represents the buoyancy travel time throughout the star, as derived for a sample of $\gamma$ Doradus ( $\gamma$ Dor) stars. Such stars are of spectral type late-A to early-F $\left(1.4 \mathrm{M}_{\odot} \lesssim M_{\star} \lesssim 1.9 \mathrm{M}_{\odot}\right)$ and show gravity $(\mathrm{g})$ modes excited via a convective flux blocking mechanism (e.g. Guzik et al. 2000; Dupret et al. 2005), although the $\kappa$ mechanism also plays a role for the hotter members of the class (Xiong et al. 2016). 
In a chemically homogeneous, non-rotating, non-magnetic star, the periods of $\mathrm{g}$ modes - with the same spherical degree $\ell$ and azimuthal order $m$, but consecutive radial order $n-$ are equally spaced in period in the asymptotic regime $(n \gg \ell)$, namely by $\Pi_{0} / \sqrt{\ell(\ell+1)}$. It is therefore customary to present the oscillations in a period spacing diagram where the spacing $\Delta P=P_{n+1}-P_{n}$ of each individual mode with radial order $n$ is plotted as a function of its period $P_{n}$. The advent of the Kepler (Borucki et al. 2010) and TESS (Ricker et al. 2015) missions have led to numerous detections of such period spacing patterns in $\gamma$ Dor stars (Kurtz et al. 2014; Saio et al. 2015; Van Reeth et al. 2015a; Keen et al. 2015; Saio et al. 2018; Li et al. 2019a,b; Antoci et al. 2019). A first attempt at estimating stellar masses and ages of a sample of $37 \gamma$ Dor stars was done by Mombarg et al. (2019), using $\Pi_{0}, T_{\text {eff }}$, and $\log g$ assembled in Van Reeth et al. (2015b, 2016) as input for the modelling. Their results show that faster rotating stars tend to be in the early phases of the MS and there was no correlation between age and the detection of Rossby modes. In this paper we aim to refine their work by fitting the measured periods for each of the individual dipole modes with consecutive radial order instead of just $\Pi_{0}$ as asteroseismic observable.

Besides probing stellar rotation rates, asteroseismology also allows for the scrutiny of a star's internal chemical mixing profile (e.g. Pedersen et al. 2018; Michielsen et al. 2019; Pedersen et al. 2021). During the core-hydrogen burning phase, a chemical gradient is introduced in the near-core region as the mean molecular weight inside the core increases.

The presence of a chemical gradient causes mode trapping, which translates into characteristic dips in the period spacing pattern (Miglio et al. 2008). Yet, mixing occurs in the core boundary layers, due to effects such as core overshooting and a variety of phenomena occurring at the bottom of the envelope. In addition, mixing throughout the radiative envelope occurs, for example as a result of shear instabilities (Maeder 2009) or internal gravity waves (e.g. Rogers \& McElwaine 2017). Such forms of mixing alter the chemical gradient, making it possible to probe the efficiency of the mixing throughout the stellar envelope and infer properties of the mixing profile provided that modes with suitable probing power can be detected and identified (Aerts 2021). This has been achieved for a sample of Btype g-mode pulsators, revealing a large range of mixing levels ranging from $\sim 10$ to $\sim 10^{6} \mathrm{~cm}^{2} \mathrm{~s}^{-1}$ (Pedersen et al. 2021). For $\gamma$ Dor stars, our understanding of mixing in the envelope is less advanced because their levels of mixing at the deep bottom of the envelope, at the interface with the core overshoot zone, were found to be much lower (Van Reeth et al. 2016; Mombarg et al. 2019).

In this paper, we take the first steps to develop a new modelling approach based on deep learning, with the future aim to estimate the mixing profile throughout the radiative envelope of $\gamma$ Dor stars. In this initial study, we only treat the mixing in the near-core boundary layer, while fixing the one in the outer envelope. We do this because we first aim to assess the precision estimation of the global stellar parameters, such as the mass, metallicity, age, and convective-core overshooting for an exponential overshooting prescription, by relying on the individual mode period spacings rather than on just $\Pi_{0}$. In order to test the capacity of our new deep learning method, we re-model the measured period spacing patterns of the $37 \gamma$ Dor stars from the sample of Van Reeth et al. (2015b) and derive masses, ages, and nearcore mixing efficiencies. These are then compared to those obtained earlier by Mombarg et al. (2019). If we achieve a better modelling strategy based on our initial deep learning approach, then future applications in a much higher-dimensional parameter space become possible, which would allow for the additional estimation of the envelope mixing profiles responsible for the observed morphologies of the period spacing patterns in terms of isolated or recurring dips as observed by (Van Reeth et al. 2015b; Li et al. 2019a,b).

\section{Deep Learning}

One of the biggest challenges in asteroseismic modelling of period spacing patterns is that it requires a parameter search in a high-dimensional space. The most important parameters constitute the stellar mass $\left(M_{\star}\right)$, the initial metallicity $Z$, and the hydrogen mass fraction in the convective core $\left(X_{\mathrm{c}}\right.$; a proxy for the stellar age). In addition, the chemical mixing profile is typically split in two parts; the convective core overshooting (darkcoloured regions in Figure 1) and the mixing efficiency in the radiative envelope (denoted as $D_{0}$ in Figure 1). Given that the physical mechanisms at stake are still unknown, both the overshoot and envelope mixing profile are parameterized by a function dependent on the local radius $r$, where the exact functional description in real stars is still a matter of debate (e.g., Aerts 2021, for a general discussion and example profiles). Mombarg et al. (2019) showed that diffusive exponentially decaying core overshooting cannot be distinguished from convective penetration in their modelling based on $\Pi_{0}$. As our main aim here is to evaluate our new deep learning modelling method in terms of capacity to estimate the global parameters and not to investigate the detailed morphology of the period spacing patterns, we rely on an exponentially decaying diffusive core-overshooting prescription. Our focus thus lies on the parameter estimation delivered by the individual period spacing values coupled to a neural network. The diffusive overshooting is expressed as a dimensionless parameter $f_{\text {ov }}$ times the pressure scale height (Freytag et al. 1996):

$D_{\mathrm{CBM}}(r)=D_{\mathrm{CBM}}\left(r_{0}\right) \exp \left(\frac{-2\left(r-r_{0}\right)}{f_{\mathrm{ov}} H_{P}\left(r_{\mathrm{cc}}\right)}\right)$,

where $r_{\mathrm{cc}}$ and $H_{P}$ are the distance from the stellar centre to the (Schwarzschild) core boundary, and pressure scale height, respectively. For numerical purposes, the mixing efficiency at the stitching point between convective mixing in the core and the core overshoot mixing is evaluated at $r_{\mathrm{cc}}-f_{0} H_{P}\left(r_{\mathrm{cc}}\right)$, for which we set $f_{0}=0.005$. As said, we do not focus on the shape of the mixing profile in the outer radiative envelope in this first application of the neural network. Hence we assume a constant mixing efficiency throughout the radiative zone for simplicity.

Aside from these most important parameters determining the stellar structure and evolution (SSE) models, $M_{\star}, Z, X_{\mathrm{c}}$, and $f_{\mathrm{ov}}$, we introduce the internal rotation frequency $f_{\text {rot }}$ at the level of the pulsation computations. Hence, asteroseismic modelling of stars with a convective core is in general a $+6 \mathrm{D}$ problem (Aerts et al. 2018; Aerts 2021). This modelling scheme becomes rapidly computationally expensive with the option to also assess mixing profiles via free parameters and for application to large $\gamma$ Dor sample sizes such as the one provided by Li et al. (2020). To deal with such types of future applications, grids of stellar or pulsation models can be approximated and replaced by statistical models (Mombarg et al. (2019); Pedersen et al. (2021)) or neural networks (this work). The latter method has been applied to non-rotating pulsation models for a benchmark sample of pulsating B-type stars by Hendriks \& Aerts (2019), reaching an accuracy of $\sim 10 \%$ on the pulsation frequencies predicted by deep 


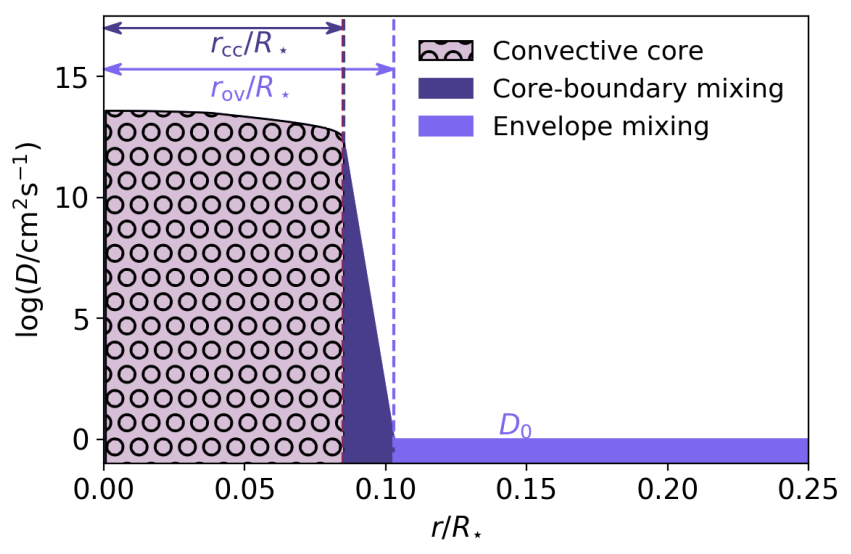

Fig. 1. Chemical mixing profile showing the different mixing zones in a model for an intermediate-mass star. The ordinate shows the local efficiency of chemical (diffusive) mixing. The thin outer convection zone is not shown in the plot. The radii of the convective core boundary $\left(r_{\mathrm{cc}}\right)$ and overshoot zone $\left(r_{\mathrm{ov}}\right)$ are also indicated. Mixing inside the convective regions is based on MLT and occurs instantaneously. The red dashed line slightly to the left of $r_{\mathrm{cc}} / R_{\star}$ indicates the starting point of the overshoot zone ( $r_{0}$ in Eq. 1).

learning models, compared to those from the pulsation models. The observed values for $Z$ and $f_{\text {rot }}$ show that the stars in our sample cover a large range. Furthermore, the $\gamma$ Dor instability strip is narrow, compared to those of other pulsators. As such, properly covering the most important stellar parameters with an equally-spaced linear grid set-up, as is commonly done in asteroseismic modelling, would require a very large amount of equilibrium models. We seek to circumvent this by replacing the need of such extensive model grids by a neural network (NN). This study serves as an initial proof-of-concept study of the application of deep learning to asteroseismic modelling of g-mode pulsators, with the idea that future studies may rely on a larger set of grids with different input physics.

\subsection{Predicting pulsation periods}

In this work, we train a neural network to predict g-mode periods of theoretical pulsation models for a given $M_{\star}, X_{\mathrm{c}}, Z, f_{\mathrm{ov}}$, $D_{0}$, and $f_{\text {rot }}$. We have chosen this approach instead of using the asteroseismic data as input and the stellar parameters as output (e.g. Hon et al. 2020), because this allows for more flexibility in the number of free parameters, as we can fix some of these parameters without having to retrain the NN. We keep this outlook in mind for future applications based on more complex envelope mixing profiles. In this initial study, we compute a grid of stellar equilibrium models for the parameter ranges listed in Table 1, based on the ranges used in Mombarg et al. (2019). We base the range of $f_{\text {ov }}$ on the typical values found by Claret $\&$ Torres (2017) for the appropriate mass regime. The parameter space is sampled both linear, and quasi random from a Sobol sequence, following Bellinger et al. (2016). This way, we obtain a high sampling density for each parameter whilst also allowing the parameters to vary independently. The stellar equilibrium models have been computed with the SSE code MESA (r11701) (Paxton et al. 2011, 2013, 2015, 2018, 2019) for the computations of the stellar equilibrium models, using the same input physics as the models without atomic diffusion described in Mombarg et al. (2020). The stars in our sample are assumed to have a chemical mixture similar to the Sun, that is, we take the

\begin{tabular}{lll}
\hline \hline Parameter & $\begin{array}{l}\text { Lower } \\
\text { Boundary }\end{array}$ & $\begin{array}{l}\text { Upper } \\
\text { Boundary }\end{array}$ \\
\hline$M_{\star}\left(\mathrm{M}_{\odot}\right)$ & 1.3 & 2.0 \\
$X_{\mathrm{c}}$ & 0.05 & 0.70 \\
$Z$ & 0.011 & 0.023 \\
$f_{\text {ov }}$ & 0.010 & 0.030 \\
$D_{0}\left(\mathrm{~cm}^{2} \mathrm{~s}^{-1}\right)$ & 1 & 100 \\
$f_{\text {rot }}\left(\mathrm{d}^{-1}\right)$ & 0.0 & 2.3 \\
\hline
\end{tabular}

Table 1. Extent of the grid of stellar models used to train the C-3P0 neural network.

Solar abundances from Asplund et al. (2009), and scale these according to the metallicity, $Z$. The helium mass fraction of the models are set according to the enrichment rate found by Verma et al. (2019) and the initial hydrogen mass fraction is set by $X_{\text {ini }}=1-Y_{\text {ini }}-Z=0.756-2.226 Z$ with ${ }^{2} \mathrm{H} /{ }^{1} \mathrm{H}$ and ${ }^{3} \mathrm{He} /{ }^{4} \mathrm{He}$ isotope ratios set to the values measured by Asplund et al. (2009).

From the stellar equilibrium models, the theoretically predicted pulsations are computed with the pulsation code GYRE (v5.2; Townsend \& Teitler 2013; Townsend et al. 2018), using the adiabatic framework and treating the Coriolis acceleration non-perturbatively. The equations of motion are decoupled in the angular and radial components by neglecting the latitudinal component of the rotation vector (Traditional Approximation of Rotation; TAR; Eckart 1960; Townsend 2003). Analyses of large samples of $\gamma$ Dor stars by Van Reeth et al. (2016) and Li et al. (2019b) reveal the prograde dipole mode $(\ell=1, m=1)$ is by far the most observed mode geometry. Hence, for each stellar equilibrium model we compute the predicted pulsation frequencies for $(\ell, m)=(1,1)$ modes and radial orders $n \in[15,91]$ (thus $\Delta P_{n}$ for $n \in[15,90]$ ), using the pulsation code GYRE (Townsend 2003; Townsend \& Teitler 2013). The range of radial orders is based on the distribution found by Li et al. (2020), taking the most ubiquitous ones. Again, we quasi-randomly sample $X_{\mathrm{c}}$ and $f_{\text {rot }}$ within the ranges listed in Table 1 . The rotation frequency range is based on the minimum and maximum observed values in the sample of Van Reeth et al. (2016). The total output data set contains 38915 pulsation models, of which $70 \%$ is used for training and $30 \%$ for validation.

Neural networks are powerful numerical methods suitable to treat multi-dimensional and complex regression problems (see Bishop 1995; Glorot et al. 2011, to which we refer the reader for an introduction and general overview on deep learning). We constructed a dense NN comprising five layers, using the KERAS package for Python (with Tensorflow 1.14.0), for which the schematic overview of the $\mathrm{NN}$ is shown in Figure 2. A rectified linear unit (ReLU) was used as activation function for the neurons of the first four layers, and a linear activation function for the output layer, including a bias term for both activation functions. We have tested several of the most commonly used activation functions (ReLU, sigmoid, tanh), for which we found that the ReLU activation functions yielded the best performance for our NN. The output of the NN are the predicted periods of radials orders $n \in[15,91]$.

Similarly to the construction of statistical models as a means to represent reality, adding nonlinear combinations of the input parameters, may increase the performance of a NN. Inspired by the regressions performed in Mombarg et al. (2019), we found the performance of our NN to be enhanced by adding two product terms of the most correlated parameters, the mass and age, and mass and metallicity, to the input vector $\theta=$ $\left(M_{\star}, X_{\mathrm{c}}, Z, f_{\mathrm{ov}}, D_{0}, f_{\mathrm{rot}}, M_{\star} \cdot X_{\mathrm{c}}, M_{\star} \cdot Z\right)$. Each component of $\theta$ 
is normalized as per,

$\bar{\theta}_{j}^{i}=\frac{\theta_{j}^{i}-\min \theta^{i}}{\max \theta^{i}-\min \theta^{i}}$,

where $i$ is the parameter index and $j$ the model index. The technique of 'early stopping' is applied to prevent the loss from increasing again after several epochs, that is, the training is terminated when the validation loss has not decreased during three consecutive epochs. The weights are then restored to the configuration for which the validation loss was the lowest.

The problem of overfitting is common when training a NN, that is, the $\mathrm{NN}$ achieves a high precision on the training set while it fails to generalize the correlations, and thus underperforms on the validation set. To remedy overfitting, a penalty term $\lambda \sum_{j} w_{j}^{2}$ is added to the loss function, where $w_{j}$ are the synaptic weights and $\lambda$ the control parameter, which we set to 0.01 . This technique penalizes for large weights and is commonly referred to as L2 or ridge regularization (cf. Bishop 1995, Chap. 9.2). As a loss function, the mean squared error is used. The final configuration of the weights is somewhat dependent on the initial weights, which introduces noise in the predictions. To remedy this, we train six NNs with different initial weights and average their predictions (this technique is often referred to as 'ensemble learning').

The so-called learning curve is a common diagnostic to assess whether a NN is able to make robust predictions. This curve shows the loss (which includes the L2 regularization term) as a function of epoch. In the top panel of Figure 3 we show the learning curves for all NNs trained to predict the pulsation periods. Ideally, a NN should obtain roughly the same loss on the training and validation set, which is indeed the case here. When ensemble learning is applied, a mean absolute error of $0.0024 \mathrm{~d}$ ( $207 \mathrm{~s}$ ) on the pulsation periods is obtained over all models used to train and validate the NN. Typical observed spacings between periods of consecutive radial order vary from several hundreds to several thousands of seconds. It is more instructive to compare the accuracy of the NN with typical spacings rather than observational uncertainties, as the accuracy of the state-of-the-art pulsation models is typical orders of magnitude worse than the uncertainties on the measured periods from Kepler light curves (e.g. Buysschaert et al. 2018; Mombarg et al. 2020).

\subsection{Lifting degeneracies}

The modelling of mode periods or period spacing patterns is prone the degeneracies, mainly between mass and age, and mass and metallicity (e.g. Moravveji et al. 2015; Mombarg et al. 2019, 2020). We apply the same methodology as Mombarg et al. (2020), where the best model is selected from models that are compatible with the effective temperature $\left(T_{\text {eff }}\right)$ and surface gravity $(\log g)$, measured from spectroscopy. In addition to these two parameters, the luminosity $\left(\log \left(L / \mathrm{L}_{\odot}\right)\right)$ derived from the Gaia DR2 distances $(D)$ measured by Bailer-Jones et al. (2018) is used:

$\log L / \mathrm{L}_{\odot}=-0.4\left(M_{V}-V_{\odot}-31.572+\left[\mathrm{BC}_{V}-\mathrm{BC}_{V, \odot}\right]\right)$,

where $M_{V}=m_{V}-5 \log (D / 10 \mathrm{pc})-3.3 E(B-V), V_{\odot}=-26.76$, and $\mathrm{BC}_{V, \odot}=-0.080$. Bolometric corrections $\left(\mathrm{BC}_{V}\right)$ are computed as per Torres (2010) and reddening corrections $E(B-V)$ are taken from the Bayerstar2019 extinction map (Green et al. 2018). From the extinction, the correction to the visual magnitude is calculated using $A_{V}=3.3 E(B-V)$. Figure 4 shows the position of all stars in our sample in a Hertzsprung-Russell diagram (see Table A in Appendix).

\begin{tabular}{lll}
\hline \hline Output & Configuration & Networks \\
\hline$P_{n}, n \in[15,91]$ & $700,700,20000,20000,86$ & 6 \\
$\log L / \mathrm{L}_{\odot}$ & $700,700,20000,20000,1$ & 1 \\
$\log T_{\text {eff }}, \log g$ & $700,700,20000,20000,2$ & 1 \\
\hline
\end{tabular}

Table 2. Summary of the network configuration of C-3P0. The second column lists the number of neurons for each layer.

In addition to the mode periods, we have trained two NNs to predict $\left(T_{\text {eff }}, \log g\right.$ ) and $\log L$, respectively, using the same configuration as the network shown in Figure 2. To train these NNs, we use all time steps (on the MS) computed by MESA as opposed to using only the time step for which pulsation models were computed, that is, the training set comprises $423691 \mathrm{vec}-$ tors and the validation set comprises 141231 vectors. In Figure 5 we compare the predictions of the NNs of these photospheric observables to MESA evolutionary tracks. We obtain mean absolute errors of $\bar{\epsilon}_{\log L / \mathrm{L}_{\odot}}=0.010, \bar{\epsilon}_{\log T_{\text {eff }}}=0.004$, and $\bar{\epsilon}_{\log g}=0.011 \mathrm{dex}$ on the complete data set (training plus validation). The models shown in this figure were not included in the training or validation set. There is no exact method to find the optimal configuration of a NN. We started from a similar network configuration as presented in Hendriks \& Aerts (2019), and experimented with different numbers of hidden layers, and the number of neurons per layer, by means of trial and error. Table 2 summarizes the ensemble of all the networks for Computing Pulsation Periods and Photospheric Observables, C-3P0.

\section{Asteroseismic modelling}

Until recently, g-mode modelling of single $\gamma$ Dor stars relied on $\left(\Pi_{0}, T_{\text {eff }}, \log g\right)$ as input (Mombarg et al. 2019). In the work by Mombarg et al. (2020), $\Pi_{0}$ is replaced with the individual mode periods as asteroseismic input for the modelling of two slowly rotating $\gamma$ Dor stars. Furthermore, these authors investigated whether $T_{\text {eff }}$ and $\log g$ should be added to the fit or used a posteriori to select models which are consistent within $n \sigma$ compared to the observations. As typically tens of excited modes of consecutive radial order are observed in a single star, the spectroscopic observables have little relative weight in the merit function. Therefore, we opt to use the $\log T_{\text {eff }}$ and $\log g$ to select the best-fitting model from a subset of models which are within the 2- $\sigma$ uncertainty ranges of these photospheric observables. Aerts et al. (2018) introduced the Mahalanobis distance (MD) as a merit function in asteroseismic modelling to account for the correlated nature of the observed periods, as well as the theoretical uncertainty of the modes. The MD takes the (co)variances of the input parameters into account such that the contours of equal MD are aligned with the principal components, whereas for the $\chi^{2}$ merit function contours of equal $\chi^{2}$ are aligned with the base vectors (free parameters). For a given vector $\mathbf{Y}^{\text {(th) }}$, containing the periods of the identified radial orders, and a vector $\mathbf{Y}^{(\mathrm{obs})}$ containing the observed periods, the MD is defined as

$\mathrm{MD}=\left(\mathbf{Y}^{(\mathrm{th})}-\mathbf{Y}^{(\mathrm{obs})}\right)^{\top}(\mathbf{V}+\boldsymbol{\Sigma})^{-1}\left(\mathbf{Y}^{(\mathrm{th})}-\mathbf{Y}^{(\mathrm{obs})}\right)$.

The matrix $\mathbf{V}$ is the (co)variance matrix and $\boldsymbol{\Sigma}$ is a diagonal matrix, constructed from the uncertainties on the predicted mode periods by the NN. These can be treated as aleatoric, as the residuals on the training/validation set follow a normal distribution (see Appendix C). Therefore, each uncertainty $\sigma_{P_{n}^{(\text {th }}}$, with $n$ the radial order, is taken to be the standard deviation of the residuals over the complete grid of pulsation models. The uncertainties of the predictions are typically 


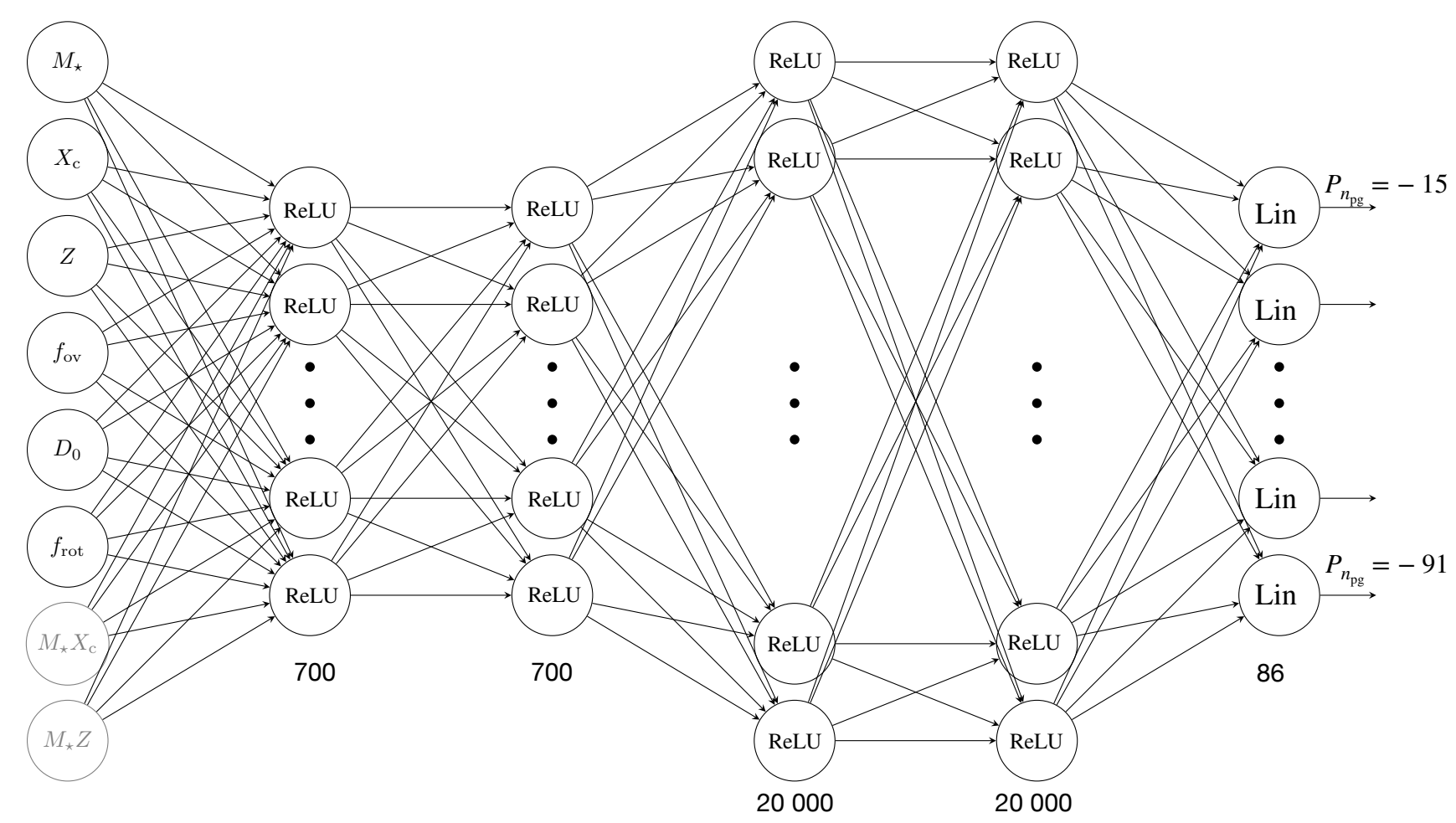

Fig. 2. Schematic overview of the configuration of the dense neural network trained to predict the periods of the $(\ell, m)=(1,1)$ modes for a given $\theta=\left(M_{\star}, X_{\mathrm{c}}, Z, f_{\mathrm{ov}}, D_{0}, f_{\mathrm{rot}}, M_{\star} \cdot X_{\mathrm{c}}, M_{\star} \cdot Z\right)$. The values of the input parameters are transformed as per Eq. (2).

two orders of magnitude larger than those from the observations.

The matching between the observed radial orders to those from a model is non trivial. Observed period spacing patterns of $\gamma$ Dor stars do not always form a single sequence of consecutive radial orders, but may be interrupted by missing modes. Therefore, the radial order matching in this paper is done as follows:

1. Start with the longest observed continuous sequence of $M$ periods and assign the shortest period $P_{0}^{(\mathrm{obs})}$ to the bestmatching period $P_{j, i}^{(\text {th })}$, as the shortest period has the smallest relative uncertainty.

2. Assign periods $P_{1}^{\text {(obs })}, \ldots, P_{M}^{(\mathrm{obs})}$ to the consecutive radial orders in the model.

3. Repeat step 1 for the second longest sequence, and so on. When a radial order is selected which has already been assigned to an observed period in one of the longer sequences, omit the model.

In this way, we account for the possibility of having multiple sequences of consecutive radial orders with missing modes in between those sequences. The construction of $\mathbf{V}$ can be done in two ways. 1: For each evaluated model $j$, each of the observed periods $P_{i}^{(\mathrm{obs})}$ is matched with a period $P_{i}^{\text {(th) }}$ corresponding to a radial order that may vary for different models. Matrix $V$ is given by,

$\mathbf{V}=\frac{1}{q-1} \sum_{j}^{q}\left(\mathbf{Y}_{j}^{(\mathrm{th})}-\overline{\mathbf{Y}}^{(\mathrm{th})}\right)\left(\mathbf{Y}_{j}^{(\mathrm{th})}-\overline{\mathbf{Y}}^{(\mathrm{th})}\right)^{\top}$,

where $q$ is the number of evaluated models, and

$\overline{\mathbf{Y}}^{(\mathrm{th})}=\frac{1}{q} \sum_{j}^{q} \mathbf{Y}_{j}^{(\mathrm{th})}$.
2: Take $\mathbf{Y}_{j}^{(\text {th) }}$ to contain the periods of all the radial orders predicted by the NN, that is, $n \in[15,91]$, and construct $\mathbf{V}$, thereby yielding a matrix with dimensions $77 \times 77$. Consecutively, for each model with radial order identifications $n_{\min }, \ldots, n_{\max }$ corresponding to the $N$ observed mode periods, remove the rows and columns of $\mathbf{V}$ belonging to the radial orders that were not identified in model $j$, yielding an $N \times N$ matrix, $\mathbf{V}_{j}^{\prime}$.

In this paper, we have used method (2) to compute the MD, such that the (co)variances do not depend on the radial order identification. This is the case for method (1), because the lowest period is matched first and the consecutive periods are then matched to the consecutive radial orders of the model. Hence, when the observed $\Pi_{0}$ does not match the $\Pi_{0}$ of the model, the discrepancy between the model and observations is larger for higher radial orders. Therefore the variance across the grid will also be larger for these radial orders, and by extension will be given a smaller weight. The maximum likelihood estimate (MLE) for our method is not equivalent to minimizing the merit function, as is the case for $\chi^{2}$. Instead, the likelihood of the observed data $\boldsymbol{D}$, given parameters $\boldsymbol{\theta}=\left(\theta^{1}, \theta^{2}, \theta^{3}\right)$, is given by,

$$
\begin{aligned}
L(\boldsymbol{\theta} \mid \boldsymbol{D}) & =P(\boldsymbol{D} \mid \boldsymbol{\theta}) \\
& =\exp \left(-\frac{1}{2}\left(\ln \left|\boldsymbol{V}^{\prime}\right|+\mathrm{MD}+k \ln (2 \pi)\right)\right),
\end{aligned}
$$

where $k=3$ is the number of free parameters. According to Bayes' theorem, the probability of component $\theta^{k}$ being within interval $\left[\theta_{a}^{k}, \theta_{b}^{k}\right]$ is given by

$$
\begin{aligned}
P\left(\theta_{a}^{k}<\theta^{k}<\theta_{b}^{k} \mid \boldsymbol{D}\right) & = \\
& \frac{\sum_{i}^{q} P\left(\boldsymbol{D} \mid \boldsymbol{\theta}_{i}\right) P\left(\theta_{i}^{1}\right) P\left(\theta_{i}^{2}\right) P\left(\theta_{i}^{3}\right)}{\sum_{j}^{Q} P\left(\boldsymbol{D} \mid \boldsymbol{\theta}_{j}\right) P\left(\theta_{j}^{1}\right) P\left(\theta_{j}^{2}\right) P\left(\theta_{j}^{3}\right)} .
\end{aligned}
$$



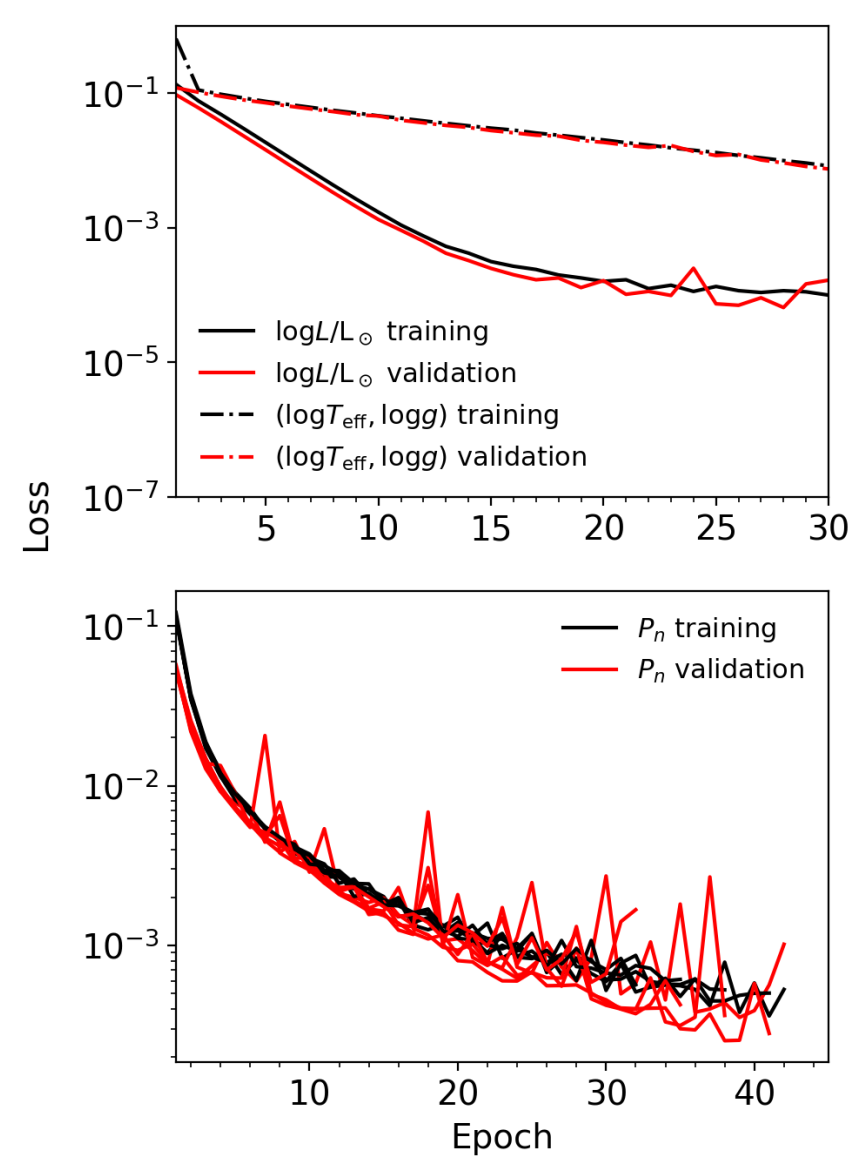

Fig. 3. Loss (mean squared error $+\mathrm{L} 2$ regularization term) as a function of epoch. The NNs training on the pulsation models (bottom panel) reached an optimal solution within the maximum number of epochs. In all cases similar loss is obtained on the training and validation sets, indicating the NNs were able to generalize the synaptic weights.

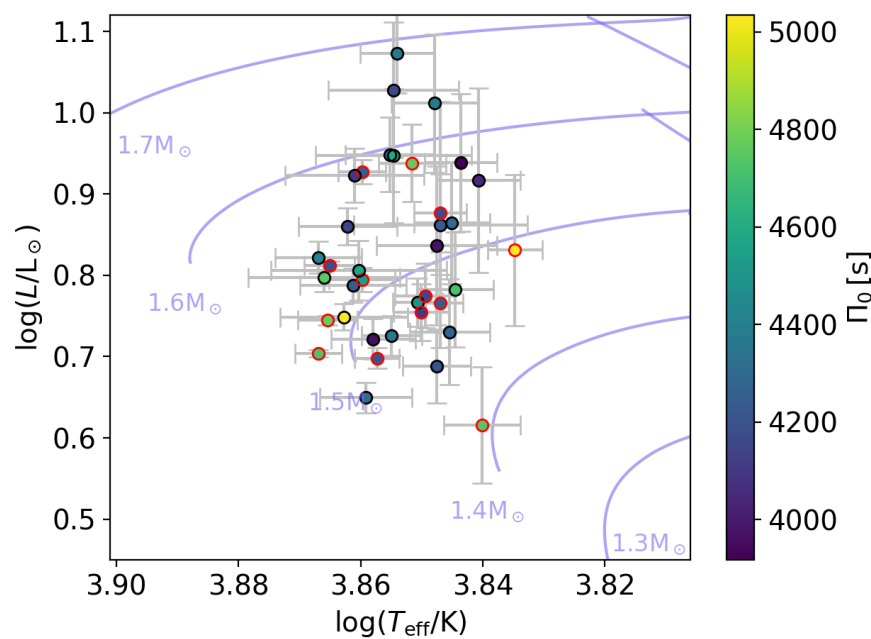

Fig. 4. Positions in the Hertzsprung-Russel diagram of all stars in our sample. The values of $T_{\text {eff }}$ and $\Pi_{0}$ are taken from Van Reeth et al. (2016). The evolution tracks shown are for $Z=0.014$ and $f_{\text {ov }}=0.0175$. For the stars marked with red circles no reliable extinction estimate could be made, because these are too close to the Sun, therefore, their interstellar extinction values are close to zero.

Article number, page 6 of 35
The sum over index $i$ is taken over the $q$ models with the highest likelihood such that $P\left(\theta_{a}^{k}<\theta^{k}<\theta_{b}^{k} \mid \boldsymbol{D}\right)=0.68$, where the sum over index $j$ is taken over all of the models that are consistent with the observed photospheric observables.

The modelling of $\mathrm{g}$ modes in $\gamma$ Dor stars is a highdimensional, degenerate problem. Optimizing all six aforementioned parameters at once will lead to noisy solution spaces, making uncertainty determination unwieldy. Yet, some parameters are more dominant than others, and thus, we first focus on the parameters which have the largest influence on the mode periods. Since the metallicity and near-core rotation rate have already been determined by Van Reeth et al. (2016), we fix these two parameters to the measured values. The influence of $f_{\mathrm{ov}}$, and specifically $D_{0}$ on the period spacings are mostly seen in the mode trapping. In this work, our aim is not to precisely model the trapped modes, but rather get an accurate fit to the global pattern of all observed modes. Accurately modelling trapped modes adds additional dimensionality to the problem in the form of structured (non-constant) mixing profiles (Pedersen et al. 2021), which we do not consider here. For each star in our sample, a best-fitting model is found as follows:

1. Randomly sample 5000 models in $\left(M_{\star}, X_{\mathrm{c}}, f_{\text {ov }}\right)$ with C-3P0, where $Z$ and $f_{\text {rot }}$ are fixed to the measured values, and $D_{0}=$ $1 \mathrm{~cm}^{2} \mathrm{~s}^{-1}$.

2. If some observed periods cannot be matched in $90 \%$ of the models (because the radial order is outside the range of $\mathrm{NN}$ ) remove either the shortest or the longest period depending on which one is outside the range. Repeat until a model can be matched.

3. From these 5000 models, get the minimum and maximum values of $M_{\star}$ and $X_{\mathrm{c}}$ such that the predicted $\log \left(L / \mathrm{L}_{\odot}\right), \log T_{\text {eff }}$, and $\log g$ are consistent within $2-\sigma$ of the respective uncertainties.

4. Randomly sample 15000 models, but now within the mass and $X_{\mathrm{c}}$ ranges obtained in the previous step. The solution and uncertainties are computed according to Eqs 7 and 8.

5. Since the exact periods of trapped modes are difficult for a NN to predict, we optimize the overshoot parameter with GYRE models once we have a good estimate of $\left(M_{\star}, X_{\mathrm{c}}, f_{\mathrm{ov}}\right)$ from the previous steps. We compute GYRE models for $f_{\text {ov }} \in[0.005,0.010,0,015,0.020,0.025,0.030,0.035]$ for the MLEs for $M_{\star}$ and $X_{\mathrm{c}}$ and for the upper/lower limits of these two parameters (63 models per star in total). Models rotating faster than the critical Roche frequency, $\Omega_{\text {crit,Roche }}=$ $\sqrt{\left(8 G M_{\star}\right) /\left(27 R_{\star}^{3}\right)}$, are not taken into account.

In the latter step, a $\chi^{2}$ merit function is used instead of the MD, as scanning such a small parameter range results in (co)variance matrices with very high condition numbers. The uncertainties on $f_{\text {ov }}$ are determined by,

$\chi_{1 \sigma}^{2}=\chi_{\min }^{2}\left(1+\sqrt{\frac{2}{N-k}}\right)$.

For stars where an additional mode geometry has been observed, the corresponding pulsation models are also computed and fitted simultaneously with the prograde dipole modes in Step 5.

\subsection{Theoretical benchmark}

First, we demonstrate the capabilities of C-3PO by applying the $\mathrm{NN}$ to a set of benchmark models $\left(1.35 \mathrm{M}_{\odot}, 1.65 \mathrm{M}_{\odot}, 1.95 \mathrm{M}_{\odot}\right)$ 

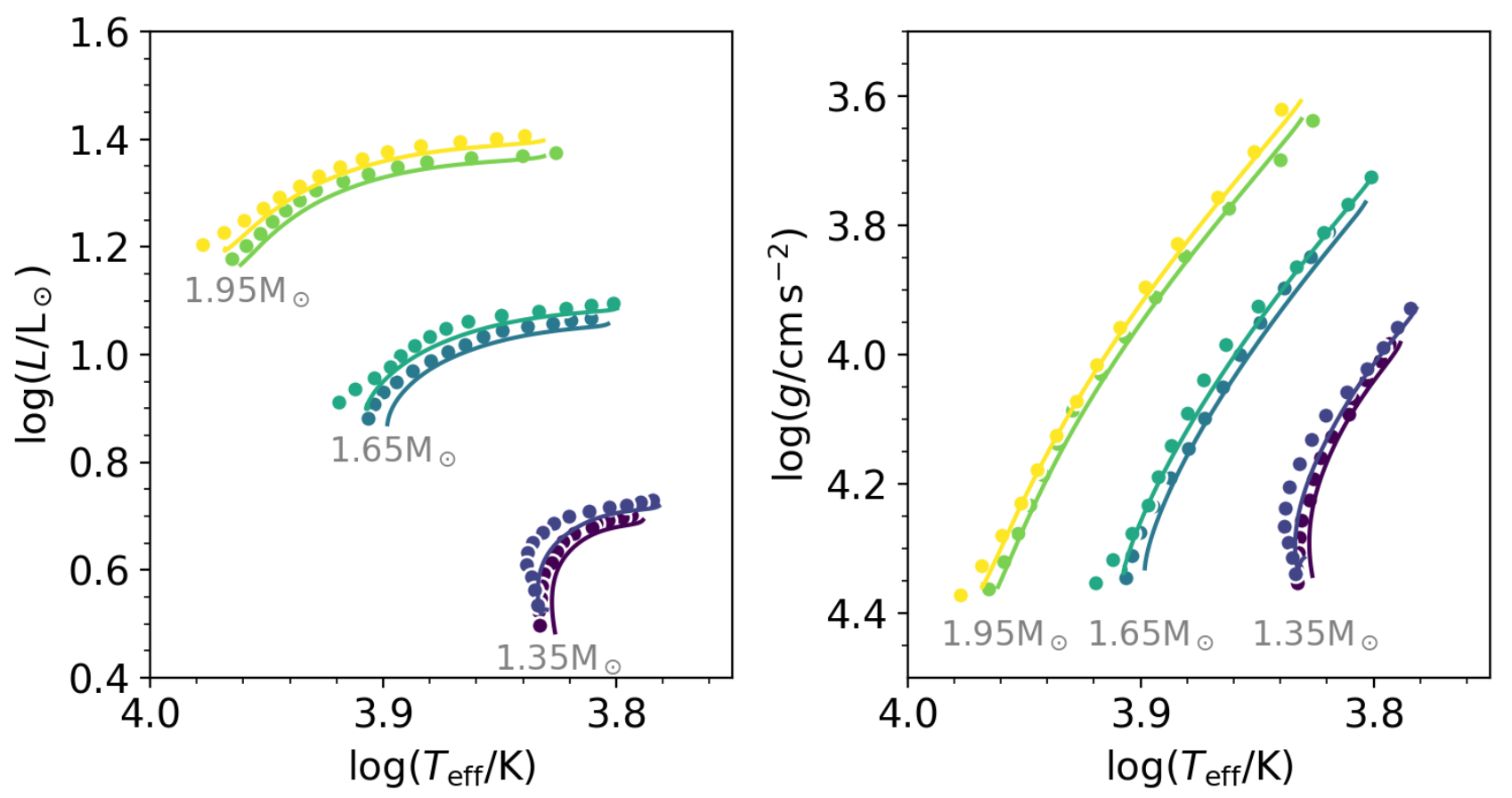

Fig. 5. Comparison between the predictions of C-3PO (dots) and the MESA benchmark evolution tracks (solid lines) for $Z=0.012$ and $Z=0.023$. The predictions are sampled from $X_{\mathrm{c}}=0.70$ to 0.05 with a step size of 0.05 .

which were not included in the training/validation set. We use radial orders $n \in[20,60]$, and the photospheric observables from the MESA model as input, where we assume the following uncertainties: $\sigma_{\log L / \mathrm{L}_{\odot}}=0.05 \mathrm{dex}, \sigma_{\log T_{\text {eff }}}=0.015 \mathrm{dex}$, and $\sigma_{\log g}=0.6$ dex. The metallicity and rotation frequency are fixed to the values of the models in the fit, since these are also known for the observations. Two examples are shown in Figure 6 for a young star and an old star. The accuracy of the NN is less for patterns with clear mode trapping, yet in both cases the periods are still predicted with enough accuracy such that, with the inclusion of the photospheric observables, $M_{\star}$ and $X_{\mathrm{c}}$ can be recovered. In Figure 7, we show the mass and $X_{\mathrm{c}}$ values of all the inputs models, and the obtained estimates from C-3P0. On average, the input mass, $X_{\mathrm{c}}$, and $f_{\mathrm{ov}}$ are recovered within $2 \%, 15 \%$, and $16 \%$, respectively. It should also be noted that, as mentioned before, degeneracies exist between young lower-mass stars and old higher-mass stars. Thus, even if the NN would be able to perfectly predict the pulsation periods, exactly recovering a (perturbed) input model would still be impossible (see middle panels of Figure 6).

\subsection{Observational benchmark}

One of the stars in our sample, KIC 9751996, has been analysed by Mombarg et al. (2020) who modelled the star using models both with and without atomic diffusion (including radiative levitation). As mentioned before, in this paper we use the same input physics in our models compared to their grid without atomic diffusion. It should be noted, however, that in this work only the periods of the prograde mode are modelled, whereas in the work by Mombarg et al. (2020) the periods of the retrograde, zonal, and prograde are modelled simultaneously.

This yields $M_{\star}=1.740_{-0.169}^{+0.009} \mathrm{M}_{\odot}, X_{\mathrm{c}}=0.241_{-0.040}^{+0.147}$, and $f_{\mathrm{ov}}=$ $0.0124_{-0.0024}^{+0.0121}$, all of which are consistent with the values found by Mombarg et al. (2020). The predicted period spacing pattern of this best-fitting model from $\mathrm{C}-3 \mathrm{PO}$ is shown in Figure 8.

\section{Application to the sample}

For all 37 stars in our sample, we apply the modelling scheme discussed in Section 3 to constrain $M_{\star}, X_{\mathrm{c}}$, and $f_{\mathrm{ov}}$, in order to compare the results with those based on $\Pi_{0}$ obtained by Mombarg et al. (2019). We recall that our aim is not to achieve good fits to the dips in the patterns, as this requires stratified envelope mixing profiles (Pedersen et al. 2021). Here, we merely wish to compare the capacity of the NN with previous modelling based on actual equilibrium models rather than a numerical NN approximation thereof.

Figure 9 shows the mass and $X_{\mathrm{c}}$ of the best model predicted by $\mathrm{C}-3 \mathrm{PO}$ of each star. As is expected from the position of the $\gamma$ Dor instability strip (Dupret et al. 2004), there is a correlation between the estimated mass and age, i.e., no young, more massive or old, less massive stars are observed, as already found by Mombarg et al. (2019). KIC 2710594, KIC 3448365, KIC 6678174, KIC 6953103, KIC 7380501, KIC 8645874, KIC 11099031, and KIC 11294808 are stars for which a particularly complicated period spacing pattern is observed, i.e., large deviations in the observed morphology occur, compared to what we expect from the pulsation models limited to the physics described in this work. For these stars, part of the pattern has not been taken into account in the fitting. In the case of KIC 5522154, KIC 5708550, KIC 6678174, KIC 6953103, KIC 7365537, the best solutions to the observed mode periods do not seem to be in agreement with the photospheric observables. The former and latter are fast rotators $\left(f_{\text {rot }}>2.15 \mathrm{~d}^{-1}\right.$, Van Reeth et al. (2016)), and hence the inferred $T_{\text {eff }}$ could suffer a larger systemic error due to the larger uncertainty of the spectrum normalization and gravity darkening. For the other three stars, it is primarily the luminosity which does not agree with the observed pulsations. In any case, for these five 


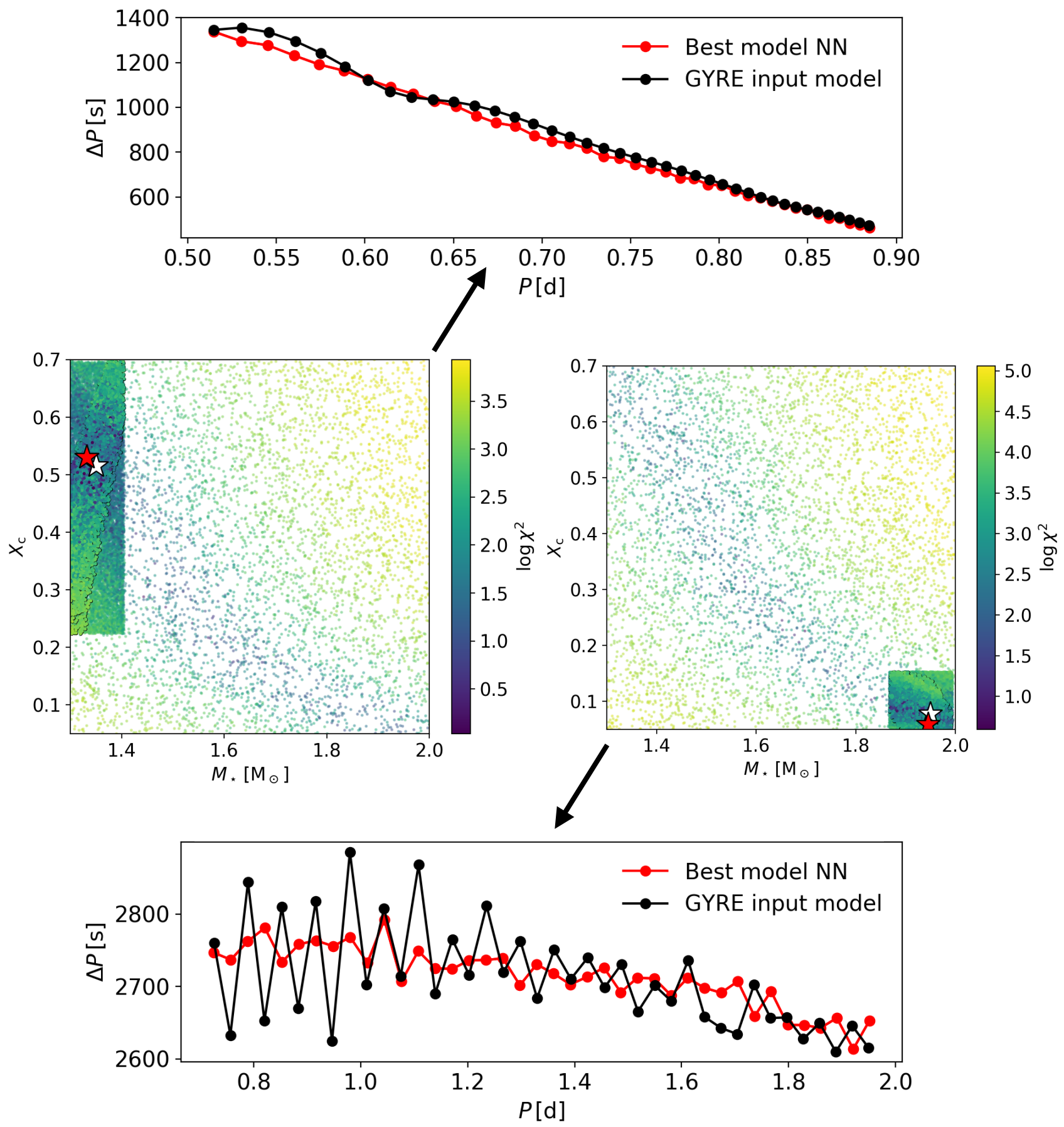

Fig. 6. Examples of the recovered mass and $X_{\mathrm{c}}$ of two benchmark models (white stars: input, red stars: best model predicted by NN). Although the period spacings of trapped modes are more difficult to predict (bottom panel), the inclusion of the photospheric observables yields accurate predictions of the mass and $X_{\mathrm{c}}$. The middle panels show the sampling density is increased in the parameter space where the predicted photospheric observables comply with those of the input model, as explained in Section 3. Model 1: $\left(M_{\star}, X_{\mathrm{c}}, Z, f_{\mathrm{ov}}, D_{0}, f_{\mathrm{rot}}\right)=$ $\left(1.35 \mathrm{M}_{\odot}, 0.515,0.014,0.0225,1 \mathrm{~cm}^{2} \mathrm{~s}^{-1}, 0.7457 \mathrm{~d}^{-1}\right)$. Model 2: $\left(M_{\star}, X_{\mathrm{c}}, Z, f_{\mathrm{ov}}, D_{0}, f_{\mathrm{rot}}\right)=\left(1.95 \mathrm{M}_{\odot}, 0.08,0.014,0.0225,1 \mathrm{~cm}^{2} \mathrm{~s}^{-1}, 0.0449 \mathrm{~d}^{-1}\right)$. Typical uncertainties on $\Delta P$ are of the order of several tens of seconds.

stars, no $2-\sigma$ cutoff in $L, T_{\text {eff }}$, and $\log g$ is imposed, resulting in a larger uncertainty on $M_{\star}$ and $X_{\mathrm{c}}$, as can be seen in Figure 9.

We have investigated the effect of adding the rotation frequency (within the observational uncertainties) as an additional free parameter in the fitting on the MLEs. For both the fastest rotating star (KIC 12066947) and the star with the least precisely determined rotation rate (KIC 6678174), fixing the rotation rate in the modelling yields consistent estimates of the varied stellar parameters, compared to when we allow it to vary.

In addition to $(\ell, m)=(1,1)$ modes, $(\ell, m)=(1,0)$ modes for KIC 4846809 and KIC 9595743, and an $(\ell, m)=(2,2)$ mode for KIC 11294808 have been observed by Van Reeth et al. (2016). 


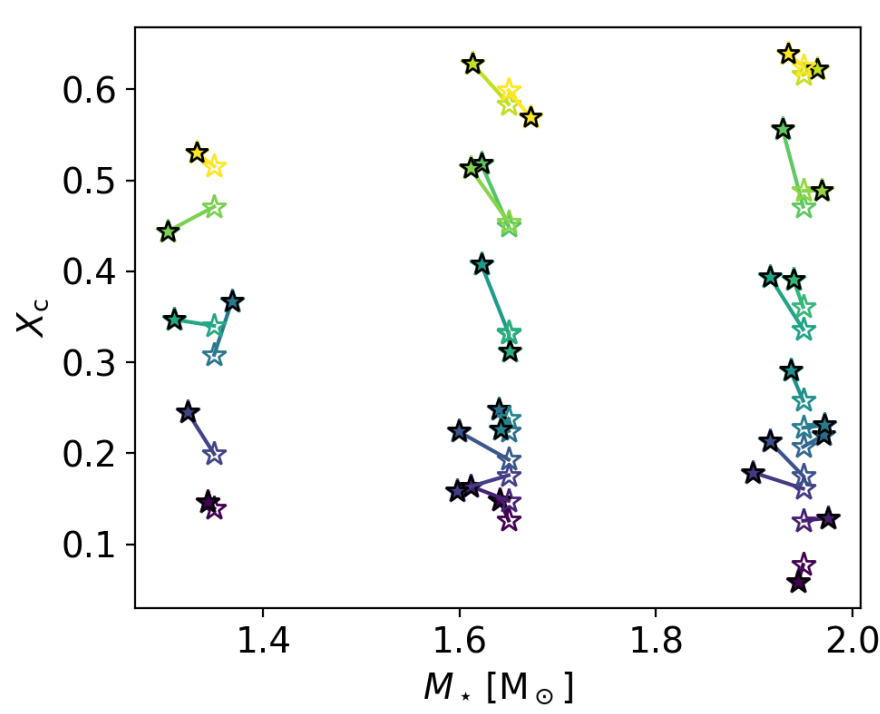

Fig. 7. Mass and $X_{\mathrm{c}}$ values of the benchmark models (open symbols) and the corresponding retrieved values from the best-fitting model computed with C-3P0 (filled symbols). For the benchmark models $Z=$ 0.014 and $f_{\text {ov }} \in[0.0175,0.0225]$.

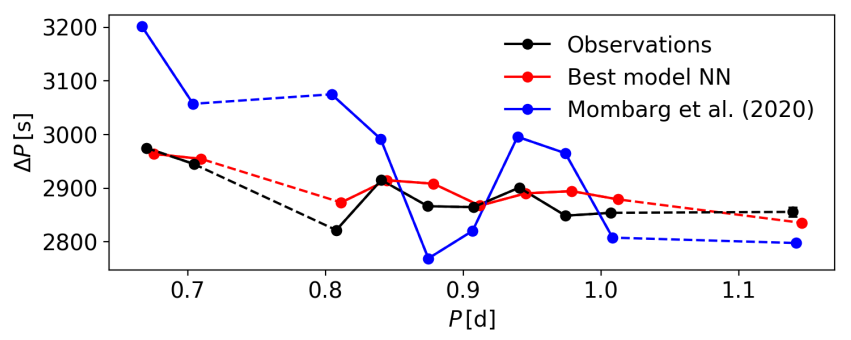

Fig. 8. Observed period spacing pattern of KIC 9751996 from Van Reeth et al. (2016) (black dots, prograde dipole mode only) and the best-fitting pattern predicted with C-3P0 (red dots). For comparison, the solution from Mombarg et al. (2020) without atomic diffusion (their model M01) is also shown (blue dots). The observational uncertainties are typically smaller than the symbol size.

The additional period spacing patterns of these three stars have also been taken into account in Step 5 of the modelling scheme (Section 3).

Table F.3 lists the parameters of the best models found by C-3PO and of the best-fitting GYRE model. In Appendix D, we show the theoretically predicted period spacing patterns for all stars in our sample, as well as the distribution of radial orders, similar to the result from Li et al. (2020). Moreover, the BruntVäisälä frequency profiles of the best-fitting models are shown in Fig. D.39. In many cases, we are not able to reproduce the wiggly characteristics of the observed patterns, indicating that the physics used in this work is still incomplete and requires improvement. Therefore, these deviations between the patterns from our best solution and the observed ones offer a fruitful guide to future studies with the aim to explain the morphology of the patterns, by means of different core-boundary mixing prescriptions and/or introducing stratified envelope mixing profiles. In order to guide such future studies, we focus on one of the stars in our sample which shows clear 'wiggles' in its observed period spacing patterns, i.e., KIC 11294808. With the overshoot prescription used in this work (Eq. 1), such wiggles are only observed in our models at a level of $D_{0}=0.05 \mathrm{~cm}^{2} \mathrm{~s}^{-1}$ (see Fig. E.1). The same conclusion is found for this star when we recompute the MESA and GYRE models, but this time using a steplike penetrative convection prescription as convective-boundary mixing prescription, where we explore $\alpha_{\text {ov }} \in[0.05,0.35 ; 0.05]$ (other parameter ranges kept the same). We obtain the same best model as for an exponential core overshoot in terms of mass and evolutionary stage. This corresponding best convective penetration model has a value of $\alpha_{\mathrm{ov}}$ that is ten times the value of $f_{\text {ov }}$, in agreement with previous studies where such comparisons were made (Moravveji et al. 2016). This solution is also shown in Fig. D in the Appendix (grey open circles).

The remaining differences between the observed and predicted mode periods via the NN seen in most of the stars is due to the lack of stratified envelope mixing. As shown by Pedersen et al. (2021), the latter results in structure to occur in the patterns. Such structure can have various physical causes, for example, atomic diffusion with radiative levitation (e.g. Deal et al. 2020; Mombarg et al. 2020), magnetism (e.g. Prat et al. 2019; Van Beeck et al. 2020, which causes saw-tooth like features), shear instabilities due to rotation or wave mixing (Pedersen et al. 2021), or nonlinear effects (discussed later on). Our results open the way to include those types of phenomena by modelling the residuals between the periods predicted by the current initial NN and the observed ones.

As an extra sanity check to assess the quality of the NN solution, we show in Figure 10 the $\Pi_{0}$ value derived from our best model and compare it to the observational values from Van Reeth et al. $(2016,2018)$ as derived from the mode identification along with the near-core rotation frequency. In general, we find adequate correspondence between the two ways of estimating $\Pi_{0}$. The most significant discrepancy is found in KIC 11099031 (data point indicated in red). This is one of stars for which Christophe et al. (2018) found a much lower value of $\Pi_{0}$, compared to Van Reeth et al. (2016) (see Fig. 5 in Ouazzani et al. (2019)).

\subsection{On the possible origins of $C B M$}

The parameters of the best-fitting models discussed in this section are those extracted from the MESA and GYRE models, computed in Step 5 of the modelling scheme. As illustrated in Figure 11, the modelling of individual pulsations instead of the method employed by Mombarg et al. (2019) allows for a better constraint on $f_{\mathrm{ov}}$, as the individual mode periods lead to less degeneracy with respect to the stellar mass and age. In addition, the luminosity is in general more precisely determined than $\log g$ for F-type stars, which further reduces the degeneracy between the stellar mass and age. The overshoot parameter has been asteroseismically calibrated for eight solar-like oscillators with a convective core by Deheuvels et al. (2016), covering a mass range from roughly 1.1 to $1.45 \mathrm{M}_{\odot}$. They observe an increase of the overshoot parameter with increasing mass, although this trend is much less outspoken for $M_{\star}>1.25 \mathrm{M}_{\odot}$. The results from Deheuvels et al. (2016) for models with microscopic diffusion are shown in Figure 11 in grey. To convert from a step-like overshoot (penetrative, $\alpha_{\text {ov }}$ ) to the exponential-like overshoot (diffusive) parameterizaton used in this work, we have used the approximation $\alpha_{\mathrm{ov}} \approx 10 f_{\mathrm{ov}}$ as inferred by Claret \& Torres (2017). A similar correlation between overshooting and the mass was found by these authors from isochrone fitting of binary systems, plateauing to a value of $\sim 0.0175$ for $M_{\star} \gtrsim 2.0 \mathrm{M}_{\odot}$.

Yet for the single stars modelled in this work, we do not observe such a mass-overshoot relation, albeit a much smaller range in mass is probed. Furthermore, for a significant portion of the sample, we obtain higher values for $f_{\mathrm{ov}}$ than those from 

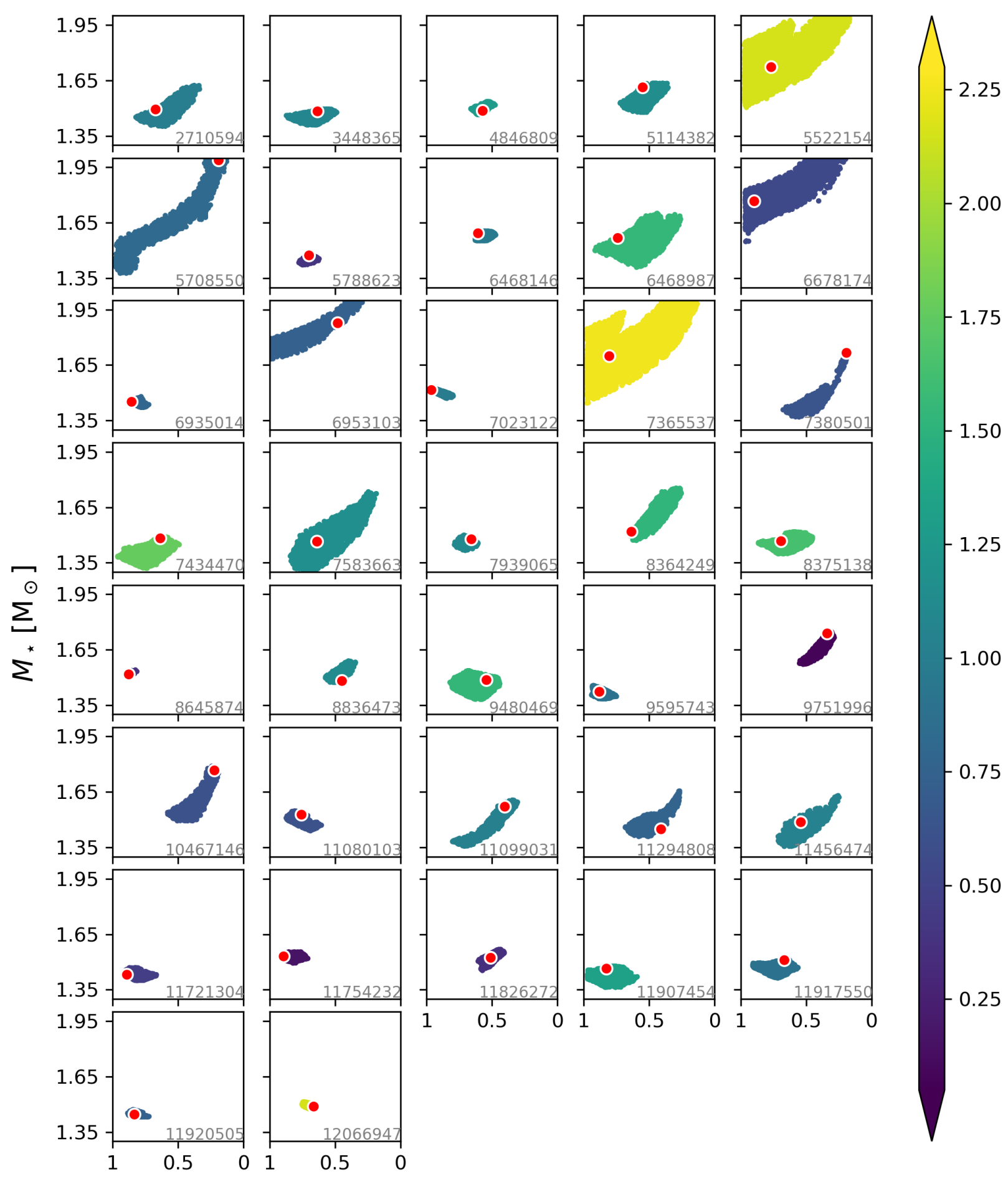

2.00
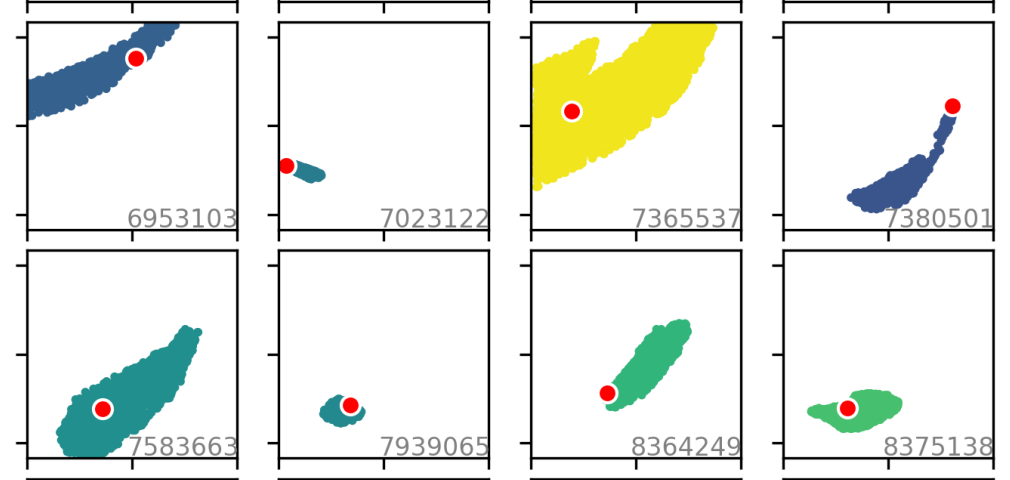

$-1.75$
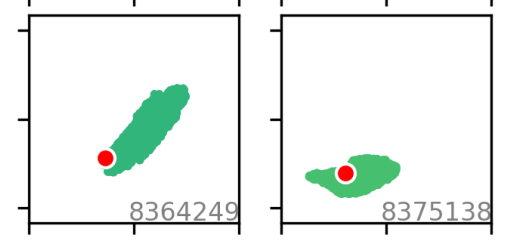

$-1.50$
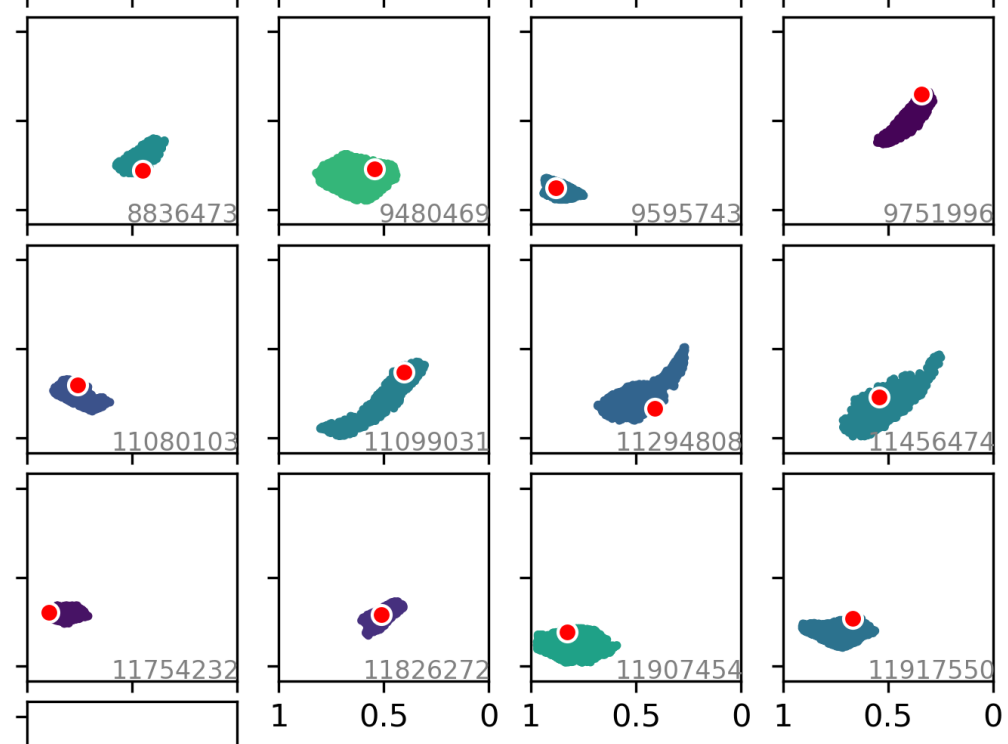

0.50

0.75

1.25

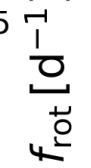

$-1.00$
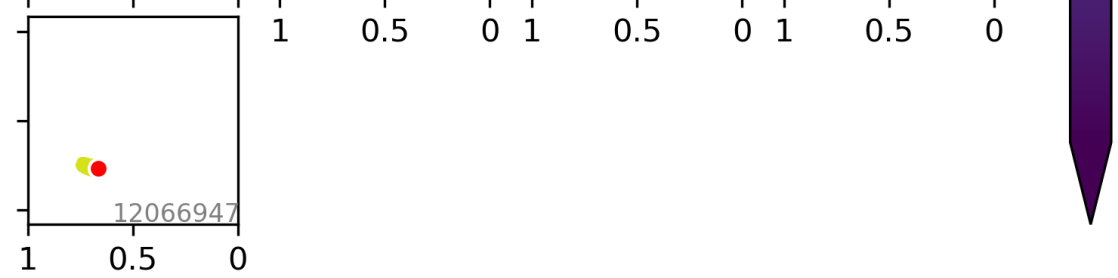

0.25

$$
X_{\mathrm{c}} / X_{\mathrm{ini}}
$$

Fig. 9. Correlation structure of the $68 \%$-confidence intervals of $M_{\star}$ and $X_{\mathrm{c}} / X_{\text {ini }}$ for all 37 stars in our sample from modelling the dipole period spacing patterns with C-3PO. The MLE is indicated by the red dot. The KIC number is indicated in the bottom right corner of each plot.

Claret \& Torres (2017). Caution is advised when comparing overshoot values between different studies, as the exact location in the star where the overshoot (core-boundary mixing; CBM) profile starts is set by an additional parameter, $f_{0}$, normalized by the local pressure scale height, for which we use 0.005 in this work. In Figure 12, the extent of the overshoot zone is plotted against $X_{\mathrm{c}} / X_{\mathrm{ini}}$. The size of the overshoot region is defined as the difference between $r_{\mathrm{ov}}$ and $r_{\mathrm{cc}}$, where $r_{\mathrm{cc}}$ is defined as the radius where the temperature gradient transitions from adiabatic to radiative, and $r_{\mathrm{ov}}$ is the radius where the overshoot zone ends 


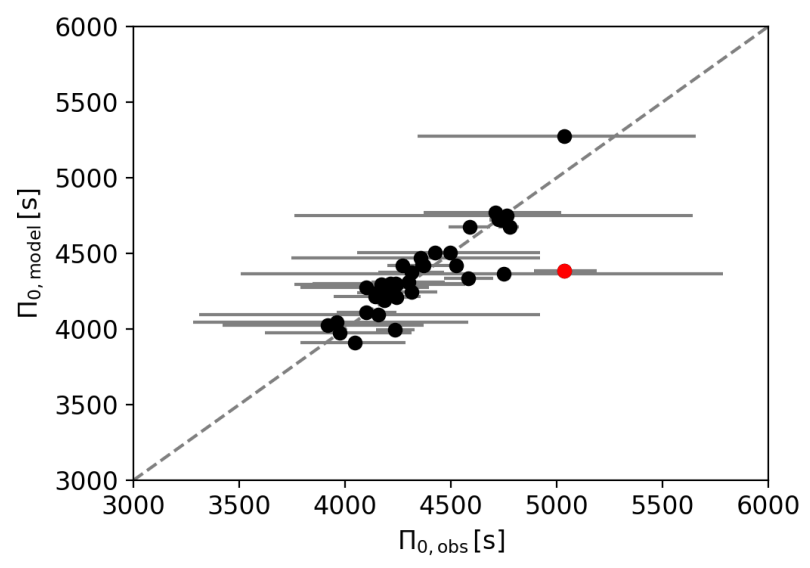

Fig. 10. Comparison between the $\Pi_{0}$ values derived by Van Reeth et al. $(2016,2018)\left(\Pi_{0, \text { obs }}\right.$, simultaneously with $\left.f_{\text {rot }}\right)$ versus the values found from the best models presented in Table F.3 of this paper $\left(\Pi_{0, \text { model }}\right)$. The grey dashed line indicates perfect correspondence. The outlier KIC 11099031 is indicated in red.

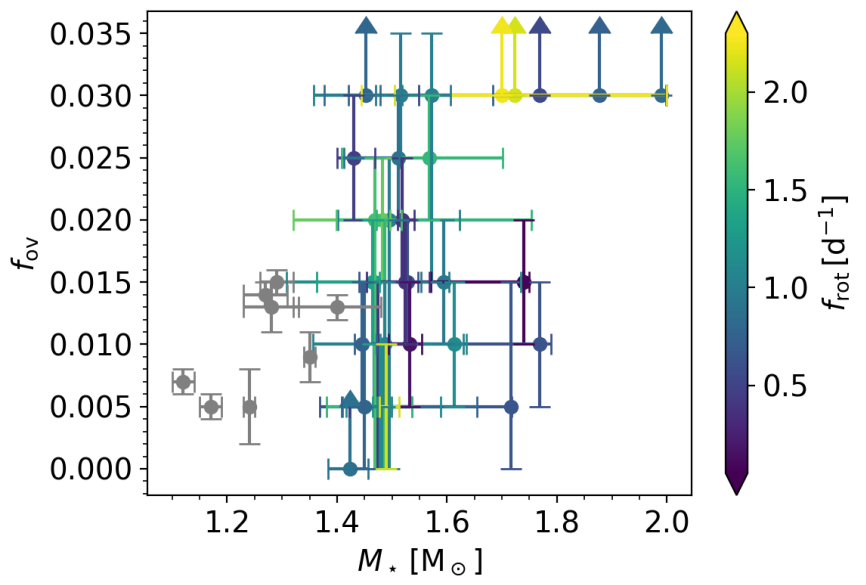

Fig. 11. Derived masses and overshoot parameters for all stars in our sample from GYRE models. For comparison, inferred overshoot parameters of low-mass stars by Deheuvels et al. (2016) (solutions with microscopic diffusion, where we have used $\alpha_{\mathrm{ov}} \approx 10 f_{\mathrm{ov}}$ ) are plotted in grey.

(cf. Figure 1). No evident correlation is observed between the extent of the overshoot zone and the evolutionary stage, although the most evolved stars in our sample typically have smaller overshoot zones. It should be noted that if the value of $f_{\text {ov }}$ is dependent on the stellar age, this is not taken into account in current SSE models. The modelling of period spacing patterns does not probe the value of $f_{\mathrm{ov}}$ at the current age of the star, but rather the constant value that is needed to have the correct core properties at the age when the observations were taken. As illustrated in Figure 13, we observe a correlation between the core mass, $m_{\mathrm{cc}}$, and the extent of the overshoot region. An increase in $r_{\mathrm{ov}}-r_{\mathrm{cc}}$ with increasing $m_{\mathrm{cc}}$ is observed, where there is an offset between younger and older stars. For the less massive stars, the convective core grows in mass at first, whilst for the more massive stars, the core recedes throughout the MS (cf. Figure 2 of Mombarg et al. 2019). This suggests that the correlation between $r_{\mathrm{ov}}-r_{\mathrm{cc}}$ and $m_{\mathrm{cc}}$ is dependent on the core density. The fractional core mass versus the stellar mass is shown in Figure 14.

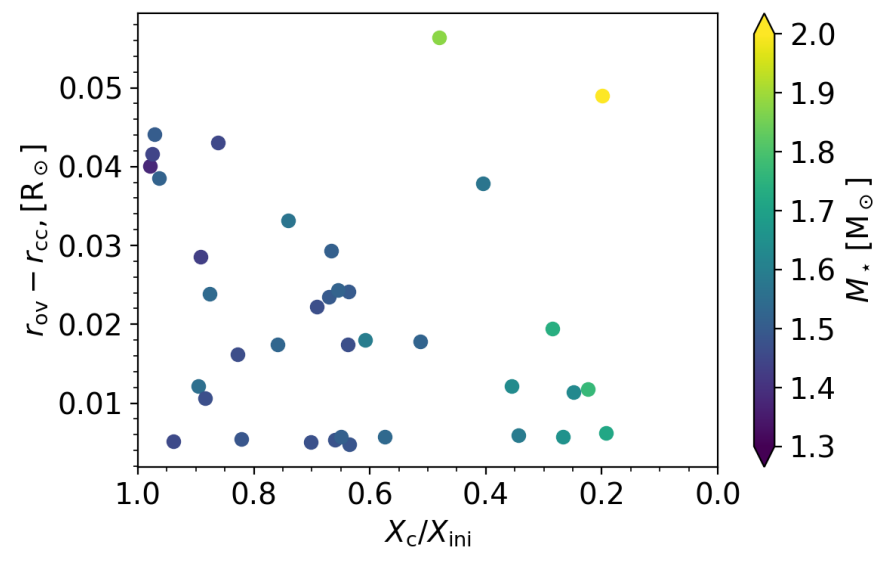

Fig. 12. Extent of the overshoot zone plotted against $X_{\mathrm{c}} / X_{\mathrm{ini}}$.

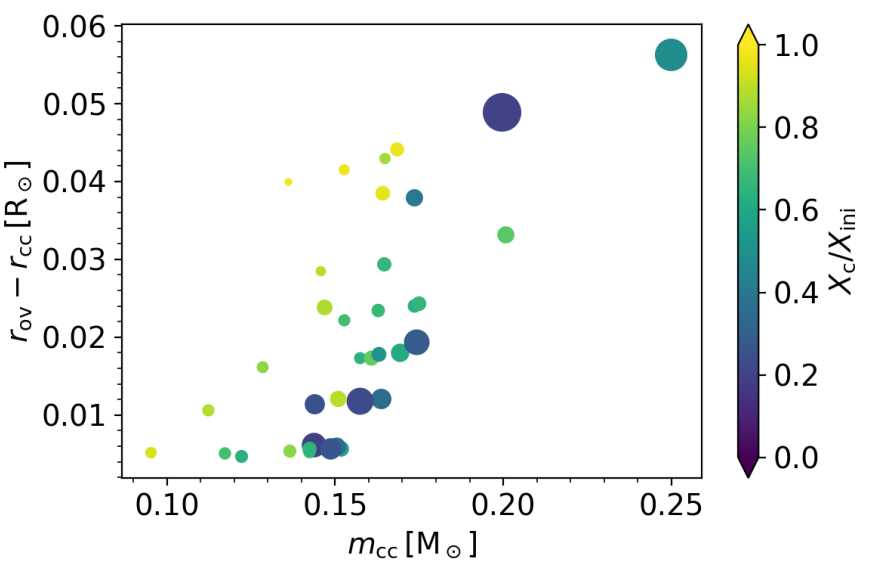

Fig. 13. Correlation between the convective core mass and extent of the overshoot zone. The symbol size is indicative of the mass.

Although we refer to $f_{\text {ov }}$ as the convective core overshoot parameter, $f_{\text {ov }}$ encompasses all forms of mixing occurring close to the core boundary. If $\mathrm{CMB}$ is induced via rotational shears between the core and the envelope, a dependence between the amount of CMB and the rotation rate is expected. In Figure 15 the inferred extent of the overshoot zone, relative to the core size is plotted as a function of the near-core rotation rate from Van Reeth et al. (2016) $\left(\Omega=2 \pi f_{\text {rot }}\right)$, scaled by $\sqrt{G m_{\mathrm{cc}} / r_{\mathrm{cc}}^{3}}$, where $G$ is the gravitational constant ${ }^{1}$. No evidence is found that the $\mathrm{CMB}$ increases with a faster rotating near-core region. To fully rule out the connection between rotation and the extent of the overshoot zone, the rotation of the convective core itself needs to be measured. Saio et al. (2021) have derived the rotation of the convective core in 16 rapidly rotating $\gamma$ Dor stars from the sample of Li et al. (2020) by studying the coupling between inertial modes in the convective core and $\mathrm{g}$ modes in the radiative envelope. These authors found only small differences with the rotation rates derived from $g$ modes in the TAR framework for the majority of stars.

1 No scaling of $\Omega$ does not reveal any correlation either. 


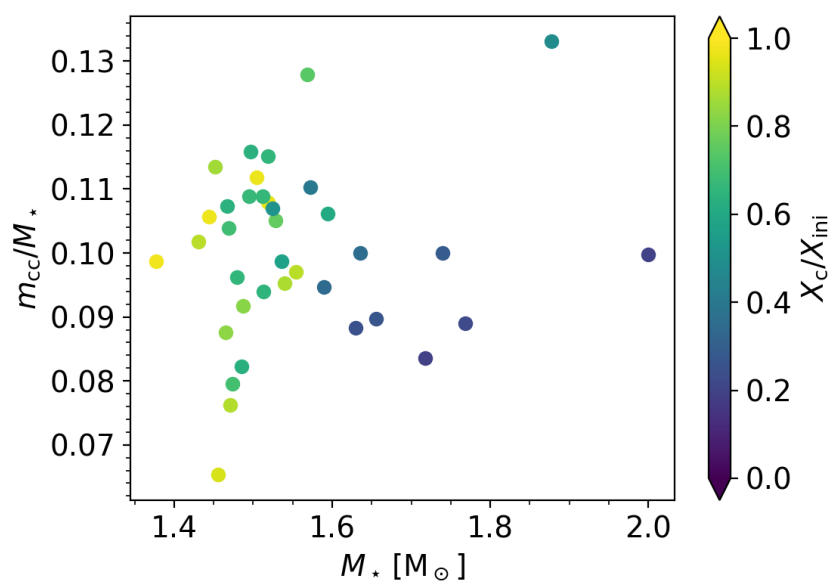

Fig. 14. Derived fractional convective core mass versus stellar mass.

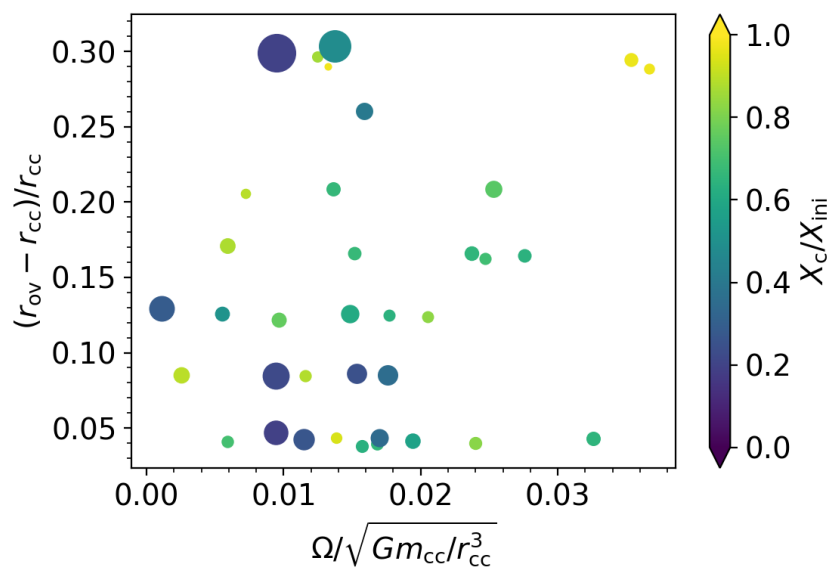

Fig. 15. Extent of the overshoot region with respect to the radius of the convective core as a function of the angular rotation rates from Van Reeth et al. (2016), scaled with frequency $\sqrt{G m_{\mathrm{cc}} / r_{\mathrm{cc}}^{3}}$, where $m_{\mathrm{cc}}$ and $r_{\mathrm{cc}}$ are the core mass and radius, respectively. The symbol size is indicative of the stellar mass.

\subsection{Angular momentum of the sample}

The AM of a star, $J$, is defined as

$J=\int r^{2} \Omega(r) \mathrm{d} m$

For eight stars in our sample, Van Reeth et al. (2018) have measured the ratio between the near-core rotation rate and the surface rotation rate, suggesting all eight stars are quasi-rigidly rotating. In addition, Saio et al. (2021) found the $\gamma$ Dor stars in their sample to be rotating nearly uniformly as well. Therefore, assuming $\Omega(r)$ is constant throughout the star and that typical mass loss in F-type stars $\left(\dot{M} \simeq 10^{-13} \mathrm{M}_{\odot} / \mathrm{yr}\right)$ is too small to carry away significant amounts of AM from the star, we can infer the rotation rate at any point in time, since $J$ is constant. Figure 16 shows the fraction of AM in the convective core, compared to the total AM of the whole star, $J$, as a function of stellar age, $\tau$. Furthermore, two examples of the evolution of the AM of the convective core ${ }^{2}, J_{\text {cc }} / J$ for KIC $2710594\left(\sim 1.5 \mathrm{M}_{\odot}\right)$

\footnotetext{
$2 J_{\mathrm{cc}}$ is computed by discretizing the integral in Eq. (10), i.e. $J_{\mathrm{cc}}=$ $\sum_{i} r_{i}^{2} \mathrm{~d} m_{i}$, where $i$ runs over the cells in the model below the convective core, and $r$ and $\mathrm{d} m$ are the central radius and enclosed mass of a cell, respectively.
}

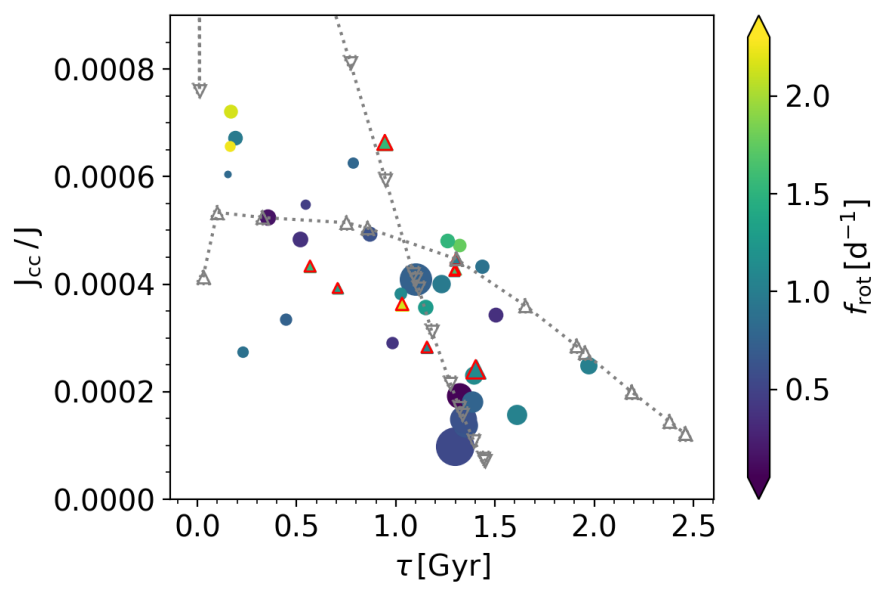

Fig. 16. Fractional AM of the convective core to total AM, assuming rigid rotation throughout the MS. Stars with detected Rossby modes are plotted as triangles and highlighted in red. The symbol size is indicative of the mass. The grey symbols indicate the predicted evolution of $J_{\text {cc }} / J$ (assuming rigid rotation) for KIC 2710594 (upward triangles) and KIC 6953103 (downward triangles).

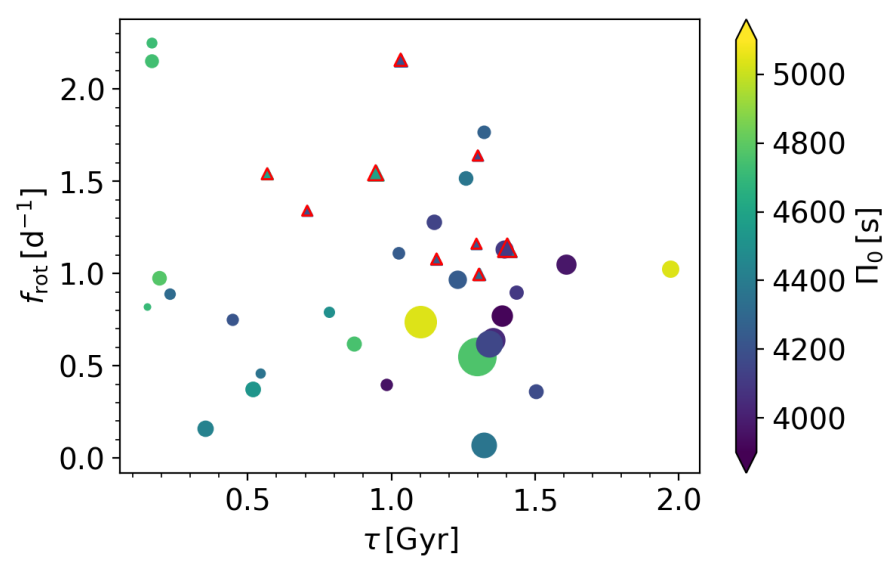

Fig. 17. Near-core rotation rates (and $\Pi_{0}$ ) from Van Reeth et al. (2016, 2018 ) as a function of age $\tau$ (this work). Stars with detected Rossby modes are highlighted as red triangles. The symbol size is indicative of the mass.

and $\mathrm{KIC} 7434470\left(\sim 1.9 \mathrm{M}_{\odot}\right)$ when rigid rotation is assumed, are shown.

Mombarg et al. (2019) observed that stars with detected Rossby modes are situated across the entire MS (based on $\left.X_{\mathrm{c}} / X_{\text {ini }}\right)$, which is in line with our findings. In Figure 17, we show the near-core rotation as a function of absolute age, $\tau$, and highlighted stars in red for which Van Reeth et al. (2016) detected Rossby modes. Again, we do not find any correlation between a star's age and the presence of Rossby modes.

When rigid rotation is assumed, the initial rotation frequency at the start of the MS can be estimated. Figure 18 shows the distribution of the rotation frequencies near ZAMS (i.e. not more than $3 \%$ of the initial hydrogen in the core burnt) as well as the distribution of the present rotation frequency (Van Reeth et al. 2016,2018 ). While the distribution of the present-day near-core rotation frequencies corresponds to the one found by $\mathrm{Li}$ et al. (2020), we find a broader distribution for the near-ZAMS rotation rates, which peaks around $1.8 \mathrm{~d}^{-1}$. 


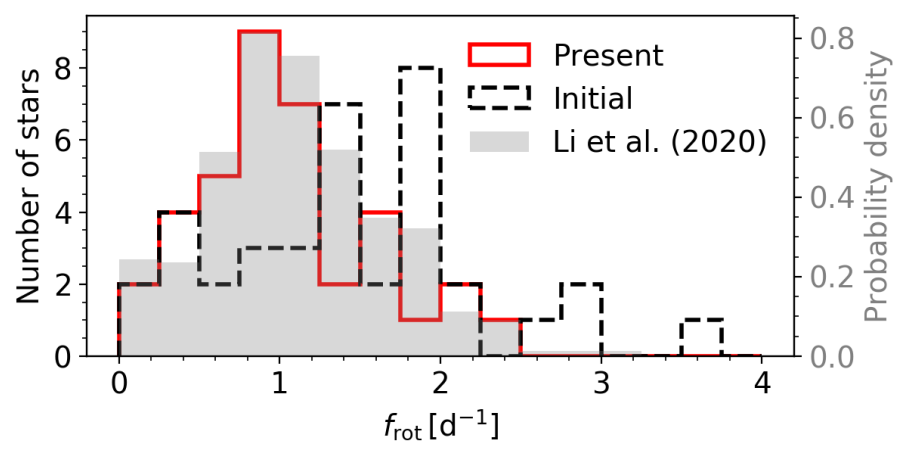

Fig. 18. Red: Distribution of the present near-core rotation frequencies from Van Reeth et al. (2016, 2018). Black: Distribution of the rotation rate near the ZAMS, assuming rigid rotation throughout the MS. Grey: Normalized distribution from Li et al. (2020).

\subsection{Mode interaction in KIC 12066947}

One particularly interesting star in our sample is KIC 12066947. It shows just one characteristic dip in the period spacing pattern at 0.38 days. Such a single dip is not expected to be caused by a chemical gradient because this introduces multiple dips (Miglio et al. 2008). Recently, Saio et al. (2021) were able to reproduce the sharp dip observed in the period spacing pattern of this star, using the formalism by Lee \& Baraffe (1995) to compute the coupling between $g$ modes in the radiative envelope and an inertial mode in the convective core. To exclude the scenario of constant envelope mixing combined with a chemical gradient in the core boundary layer as a possible second explanation, we again repeat Step 5 (Section 3), but with extremely inefficient envelope mixing, that is, $D_{0}=0.05 \mathrm{~cm}^{2} \mathrm{~s}^{-1}$. As can be seen from the best pulsation model selected among those computed from the best stellar models according to C-3P0, see Figure 19, a low $D_{0}$ does introduce a dip in the pattern around the observed dip in period spacing close to 0.38 days. However, the additional theoretically predicted dips are not observed. A rotation rate of $25_{-0.10}^{+0.09} \mu \mathrm{Hz}$ for KIC 12066947 has been measured by Van Reeth et al. (2016). Assuming a uniform rotation profile at $25 \mu \mathrm{Hz}$, Ouazzani et al. (2020) have computed the radial order at which the interaction between inertial and gravito-inertial modes occurs. According to the parameters derived for KIC 12066947 in this work, the star is most akin to the mid-MS model from Ouazzani et al. (2020), for which the mode interaction is estimated to occur at $n_{\mathrm{pg}}=-44$. The dip in the period spacing pattern occurs at $n_{\mathrm{pg}}=-44$, therefore, if the dip is caused by interaction between gravito-inertial and pure inertial modes, it should occur close to the dip. Hence, the observed dip in the period spacing pattern of KIC 12066947 is most likely caused by mode interaction, confirming the results by Ouazzani et al. (2020) and Saio et al. (2021).

\subsection{Comparison with Mombarg et al. (2019)}

In Figure 20, we compare the MLE for $M_{\star}, X_{\mathrm{c}}$, and $f_{\mathrm{ov}}$ from C-3PO (this work) with those from Mombarg et al. (2019). Several key differences between the NN methodology in this work and theirs should be noted while evaluating the comparison, as summarised in Table 4. Keeping these differences in mind, the agreement among the estimated parameters is adequate, given the uncertainties in the parameter estimation. This is not the case for all stars in terms of their masses, however. The mass estimates from the NN methodology are in general lower compared to those of Mombarg et al. (2019). This is not a consequence

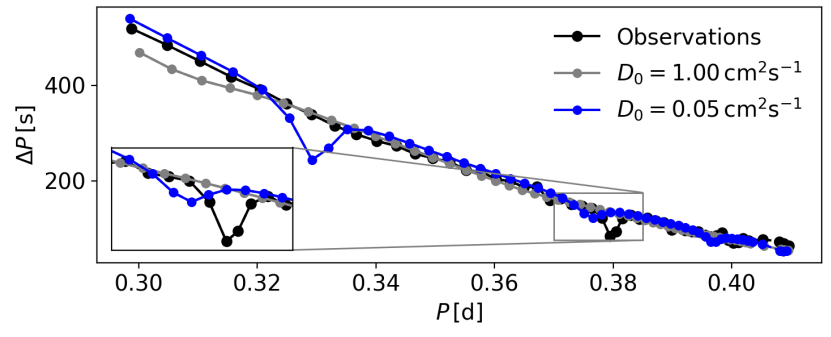

Fig. 19. Best-fitting model with $D_{0}=0.05 \mathrm{~cm}^{2} \mathrm{~s}^{-1}$ to the observed period spacing pattern of KIC 12066947 (black dots, Van Reeth et al. 2016). For reference the best-fitting with the standard $D_{0}=1.0 \mathrm{~cm}^{2} \mathrm{~s}^{-1}$ used in this work is shown in grey.

of the more complex NN modelling strategy adopted here, however, but rather due to the inclusion of the stellar luminosities calculated from the Gaia DR2 data as a constraint imposed in the modelling. Figure F.1 in the appendix shows the effect of including the Gaia luminosity as an extra constraint in the modelling on the inferred stellar mass. A systematic underestimation of the stellar masses deduced from Gaia DR2 data was also found by Pedersen et al. (2021) in their asteroseismic modelling of B-type stars. We conclude that the NN itself performs well as an asteroseismic modelling strategy and opens the way forward for applications to large samples of $\gamma$ Dor pulsators and for the use of more complex stellar models, including parametrized stratified envelope mixing profiles.

\section{Conclusion \& Discussion}

In this paper, we have constructed a forward modelling scheme for gravito-inertial modes in $\gamma$ Dor stars, and used it to estimate the stellar mass, the central hydrogen mass fraction, and the overshooting parameter. We have eliminated the need of large grids of stellar models by training a dense neural network on a coarse grid of stellar evolution and pulsation models to predict the corresponding oscillation periods $(n \in[15,91])$ and the luminosity, effective temperature, and surface gravity, given the mass, hydrogen mass fraction in the core, metallicity, coreboundary mixing and radiative envelope mixing efficiency, and rotation rate. All of the input parameters of the network have been varied within appropriate ranges of $\gamma$ Dor stars, making it a versatile tool for estimating stellar parameter ranges with minimal computational effort. The C-3PO neural network, comprising of eight different subnetworks has been applied to a sample of $37 \gamma$ Dor stars for which period spacing patterns have been detected by Van Reeth et al. (2016). For $T_{\text {eff }}$ and $\log g$, we relied on the measurements from Van Reeth et al. (2015b) and have derived luminosities for the sample, using Gaia DR2 distances from Bailer-Jones et al. (2018).

Using the mass and $X_{\mathrm{c}}$ estimates of C-3PO, we have computed small grids of stellar pulsation models for each star, as to further constrain the overshoot $f_{\mathrm{ov}}$ as well. We find no evidence for the core boundary mixing efficiency to correlate with stellar mass, age, or rotation rate. However, we do observe stars with larger core masses tend to have larger overshoot regions. Furthermore, we find that Rossby modes are only detected in the more evolved stars in our sample, which is only seen when the actual stellar age is used instead of $X_{\mathrm{c}} / X_{\text {ini }}$. Yet, a much larger sample is needed to conclude whether Rossby modes are indeed not observed for stars younger than about 1 Gyr. Li et al. (2020) compiled a sample of $611 \gamma$ Dor stars, 83 of which have observed 

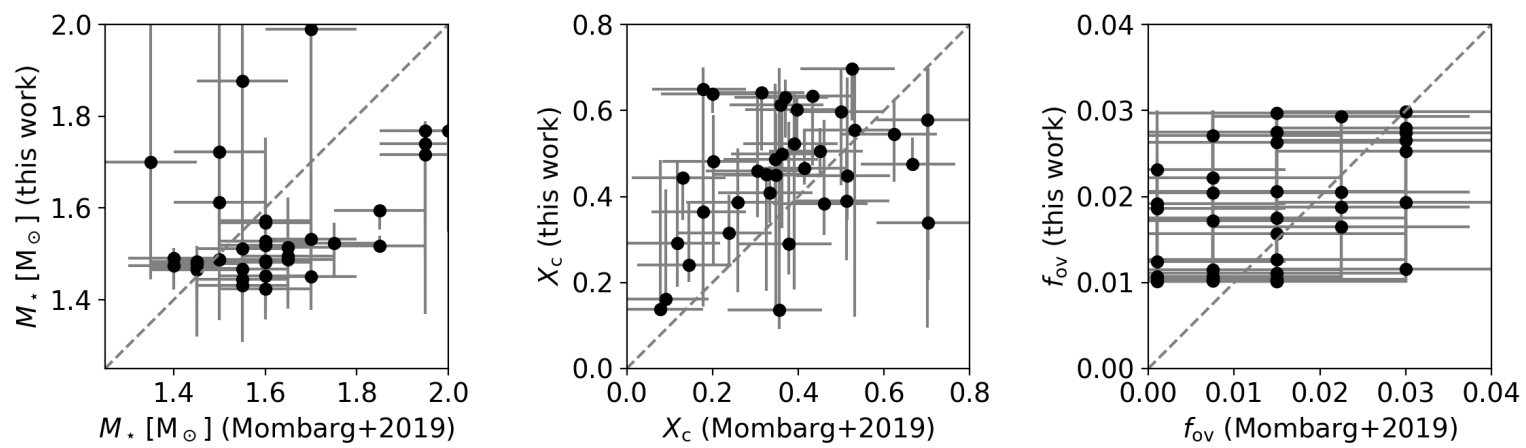

Fig. 20. Predictions of the C-3PO NN versus the results from Mombarg et al. (2019). The grey dashed lines indicate perfect concordance.

\begin{tabular}{c|cccc}
\hline \hline & $\begin{array}{c}\text { Asteroseismic } \\
\text { input }\end{array}$ & $\begin{array}{c}\text { Spectroscopic } \\
T_{\text {eff }} \text { and } \log g\end{array}$ & $Z$ & Gaia $L$ \\
\hline This work & $P_{n}$ & $\begin{array}{c}\text { A posteriori } \\
\text { cutoff }\end{array}$ & $\begin{array}{c}\text { Fixed to value } \\
\text { from spectroscopy }\end{array}$ & $\begin{array}{c}\text { A posteriori } \\
\text { cutoff }\end{array}$
\end{tabular}

Mombarg et al. (2019)

$\Pi_{0}$

Fitted

Fixed to 0.014

Not included

Table 4. Comparison between the method used in Mombarg et al. (2019) and this work.

Rossby modes, but many of these stars lack spectroscopic observations therefore the MLEs may in general be less constrained.

To compute the mode periods, we have relied on the Traditional Approximation of Rotation (TAR), which assumes spherical symmetry and loses its validity for rapidly rotating stars deformed by the centrifugal force (cf. Mathis \& Prat 2019, for an improved description of the TAR in that case). In Figure 21, the distribution of the critical Roche frequency for the stars in our sample is shown. Inspecting the quality of fit for the star with the largest fraction of $\Omega / \Omega_{\text {crit,Roche }}$, namely KIC 12066947 ( $\sim 80 \%)$, suggests the TAR is still able to reproduce the observed patterns to a satisfactorily level, in line with the findings by Henneco et al. (2021). However, our models are not able the reproduce the sharp dip in the observed pattern, suggesting its caused by resonances between gravito-inertial and pure-inertial modes (Ouazzani et al. 2020; Saio et al. 2021). An improved formalism of the TAR, taking into account the centrifugal deformation, has been developed by Mathis \& Prat (2019). The inclusion of the centrifugal force in the pulsation computations of $\gamma$ Dor stars has a smaller effect on the predicted values for the periods than the inclusion of stratified envelope mixing profiles due to radiative levitation (Mombarg et al. 2020) or other mixing phenomena, although for lower radial orders the effect of the centrifugal force should be observable in long time-base, space-based photometry Henneco et al. (2021).

In the stellar structure and evolution models used in this work, the effect of atomic diffusion is neglected. Mombarg et al. (2020) have demonstrated for two slowly-rotating $\gamma$ Dor stars that this process (including radiative levitations) can lead to significant differences in the derived mass, age, and near-core boundary mixing. The obtained period spacing pattern of the best-fitting model for KIC 9751996 suggests that we are underestimating $D_{0}$. The need for more envelope mixing supports the conclusion of Mombarg et al. (2020) who found that models without atomic diffusion better reproduce the observed mode periods for this particular star, if other forms of envelope mixing are able to counteract the effects of atomic diffusion. Even though the use of a neural network greatly decreases the number of required stellar models in the modelling, doing the full radia-

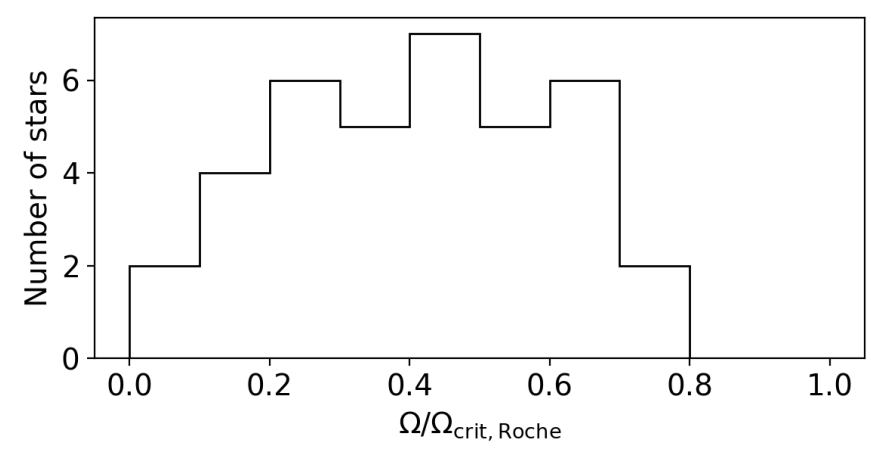

Fig. 21. Distribution of the rotation frequency as a fraction of the critical rotation frequency in the Roche framework. Rotation frequencies are taken from Van Reeth et al. $(2016,2018)$.

tive levitation calculations in MESA still requires a large amount of computation time. The modelling of the sample in this work with atomic diffusion (including radiative levitation), as well as a non-constant parametrised mixing profile in the radiative envelope will be taken up in a future paper.

Acknowledgements. The research leading to these results has received funding from the European Research Council (ERC) under the European Unions Horizon 2020 research and innovation programme (grant agreement $\mathrm{N}^{\circ} 670519$ : MAMSIE) and from the KU Leuven Research Council (grant C16/18/005: PARADISE). The computational resources and services used in this work were provided by the VSC (Flemish Supercomputer Center), funded by the Research Foundation - Flanders (FWO) and the Flemish Government department EWI. TVR gratefully acknowledges support from the Research Foundation Flanders (FWO) through grant 12ZB620N. We thank Dr. Dominic M. Bowman for his comments on the manuscript.

\section{References}

Aerts, C. 2021, Reviews of Modern Physics, 93, 015001

Aerts, C., Mathis, S., \& Rogers, T. M. 2019, ARA\&A, 57, 35

Aerts, C., Molenberghs, G., Michielsen, M., et al. 2018, ApJS, 237, 15

Aerts, C., Van Reeth, T., \& Tkachenko, A. 2017, ApJ, 847, L7

Antoci, V., Cunha, M. S., Bowman, D. M., et al. 2019, MNRAS, 490, 4040

Asplund, M., Grevesse, N., Sauval, A. J., \& Scott, P. 2009, ARA\&A, 47, 481 
Bailer-Jones, C. A. L., Rybizki, J., Fouesneau, M., Mantelet, G., \& Andrae, R. 2018, AJ, 156, 58

Beck, P. G., Montalban, J., Kallinger, T., et al. 2012, Nature, 481, 55

Bellinger, E. P., Angelou, G. C., Hekker, S., et al. 2016, ApJ, 830, 31

Bishop, C. 1995, Neural networks for pattern recognition (Oxford University Press, USA)

Borucki, W. J., Koch, D., Basri, G., et al. 2010, Science, 327, 977

Buysschaert, B., Aerts, C., Bowman, D. M., et al. 2018, A\&A, 616, A148

Cantiello, M., Mankovich, C., Bildsten, L., Christensen-Dalsgaard, J., \& Paxton, B. 2014, ApJ, 788, 93

Christophe, S., Ballot, J., Ouazzani, R. M., Antoci, V., \& Salmon, S. J. A. J. 2018, A\&A, 618, A47

Claret, A. \& Torres, G. 2017, ApJ, 849, 18

Deal, M., Goupil, M. J., Marques, J. P., Reese, D. R., \& Lebreton, Y. 2020, A\&A, 633, A23

Deheuvels, S., Brandão, I., Silva Aguirre, V., et al. 2016, A\&A, 589, A93

den Hartogh, J. W., Eggenberger, P., \& Deheuvels, S. 2020, A\&A, 634, L16

den Hartogh, J. W., Eggenberger, P., \& Deheuvels, S. 2020, A\&A, 634, 116

den Hartogh, J. W., Eggenberger, P., \& Hirschi, R. 2019, A\&A, 622, A187

Dupret, M. A., Grigahcène, A., Garrido, R., Gabriel, M., \& Scuflaire, R. 2004, A\&A, 414, L17

Eckart, C. 1960, Physics of Fluids, 3, 421

Eggenberger, P., Deheuvels, S., Miglio, A., et al. 2019a, A\&A, 621, A66

Eggenberger, P., den Hartogh, J. W., Buldgen, G., et al. 2019b, A\&A, 631, L6

Eggenberger, P., Lagarde, N., Miglio, A., et al. 2017, A\&A, 599, A18

Freytag, B., Ludwig, H. G., \& Steffen, M. 1996, A\&A, 313, 497

Fuller, J., Lecoanet, D., Cantiello, M., \& Brown, B. 2014, ApJ, 796, 17

Fuller, J., Piro, A. L., \& Jermyn, A. S. 2019, MNRAS, 485, 3661

Gehan, C., Mosser, B., Michel, E., Samadi, R., \& Kallinger, T. 2018, A\&A, 616, A24

Glorot, X., Bordes, A., \& Bengio, Y. 2011, in (Fort Lauderdale, FL, USA: JMLR Workshop and Conference Proceedings), 315-323

Green, G. M., Schlafly, E. F., Finkbeiner, D., et al. 2018, MNRAS, 478, 651

Guzik, J. A., Kaye, A. B., Bradley, P. A., Cox, A. N., \& Neuforge, C. 2000, ApJ, 542, L57

Hendriks, L. \& Aerts, C. 2019, Publications of the Astronomical Society of the Pacific, 131, 108001

Henneco, J., Van Reeth, T., Prat, V., et al. 2021, arXiv e-prints, arXiv:2101.04116

Hermes, J. J., Gänsicke, B. T., Kawaler, S. D., et al. 2017, ApJS, 232, 23

Hon, M., Bellinger, E. P., Hekker, S., Stello, D., \& Kuszlewicz, J. S. 2020, arXiv e-prints, arXiv:2009.06972

Keen, M. A., Bedding, T. R., Murphy, S. J., et al. 2015, MNRAS, 454, 1792

Kurtz, D. W., Saio, H., Takata, M., et al. 2014, MNRAS, 444, 102

Lee, U. \& Baraffe, I. 1995, A\&A, 301, 419

Li, G., Bedding, T. R., Murphy, S. J., et al. 2019a, MNRAS, 482, 1757

Li, G., Van Reeth, T., Bedding, T. R., et al. 2019b, MNRAS, 2517

Li, G., Van Reeth, T., Bedding, T. R., et al. 2020, MNRAS, 491, 3586

Maeder, A. 2009, Physics, Formation and Evolution of Rotating Stars

Mathis, S. \& Prat, V. 2019, A\&A, 631, A26

Michielsen, M., Pedersen, M. G., Augustson, K. C., Mathis, S., \& Aerts, C. 2019, A\&A, 628, A76

Miglio, A., Montalbán, J., Noels, A., \& Eggenberger, P. 2008, MNRAS, 386, 1487

Mombarg, J. S. G., Dotter, A., Van Reeth, T., et al. 2020, ApJ, 895, 51

Mombarg, J. S. G., Van Reeth, T., Pedersen, M. G., et al. 2019, MNRAS, 485, 3248

Moravveji, E., Aerts, C., Pápics, P. I., Triana, S. A., \& Vandoren, B. 2015, A\&A, 580, A27

Moravveji, E., Townsend, R. H. D., Aerts, C., \& Mathis, S. 2016, ApJ, 823, 130

Mosser, B., Goupil, M. J., Belkacem, K., et al. 2012, A\&A, 548, A10

Ouazzani, R.-M., Lignières, F., Dupret, M.-A., et al. 2020, arXiv e-prints, arXiv:2006.09404

Ouazzani, R. M., Marques, J. P., Goupil, M. J., et al. 2019, A\&A, 626, A121

Ouazzani, R.-M., Salmon, S. J. A. J., Antoci, V., et al. 2017, MNRAS, 465, 2294

Paxton, B., Bildsten, L., Dotter, A., et al. 2011, ApJS, 192, 3

Paxton, B., Cantiello, M., Arras, P., et al. 2013, ApJS, 208, 4

Paxton, B., Marchant, P., Schwab, J., et al. 2015, ApJS, 220, 15

Paxton, B., Schwab, J., Bauer, E. B., et al. 2018, ApJS, 234, 34

Paxton, B., Smolec, R., Schwab, J., et al. 2019, ApJS, 243, 10

Pedersen, M. G., Aerts, C., Pápics, P. I., et al. 2021, Nature Astronomy, in press

Pedersen, M. G., Aerts, C., Pápics, P. I., \& Rogers, T. M. 2018, A\&A, 614, A128

Prat, V., Mathis, S., Buysschaert, B., et al. 2019, A\&A, 627, A64

Ricker, G. R., Winn, J. N., Vanderspek, R., et al. 2015, Journal of Astronomical Telescopes, Instruments, and Systems, 1, 014003

Rogers, T. M. \& McElwaine, J. N. 2017, ApJ, 848, L1

Saio, H., Bedding, T. R., Kurtz, D. W., et al. 2018, MNRAS, 477, 2183

Saio, H., Kurtz, D. W., Takata, M., et al. 2015, MNRAS, 447, 3264

Saio, H., Takata, M., Lee, U., Li, G., \& Van Reeth, T. 2021, MNRAS, 502, 5856

Salaris, M. \& Cassisi, S. 2017, Royal Society Open Science, 4, 170192

Tayar, J. \& Pinsonneault, M. H. 2018, ApJ, 868, 150

Tayar, J. \& Pinsonneault, M. H.
Torres, G. 2010, AJ, 140,1158

Torres, G. 2010, AJ, 140, 1158
Townsend, R. H. D. 2003, MNRAS, 340, 1020

Townsend, R. H. D., Goldstein, J., \& Zweibel, E. G. 2018, MNRAS, 475, 879

Townsend, R. H. D. \& Teitler, S. A. 2013, MNRAS, 435, 3406

Van Beeck, J., Prat, V., Van Reeth, T., et al. 2020, A\&A, 638, A149

Van Reeth, T., Mombarg, J. S. G., Mathis, S., et al. 2018, A\&A, 618, A24

Van Reeth, T., Tkachenko, A., \& Aerts, C. 2016, A\&A, 593, A120

Van Reeth, T., Tkachenko, A., Aerts, C., et al. 2015a, ApJS, 218, 27

Van Reeth, T., Tkachenko, A., Aerts, C., et al. 2015a, ApJS, 218, 27

Van Reeth, T., Tkachenko, A., Aerts, C., et al. 2015b, ApJS, 218, 27
Verma, K., Raodeo, K., Basu, S., et al. 2019, MNRAS, 483, 4678

Xiong, D. R., Deng, L., Zhang, C., \& Wang, K. 2016, MNRAS, 457, 3163 
Appendix A: luminosities from Gaia

\begin{tabular}{|c|c|c|c|c|c|c|}
\hline KIC & $m_{\mathrm{V}}$ & $\mathrm{BC}_{\mathrm{V}}$ & $\overline{D(\mathrm{pc})}$ & $A_{\mathrm{V}}$ & Reliable $A_{\mathrm{V}}$ ? & $\log L / \mathrm{L}_{\odot}$ \\
\hline 2710594 & 11.79 & 0.031 & $643_{-9}^{+9}$ & 0.21 & $\mathrm{Y}$ & $0.864_{-0.075}^{+0.075}$ \\
\hline 3448365 & 9.92 & 0.031 & $261_{-2}^{+2}$ & 0.05 & $\mathrm{~N}$ & $0.766_{-0.035}^{+0.035}$ \\
\hline 4846809 & 12.36 & 0.035 & $852_{-22}^{+23}$ & 0.16 & $\mathrm{Y}$ & $0.86_{-0.023}^{+0.023}$ \\
\hline 5114382 & 11.55 & 0.034 & $618_{-10}^{+11}$ & 0.27 & $\mathrm{Y}$ & $0.948_{-0.046}^{+0.033}$ \\
\hline $5522154 *$ & 10.44 & 0.028 & $285_{-2}^{+2}$ & 0.0 & $\mathrm{~N}$ & $0.615_{-0.072}^{+0.040}$ \\
\hline $5708550 *$ & 11.93 & 0.03 & $641_{-13}^{+23}$ & 0.15 & $\mathrm{Y}$ & $0.782_{-0.071}^{+0.012}$ \\
\hline 5788623 & 12.07 & 0.034 & $649_{-12}^{+13}$ & 0.12 & $\mathrm{Y}$ & $0.722^{+0.0025}$ \\
\hline 6468146 & 9.96 & 0.035 & $326_{-3}^{+12}$ & 0.02 & $\mathrm{~N}$ & $0.927_{-0.015}^{-0.024}$ \\
\hline 6468987 & 12.67 & 0.034 & $910^{+3}$ & 0.54 & Y & $0.947^{+0.0833}$ \\
\hline $6678174 *$ & 11.74 & 0.033 & $686_{-12}^{+13}$ & 0.21 & $\mathrm{~N}$ & $0.938_{-0.048}^{+0.0048}$ \\
\hline 6935014 & 10.91 & 0.033 & $395_{-3}^{+12}$ & 0.15 & $\mathrm{Y}$ & $0.767_{-0.03}^{-0.048}$ \\
\hline $6953103^{*}$ & 12.56 & 0.035 & $781_{-15}^{+16}$ & 0.27 & $\mathrm{Y}$ & $0.748_{-0.016}^{+0.016}$ \\
\hline 7023122 & 10.84 & 0.035 & $389_{-4}^{-15}$ & 0.05 & $\mathrm{~N}$ & $0.744_{-0.006}^{+0.016}$ \\
\hline $7365537^{*}$ & 9.19 & 0.035 & $178_{-1}^{+1}$ & 0.0 & $\mathrm{~N}$ & $0.704_{-0.004}^{+0.006}$ \\
\hline 7380501 & 11.98 & 0.029 & $663_{-10}^{+11}$ & 0.46 & $\mathrm{Y}$ & $0.917_{-0.114}^{+0.0114}$ \\
\hline 7434470 & 12.02 & 0.031 & $602_{-9}^{+9}$ & 0.25 & $\mathrm{Y}$ & $0.73_{-0.065}^{+0.065}$ \\
\hline 7583663 & 12.69 & 0.031 & $972_{-25}^{+96}$ & 0.21 & $\mathrm{Y}$ & $0.862_{-0.151}^{+0.151}$ \\
\hline 7939065 & 12.14 & 0.035 & $696_{-15}^{+15}$ & 0.2 & $\mathrm{Y}$ & $0.787_{-0.021}^{+0.021}$ \\
\hline 8364249 & 11.95 & 0.032 & $806_{-15}^{+15}$ & 0.25 & $\mathrm{Y}$ & $1.011_{-0.085}^{+0.021}$ \\
\hline 8375138 & 11.02 & 0.032 & $422_{-4}^{-13}$ & 0.13 & $\mathrm{~N}$ & $0.775_{-0.04}^{+0.04}$ \\
\hline 8645874 & 9.92 & 0.035 & $274_{-2}^{+2}$ & 0.02 & $\mathrm{~N}$ & $0.795_{-0.015}^{+0.045}$ \\
\hline 8836473 & 12.78 & 0.035 & $926_{-21}^{+22}$ & 0.56 & $\mathrm{Y}$ & $0.923_{-0.033}^{+0.0033}$ \\
\hline 9480469 & 12.78 & 0.035 & $908_{-18}^{+18}$ & 0.31 & $\mathrm{Y}$ & $0.806_{-0.037}^{+0.037}$ \\
\hline 9595743 & 12.09 & 0.035 & $618_{-7}^{+8}$ & 0.06 & $\mathrm{Y}$ & $0.649_{-0.019}^{+0.0019}$ \\
\hline 9751996 & 10.96 & 0.034 & $\begin{array}{l}588_{-9}^{+9} \\
\end{array}$ & 0.1 & $\mathrm{Y}$ & $1.073_{-0.049}^{+0.019}$ \\
\hline 10467146 & 12.66 & 0.034 & $1114_{-41}^{+45}$ & 0.29 & $\mathrm{Y}$ & $1.027_{-0.082}^{+0.049}$ \\
\hline 11080103 & 12.94 & 0.035 & $1017_{-25}^{+24}$ & 0.2 & $\mathrm{Y}$ & $0.797_{-0.017}^{+0.08 \%}$ \\
\hline 11099031 & 10.02 & 0.025 & $298_{-2}^{+\overline{2}^{-2}}$ & 0.02 & $\mathrm{~N}$ & $0.831_{-0.093}^{+0.093}$ \\
\hline 11294808 & 11.73 & 0.03 & $679_{-8}^{+8}$ & 0.21 & $\mathrm{Y}$ & $0.939_{-0.085}^{+0.0085}$ \\
\hline 11456474 & 12.49 & 0.032 & $864_{-17}^{+18}$ & 0.2 & $\mathrm{Y}$ & $0.836_{-0.098}^{+0.0088}$ \\
\hline 11721304 & 11.71 & 0.034 & $550_{-7}^{+1}$ & 0.13 & $\mathrm{Y}$ & $0.726_{-0.024}^{+0.008}$ \\
\hline 11754232 & 12.24 & 0.035 & $799_{-21}^{+22}$ & 0.08 & $\mathrm{Y}$ & $0.821_{-0.019}^{+0.024}$ \\
\hline 11826272 & 10.21 & 0.031 & $341_{-5}^{+5}$ & 0.04 & $\mathrm{~N}$ & $0.877_{-0.048}^{+0.019}$ \\
\hline 11907454 & 11.38 & 0.032 & $469_{-5}^{+5}$ & 0.05 & $\mathrm{Y}$ & $0.688_{-0.045}^{+0.049}$ \\
\hline 11917550 & 11.12 & 0.033 & $448_{-5}^{+5}$ & 0.05 & $\mathrm{~N}$ & $0.754_{-0.035}^{+0.0435}$ \\
\hline 11920505 & 9.88 & 0.034 & $243_{-1}^{+3}$ & 0.0 & $\mathrm{~N}$ & $0.698_{-0.013}^{+0.813}$ \\
\hline 12066947 & 10.23 & 0.035 & $325_{-3}^{+3}$ & 0.0 & $\mathrm{~N}$ & $0.812_{-0.006}^{+0.006}$ \\
\hline
\end{tabular}

Table A.1. Visual apparent magnitudes $m_{\mathrm{V}}$, bolometric corrections $\mathrm{BC}_{\mathrm{V}}$, distances $D$ (Bailer-Jones et al. 2018), and extinctions $A_{\mathrm{V}}$ used to derive the luminosity. The 'Reliable $A_{\mathrm{V}}$ ' flag indicates whether a reliable estimation of the extinction could be made with the Bayerstar2019 extinction map. Stars for which no reliable estimation could be made according to the Bayerstar2019 map are relatively close by and therefore the extinction is expected to be minimal. For the stars marked with an asterisk (*) the luminosity is inconsistent with the pulsations and is therefore not used in the modelling. 
Appendix B: Best-fitting parameters from neural network

\begin{tabular}{|c|c|c|c|}
\hline$\overline{\mathrm{KIC}}$ & $\overline{M_{\star}\left[\mathrm{M}_{\odot}\right]}$ & $\overline{X_{\mathrm{c}}}$ & $\overline{\overline{f_{\mathrm{ov}}}}$ \\
\hline 2710594 & $1.495_{-0.093}^{+0.128}$ & $0.482_{-0241}^{+0.108}$ & $0.0265_{-0.0364}^{+0.0035}$ \\
\hline 3448365 & $1.485_{-0.075}^{+0.010}$ & $0.459_{-01010}^{+0.171}$ & $0.0271_{-0.0029}^{+0.0029}$ \\
\hline 4846809 & $1.487_{-0.049}^{+0.049}$ & $0.409_{-0.070}^{+0.062}$ & $0.0206^{+0.00037}$ \\
\hline 5114382 & $1.613_{-0}^{+0}$ & $0.390_{-0}^{+0}$ & $0.0222_{-c}^{+c}$ \\
\hline 5522154 & $1.723_{-0 .}^{+0.2}$ & $0.554_{-0}^{+0}$ & $0.0253^{+0}$ \\
\hline 5708550 & $1.990_{-0610}^{+0.010}$ & $0.137_{-0}^{+0}$ & $0.0165^{+0.00135}$ \\
\hline 5788623 & $1.474_{-0.052}^{+0.001}$ & $0.505_{-0}^{+0}$ & $0.0102_{-0.000}^{+0.0056}$ \\
\hline 6468146 & $1.594^{+0.010}$ & $0.444^{+0.0014}$ & $0.0205^{+0.00}$ \\
\hline 6468987 & $1.568_{-0.159}^{+0.134}$ & $0.522^{+0 .}$ & $0.0274_{-0 .}^{+0 .}$ \\
\hline 6678174 & $1.768_{-02}^{+0 .}$ & $0.650^{+0}$ & $0.0157^{+0}$ \\
\hline 6935014 & $1.452^{+0.029}$ & $0.613^{+0}$ & $0.0293^{+0}$ \\
\hline 6953103 & $1.877_{-0194}^{+0.121}$ & $0.339^{+}$ & $0.0280_{-0}^{+0}$ \\
\hline 7023122 & $1.515^{+0.004}$ & $0.697^{-}$ & $0.0101^{+t}$ \\
\hline 7365537 & $1.700^{+0.3000}$ & $0.578^{+0}$ & $0.0188^{+0.01112}$ \\
\hline 7380501 & $1.717_{-0.034}^{+0.060}$ & $0.138_{-0}^{+0 .}$ & $0.0107^{-0.0}$ \\
\hline 7434470 & $1.483^{-0.014}$ & $0.449^{-0}$ & $0.0263^{+0}$ \\
\hline 7583663 & $1.467_{-01}^{+0.2}$ & $0.450_{-0}^{+0}$ & $0.0204^{+0}$ \\
\hline 7939065 & $1.479_{-0}^{+0}$ & $0.465_{-0}^{+0}$ & $0.0193_{-0}^{+0}$ \\
\hline 8364249 & $1.519^{-0.062}$ & $0.452^{-0}$ & $0.0275^{+0}$ \\
\hline 8375138 & $1.469^{+0.048}$ & $0.499^{+0.12124}$ & $0.0116_{-0.0184}^{+0.0184}$ \\
\hline 8645874 & $1.518^{+0.0}$ & $0.639^{+0}$ & $0.0297^{+0}$ \\
\hline 8836473 & $1.482^{+0.1}$ & $0.315^{+0}$ & $0.0111_{-0.0142}^{+0.0142}$ \\
\hline 9480469 & $1.487^{-}$ & $0.383_{-0.072}^{+0.196}$ & $0.0231_{-}^{+}$ \\
\hline 9595743 & $1.424^{+0}$ & $0.630^{+0.0042}$ & $0.0107^{+}$ \\
\hline 9751996 & $1.740^{+0.0009}$ & $0.241^{+0}$ & $0.0124^{+0.0121}$ \\
\hline 10467146 & $1.768_{-0.27}^{+0.021}$ & $0.161_{-0.02}^{+0.256}$ & $0.0115_{-0 .}^{+0 .}$ \\
\hline 11080103 & $1.528^{+0.014}$ & $0.545^{+0}$ & $0.0175^{+c}$ \\
\hline 11099031 & $1.572_{-0215}^{+0.038}$ & $0.290_{-0.071}^{+0.284}$ & $0.0299^{+\zeta}$ \\
\hline 11294808 & $1.450_{-0.045}^{+0.205}$ & $0.291_{-0101}^{+0.194}$ & $0.0101_{-c}^{+c}$ \\
\hline 11456474 & $1.487^{+0.141}$ & $0.387^{+0.121}$ & $0.0127^{+0.01000134}$ \\
\hline 11721304 & $1.431_{-0.038}^{+0.038}$ & $0.633^{+}$ & $0.0104_{-00004}^{+0.0196}$ \\
\hline 11754232 & $1.532^{+0.0}$ & $0.641^{+0}$ & $0.0102^{+0.00196}$ \\
\hline 11826272 & $1.524^{+0.0}$ & $0.364^{+0}$ & $0.0172^{+0.00019}$ \\
\hline 11907454 & $1.465_{-0102}^{+0.012}$ & $0.597_{-01702}^{+0.102}$ & $0.0104_{-0}^{+0}$ \\
\hline 11917550 & $1.512_{-0.099}^{+0.001}$ & $0.486_{-0.076}^{+0.176}$ & $0.0192_{-}^{+}$ \\
\hline 11920505 & $1.445_{-0.013}^{+0.026}$ & $0.602_{-0.082}^{+0.035}$ & $0.0101_{-0.001}^{+0.01126}$ \\
\hline 12066947 & $1.490_{-}^{+}$ & $0.475_{-}^{+}$ & $0.0186_{-t}^{+}$ \\
\hline
\end{tabular}

Table B.1. Best-fitting parameters of the model predicted by the C-3P0 neural network.

\section{Appendix C: Distribution of the residuals}

In Figure C.1 we show the residuals $\left(P_{\text {true }}-P_{\text {pred }}\right)-$ per radial order - of the NN on the grid of 38915 stellar pulsation models used for training and validation.

\section{Appendix D: Best-matching period spacing patterns}

In this appendix we present the best-fitting period spacing patterns found with C-3PO (in red) and GYRE (in blue) for all stars in our sample. The observed period spacing patterns are taken from Van Reeth et al. (2015b), where the uncertainties are typically smaller than the symbol size. Missing radial orders are

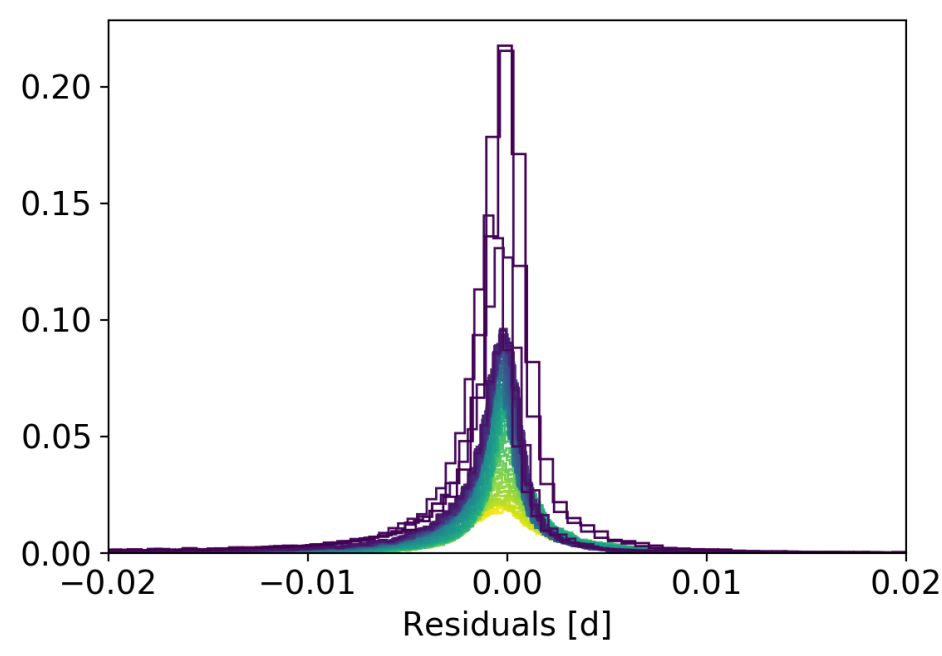

Fig. C.1. Normalized distributions of the residuals of the $\mathrm{NN}$ on the grid of pulsation models used for training and validation. Darker colors indicate higher radial orders.

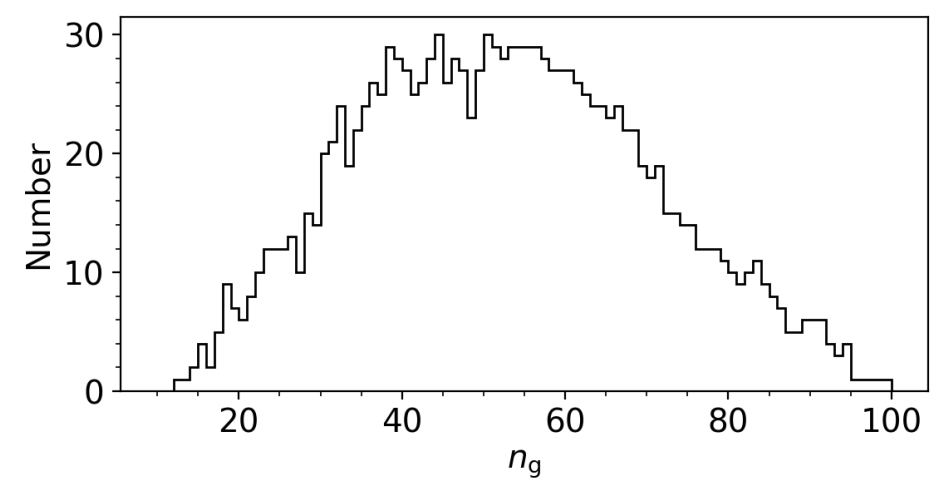

Fig. D.1. The distribution of the identified radial orders for all stars in our sample.

indicated by dashed lines. As only the mode periods are fitted, these values are also indicated by dashes at the bottom/top of each panel. Furthermore, in Fig D.1 we show the distribution of radial orders. A similar distribution is found compared to the one from Li et al. (2020) (their Figure 17). 


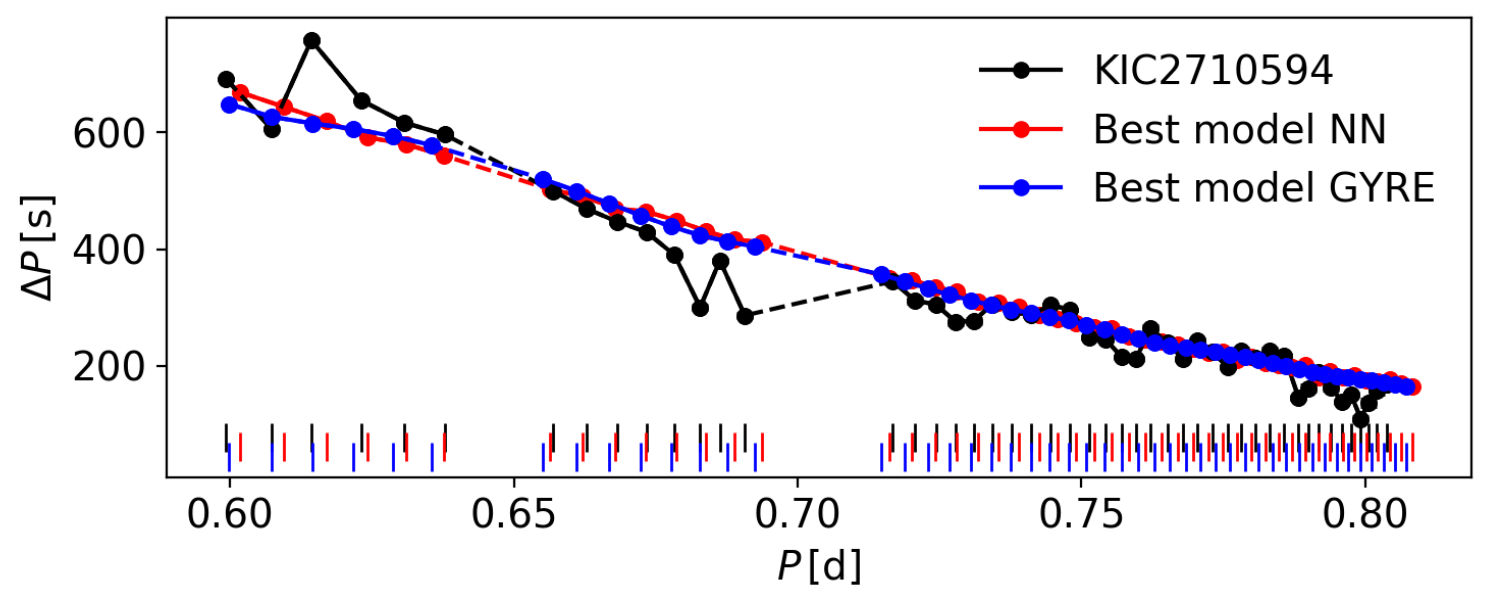

Fig. D.2. Best-fitting models from the neural network and GYRE for KIC 2710594.

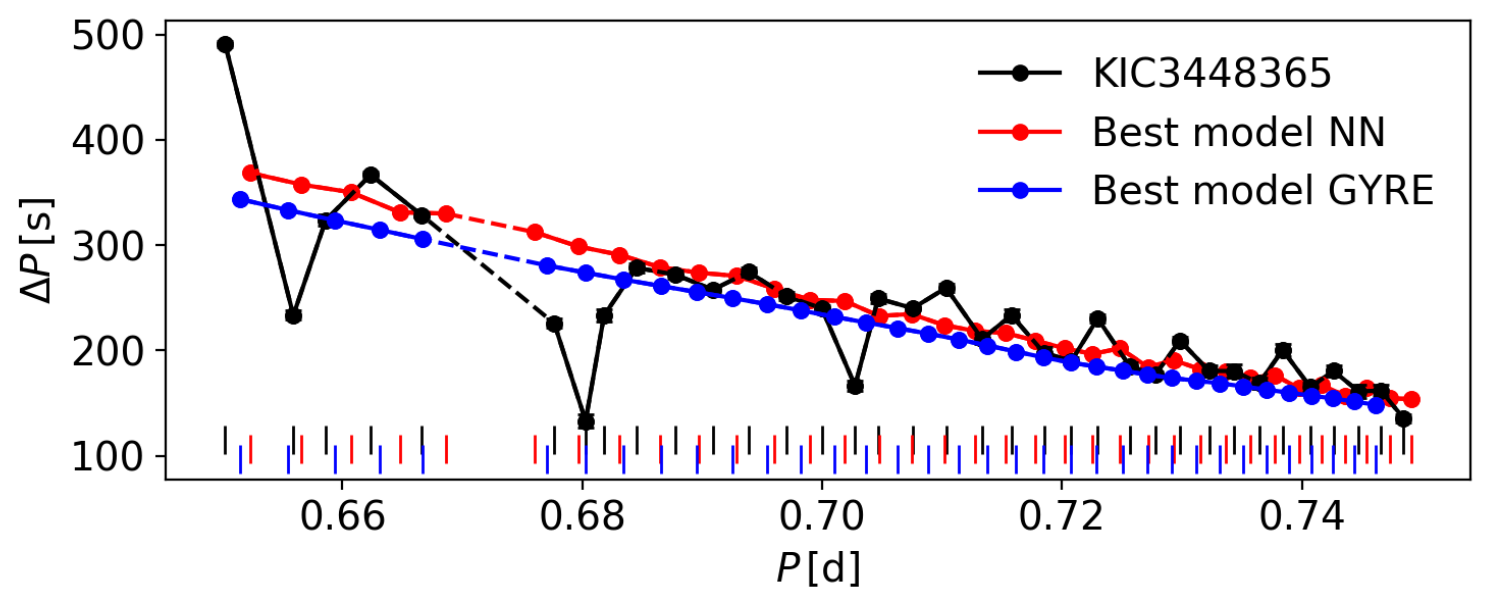

Fig. D.3. Best-fitting models from the neural network and GYRE for KIC 3448365 . 

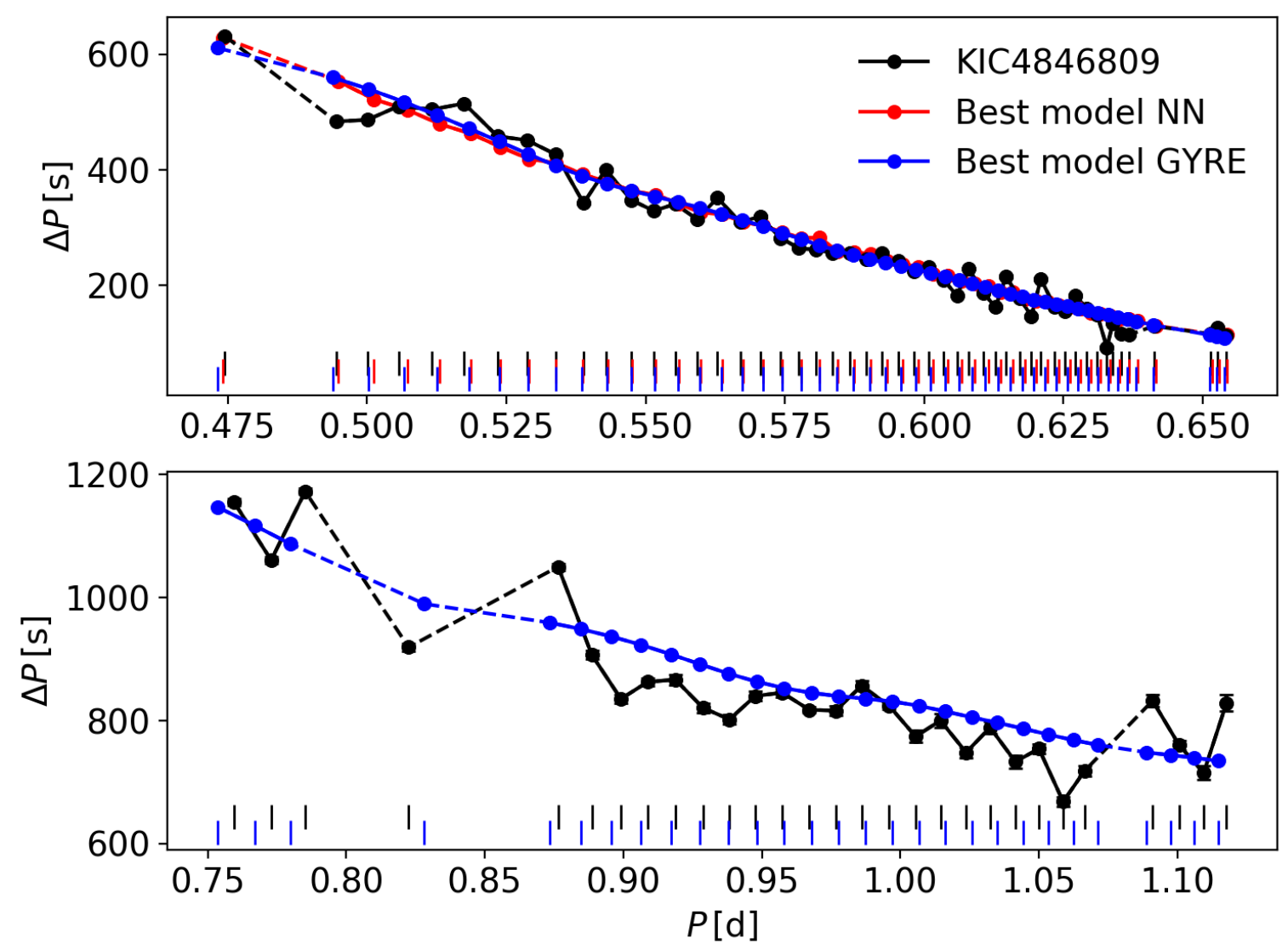

Fig. D.4. Best-fitting models from the neural network and GYRE for KIC 4846809. Top: $(\ell, m)=(1,1)$, bottom $(\ell, m)=(1,0)$.

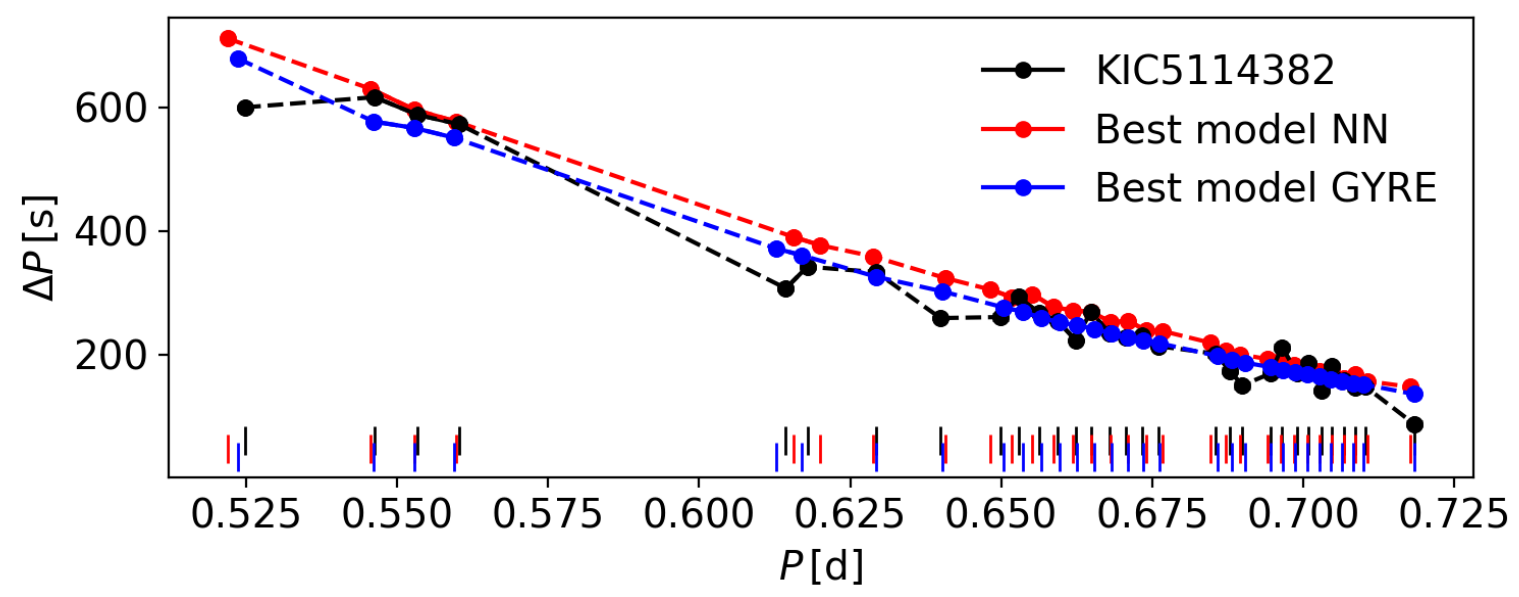

Fig. D.5. Best-fitting models from the neural network and GYRE for KIC 5114382. 


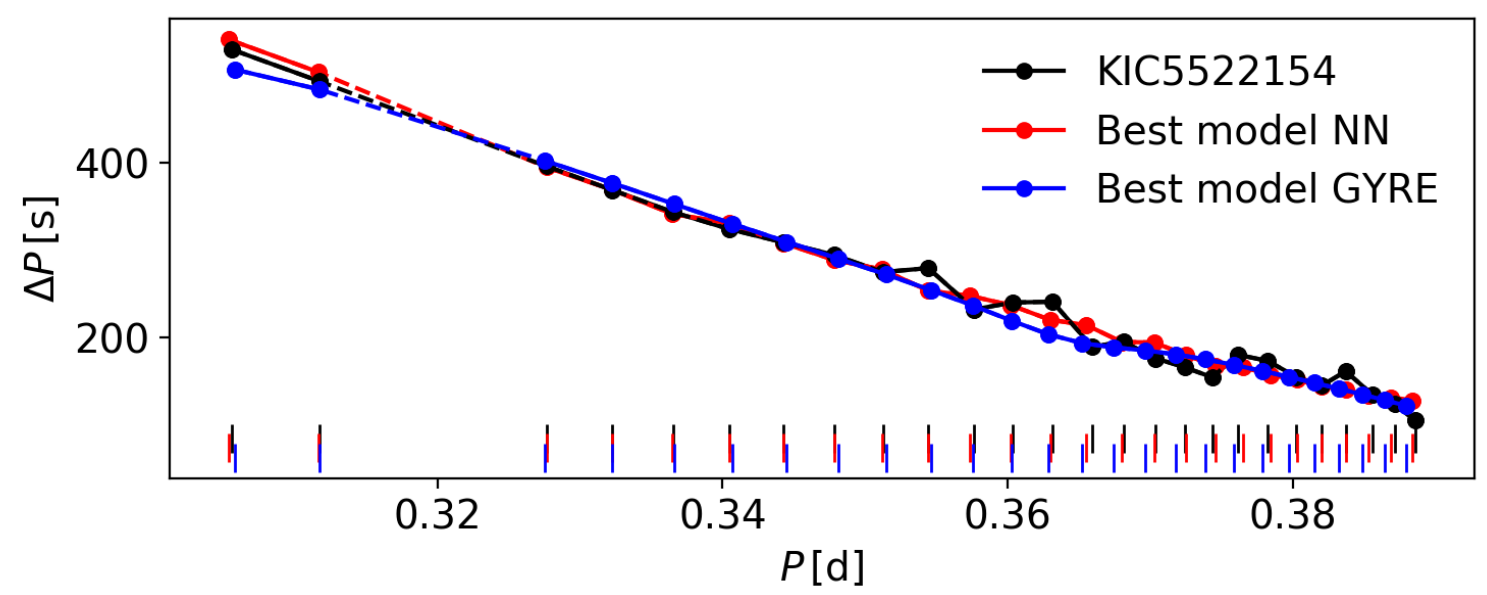

Fig. D.6. Best-fitting models from the neural network and GYRE for KIC 5522154 .

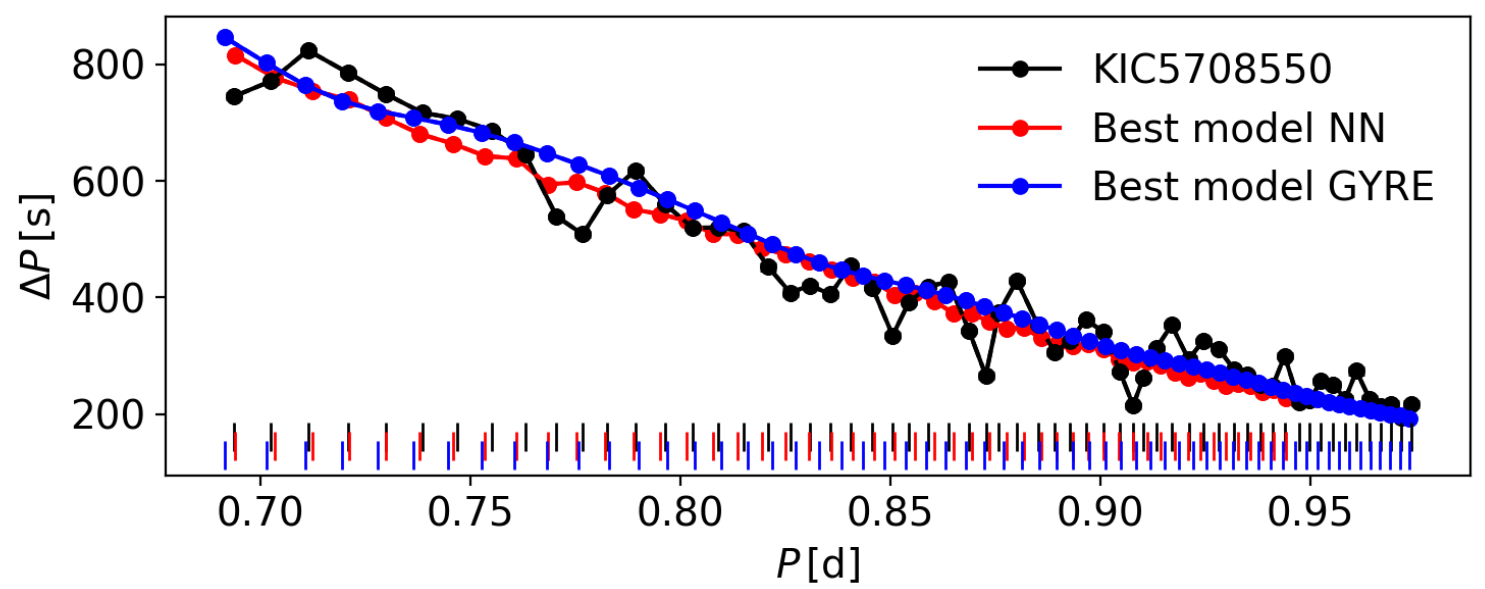

Fig. D.7. Best-fitting models from the neural network and GYRE for KIC 5708550.

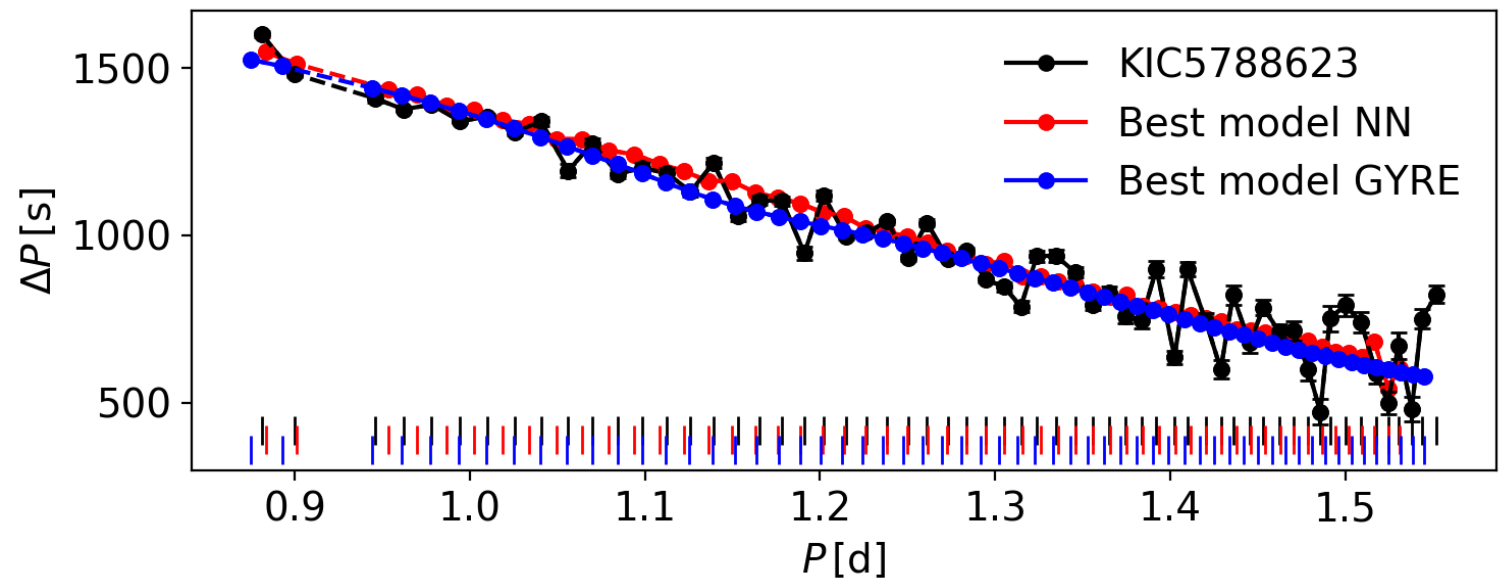

Fig. D.8. Best-fitting models from the neural network and GYRE for KIC 5788623. 


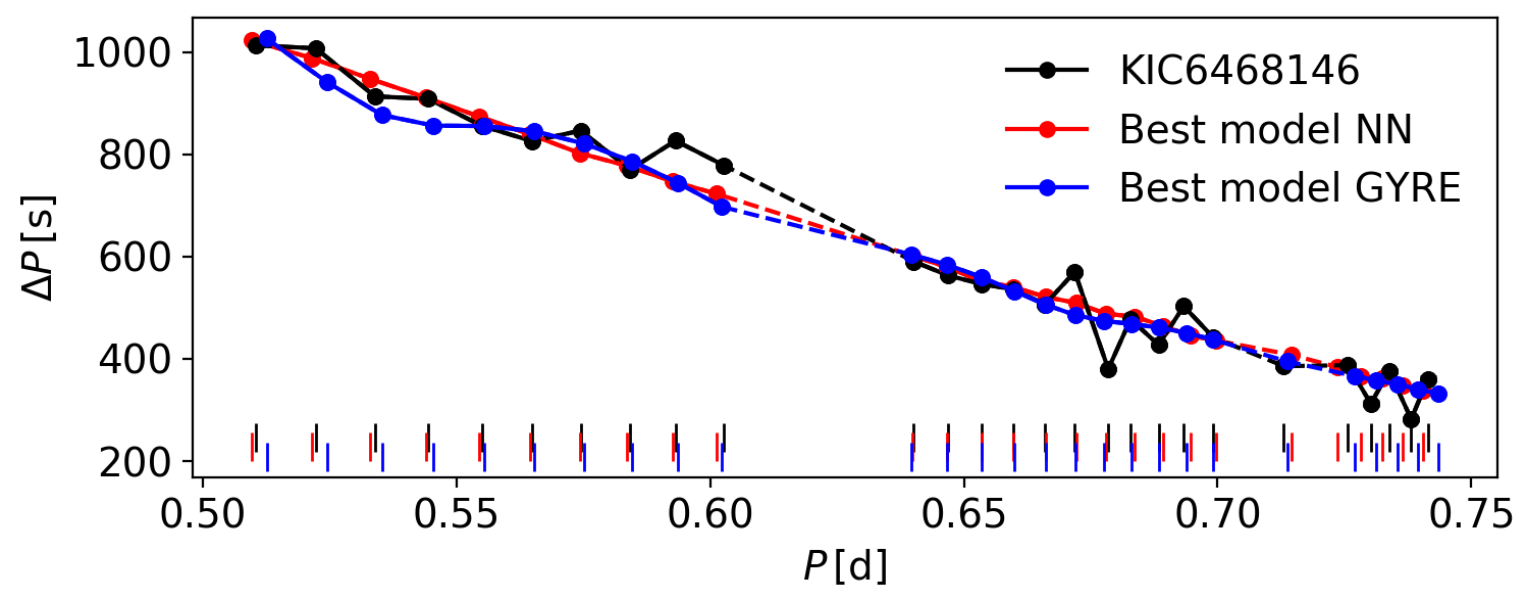

Fig. D.9. Best-fitting models from the neural network and GYRE for KIC 6468146.

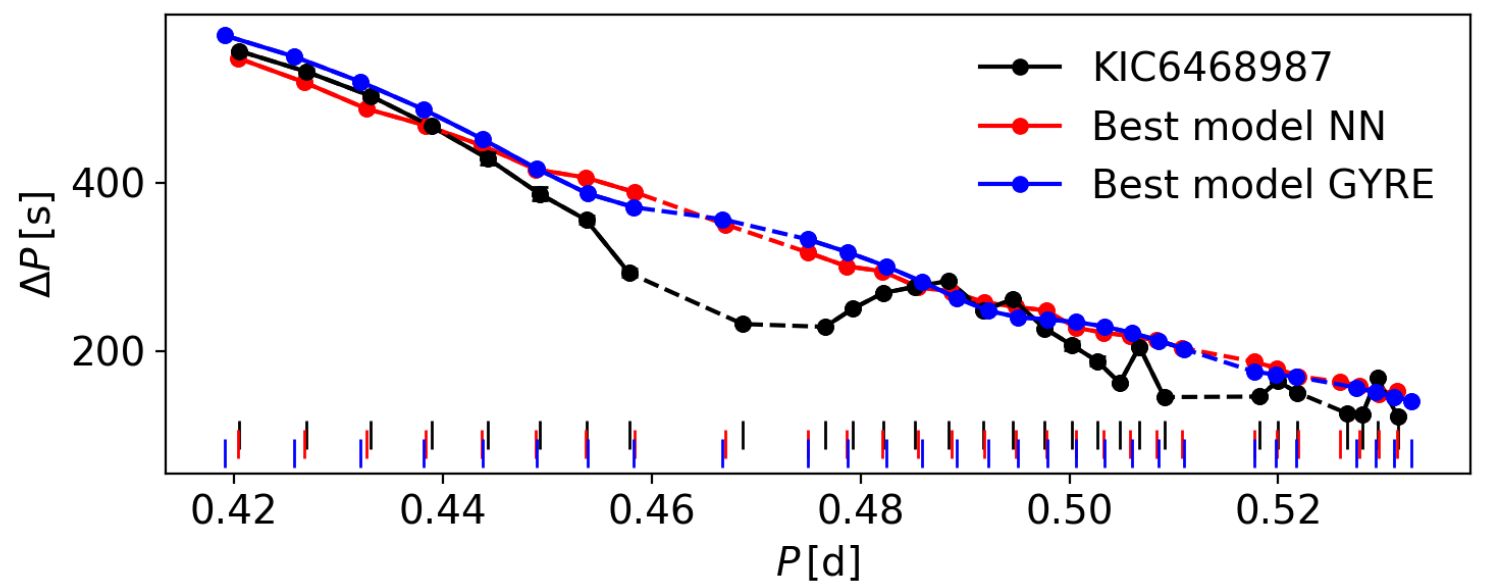

Fig. D.10. Best-fitting models from the neural network and GYRE for KIC 6468987.

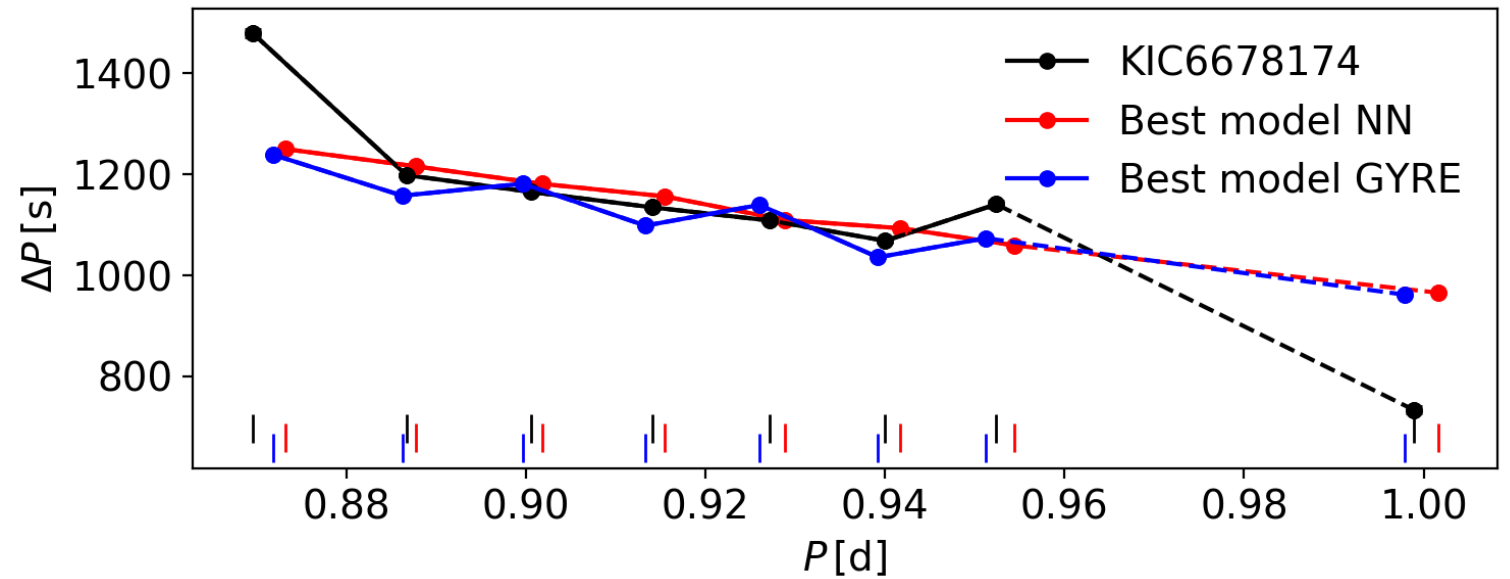

Fig. D.11. Best-fitting models from the neural network and GYRE for KIC 6678174. 


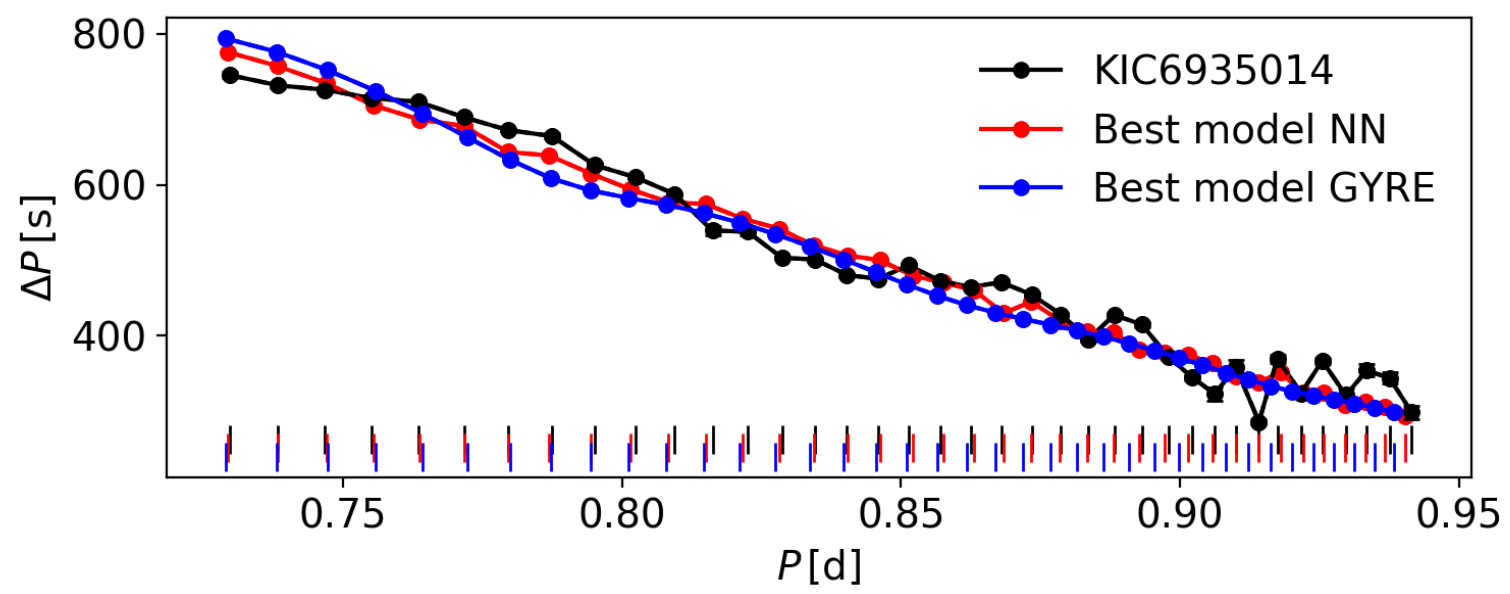

Fig. D.12. Best-fitting models from the neural network and GYRE for KIC 6935014.

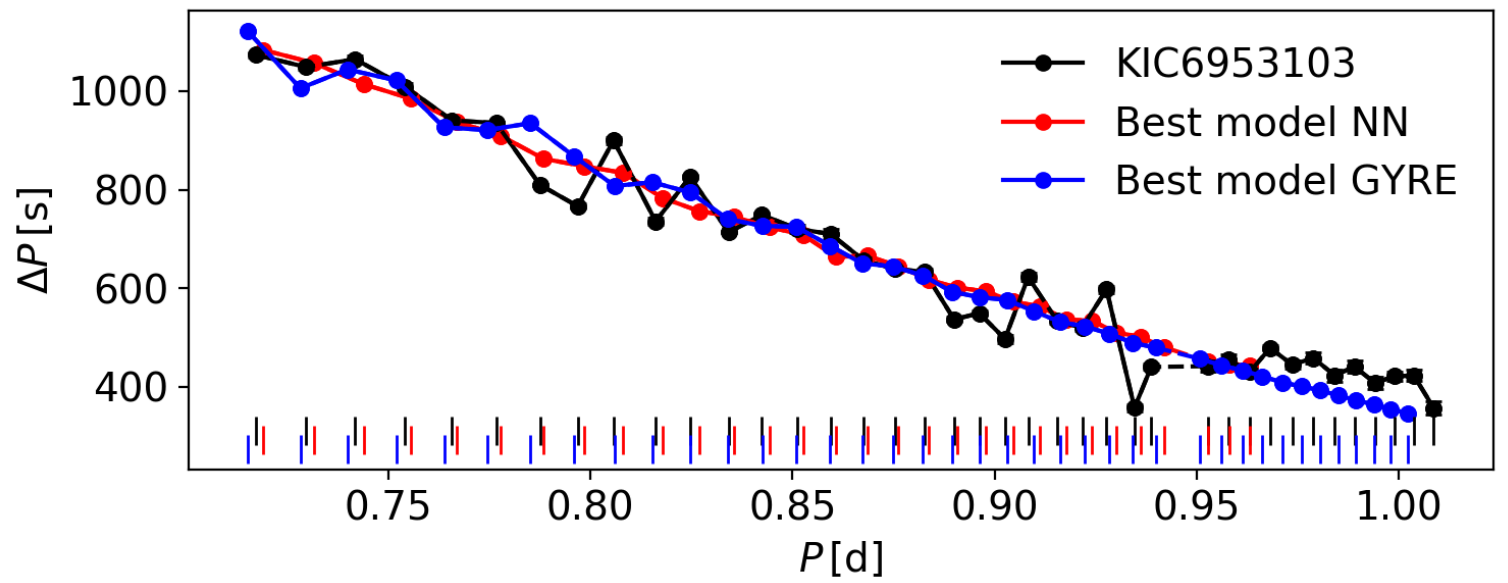

Fig. D.13. Best-fitting models from the neural network and GYRE for KIC 6953103.

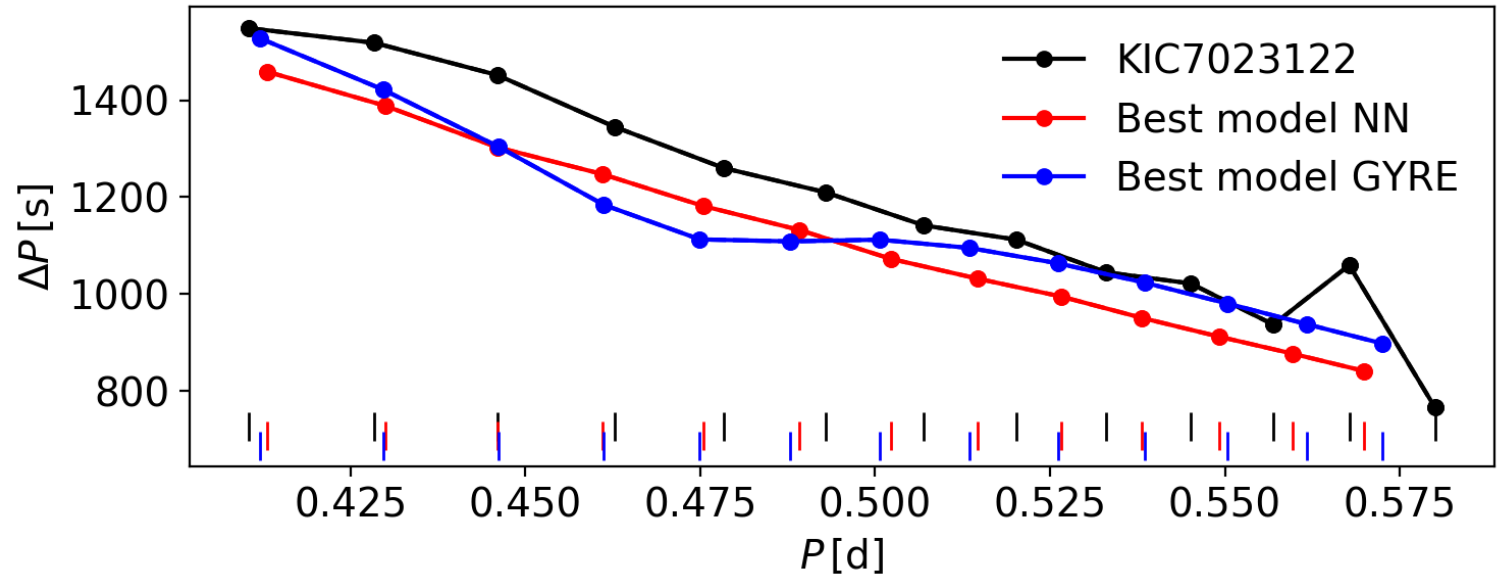

Fig. D.14. Best-fitting models from the neural network and GYRE for KIC 7023122. 


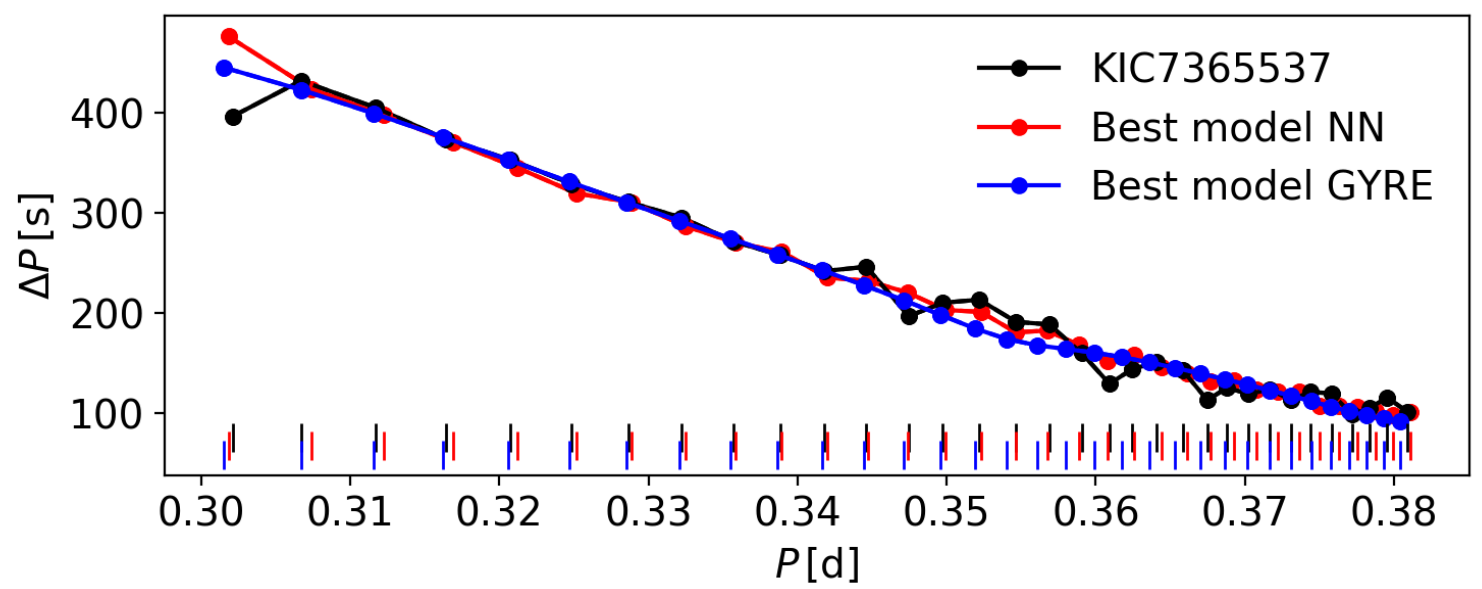

Fig. D.15. Best-fitting models from the neural network and GYRE for KIC 7365537.

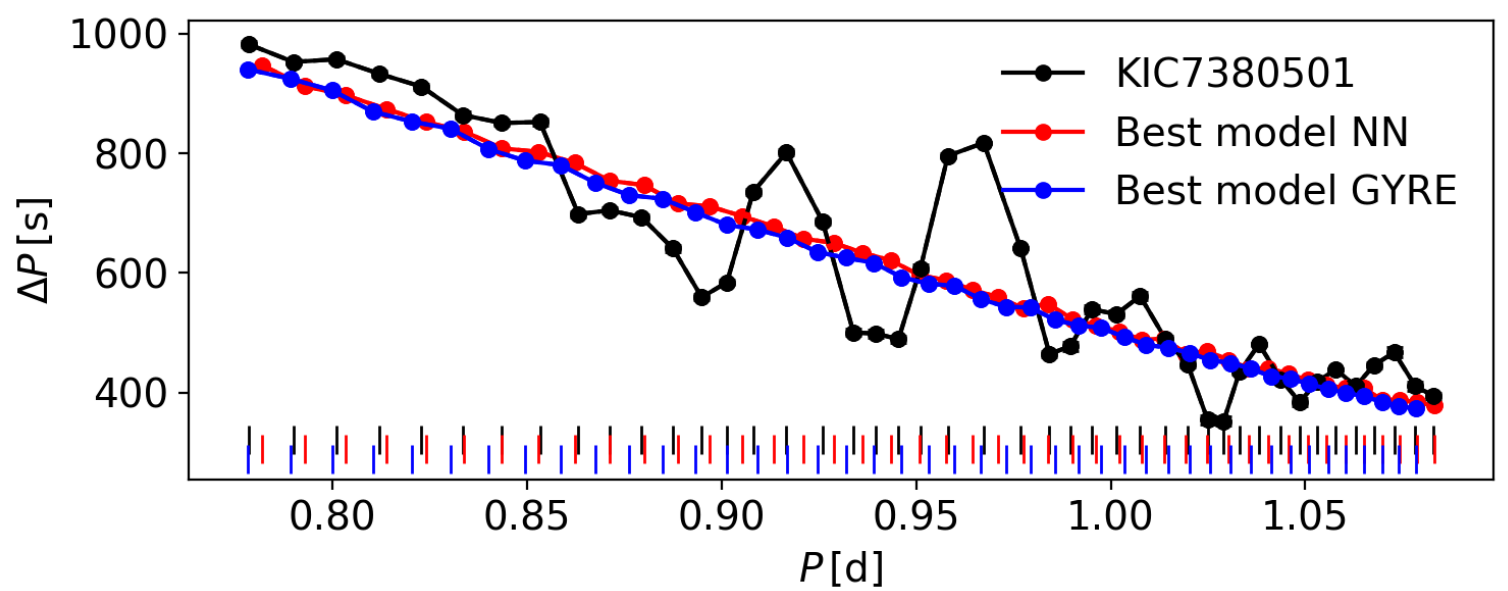

Fig. D.16. Best-fitting models from the neural network and GYRE for KIC 7380501.

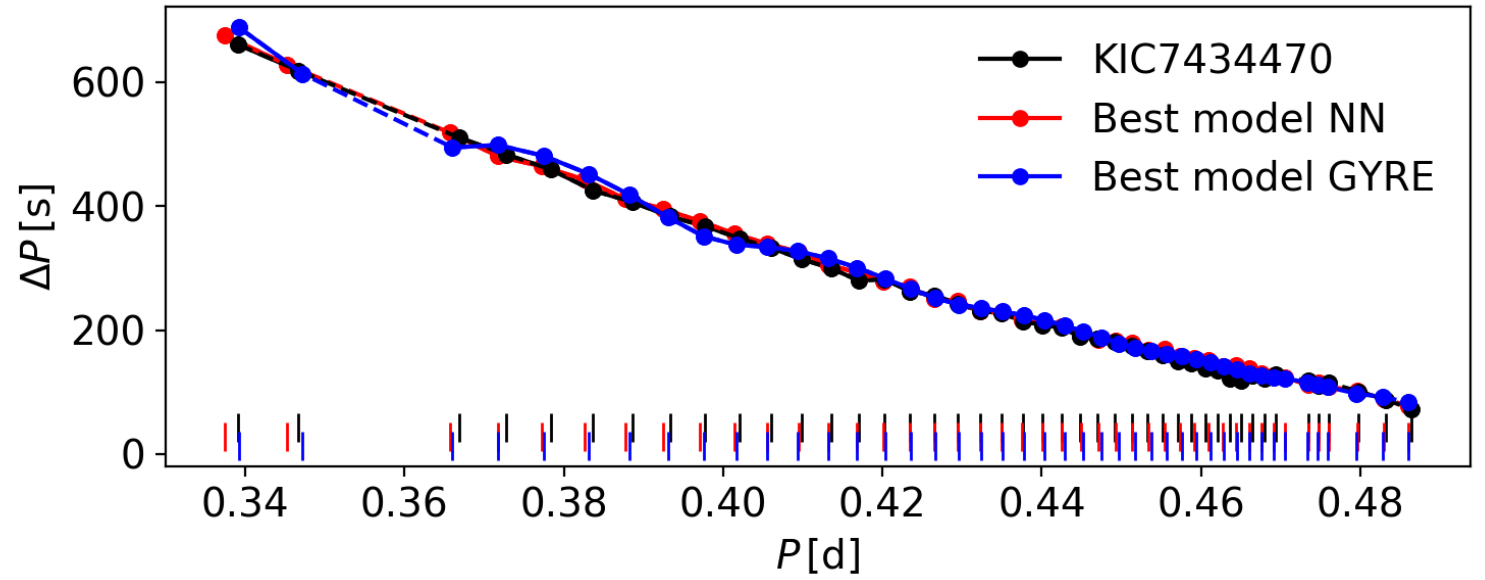

Fig. D.17. Best-fitting models from the neural network and GYRE for KIC 7434470. 


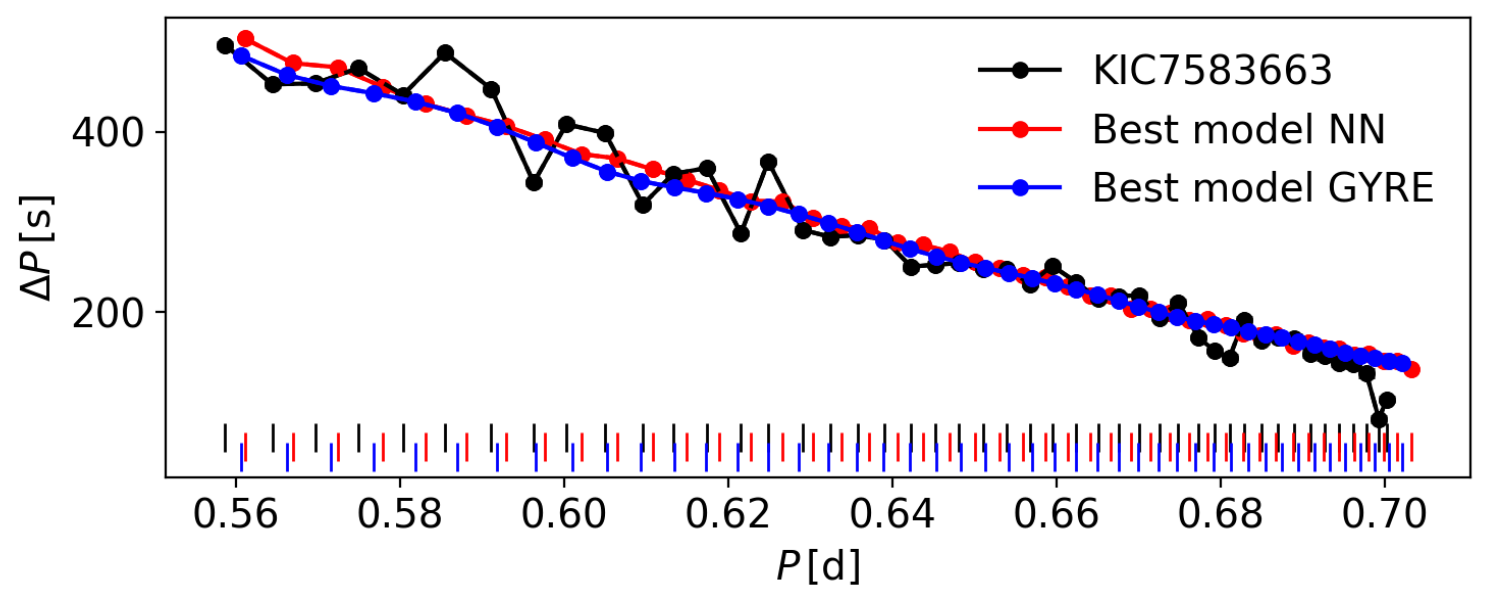

Fig. D.18. Best-fitting models from the neural network and GYRE for KIC 7583663.

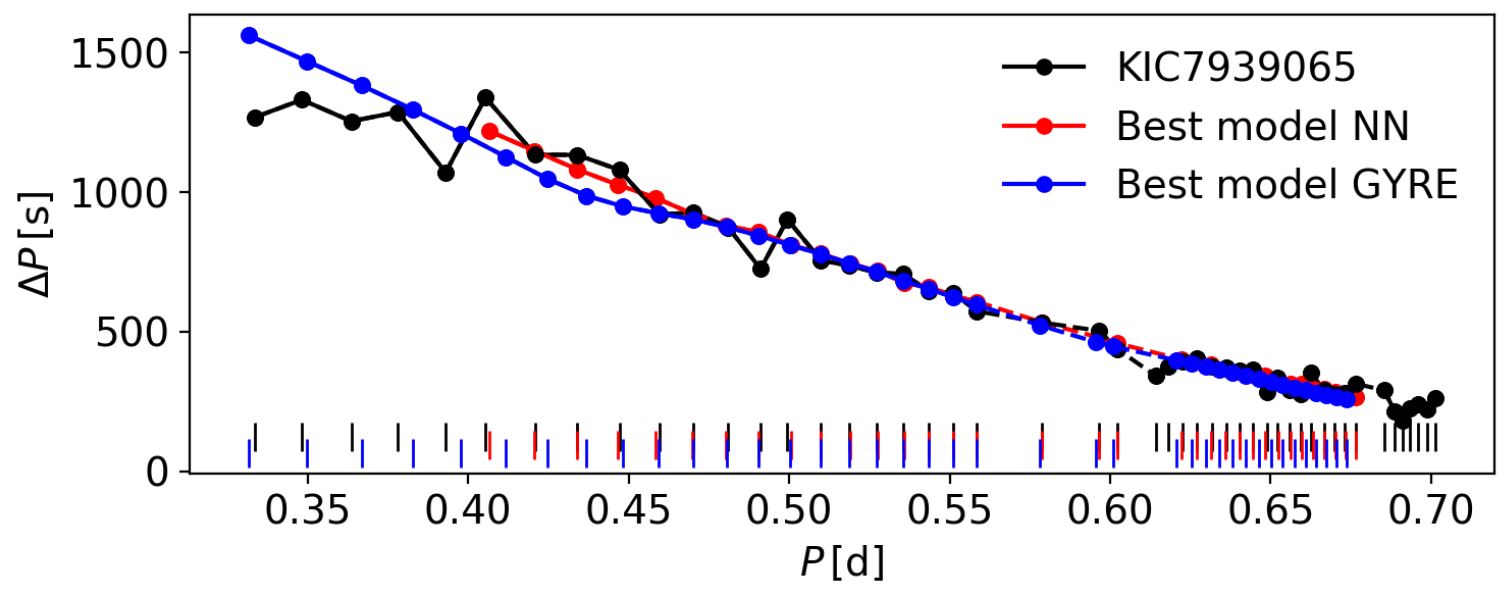

Fig. D.19. Best-fitting models from the neural network and GYRE for KIC 7939065.

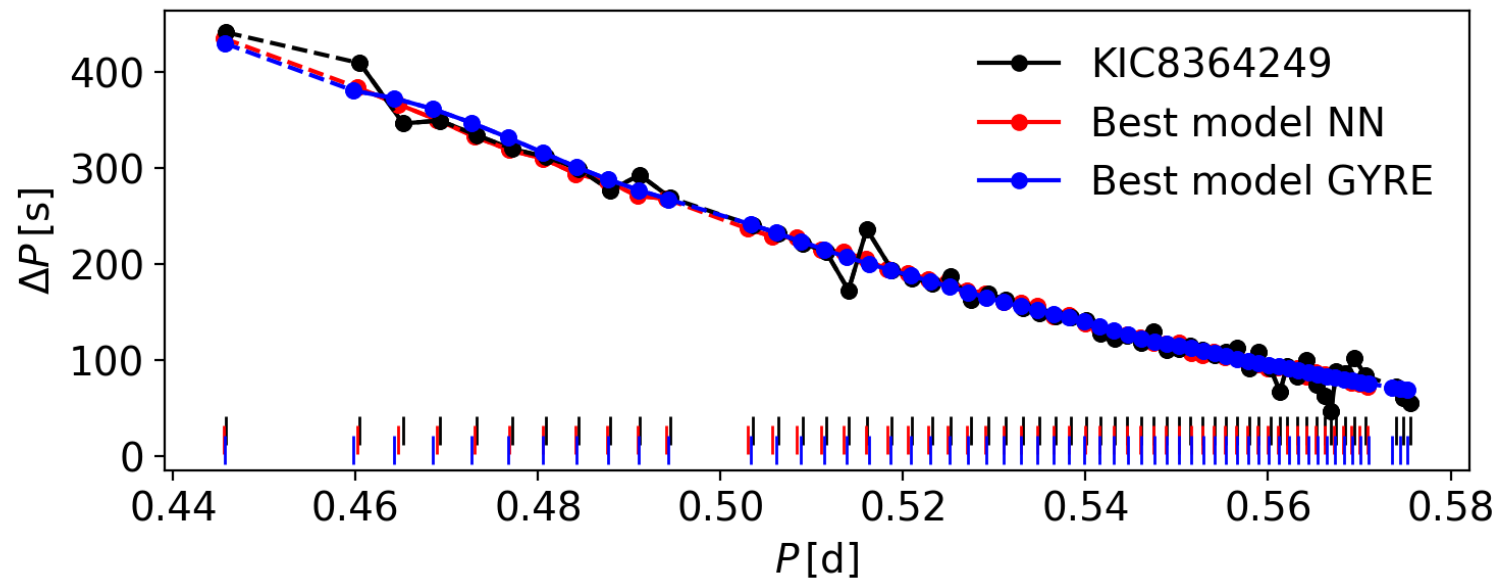

Fig. D.20. Best-fitting models from the neural network and GYRE for KIC 8364249. 


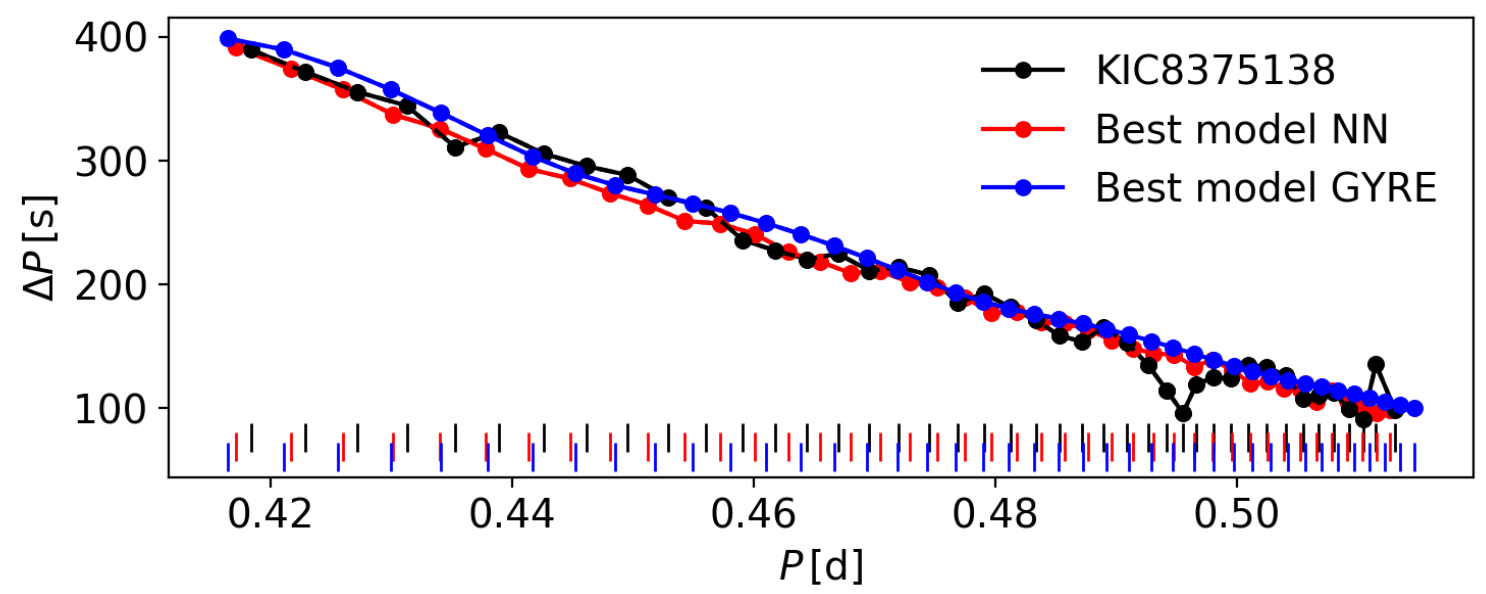

Fig. D.21. Best-fitting models from the neural network and GYRE for KIC 8375138 .

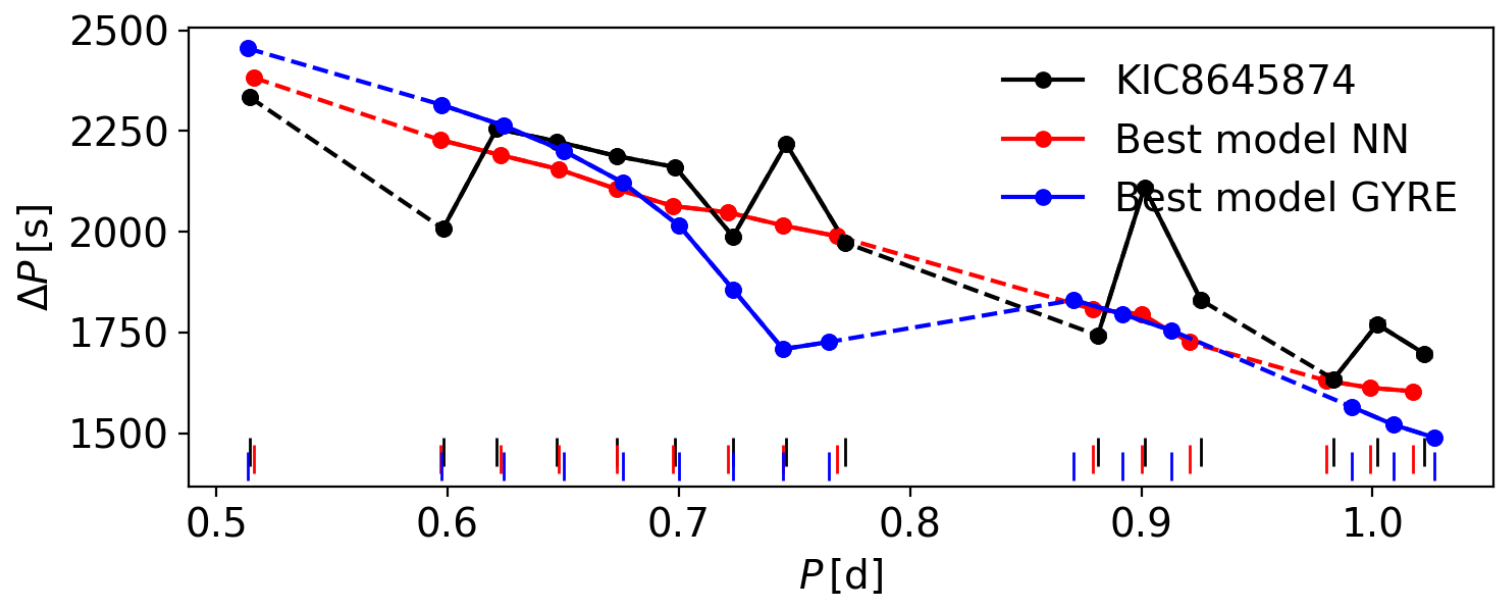

Fig. D.22. Best-fitting models from the neural network and GYRE for KIC 8645874 .

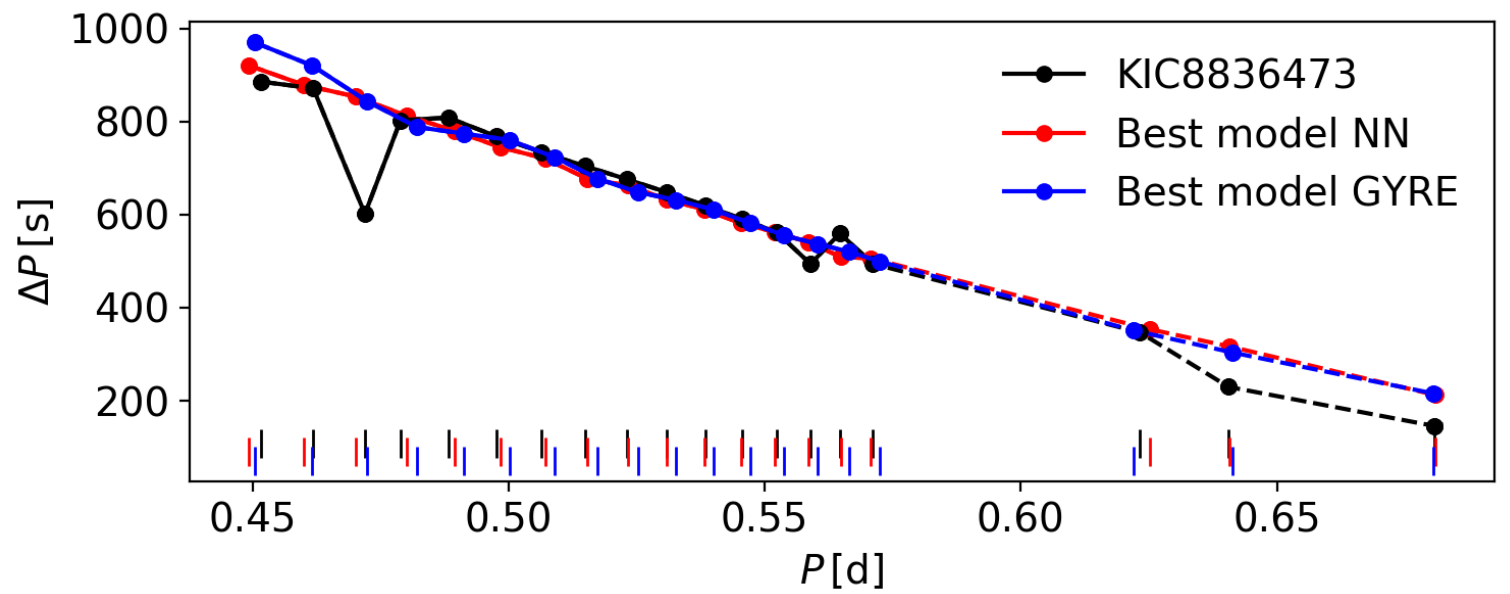

Fig. D.23. Best-fitting models from the neural network and GYRE for KIC 8836473. 


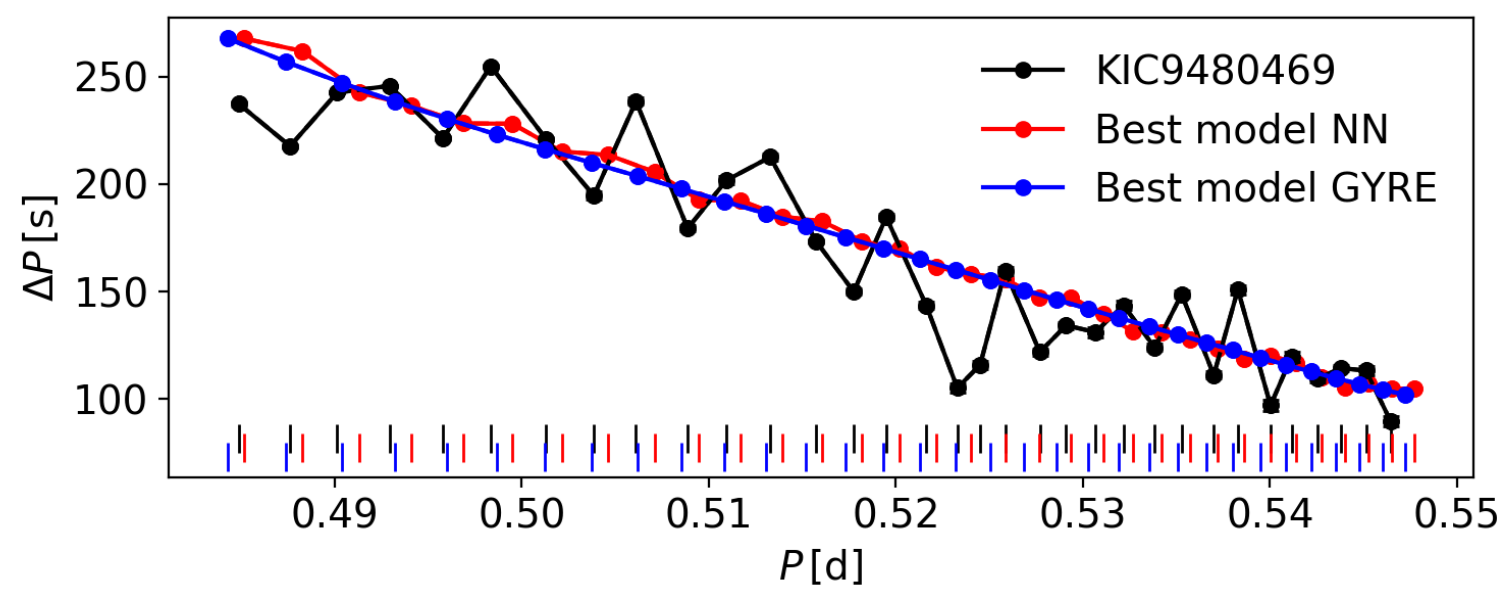

Fig. D.24. Best-fitting models from the neural network and GYRE for KIC 9480469.
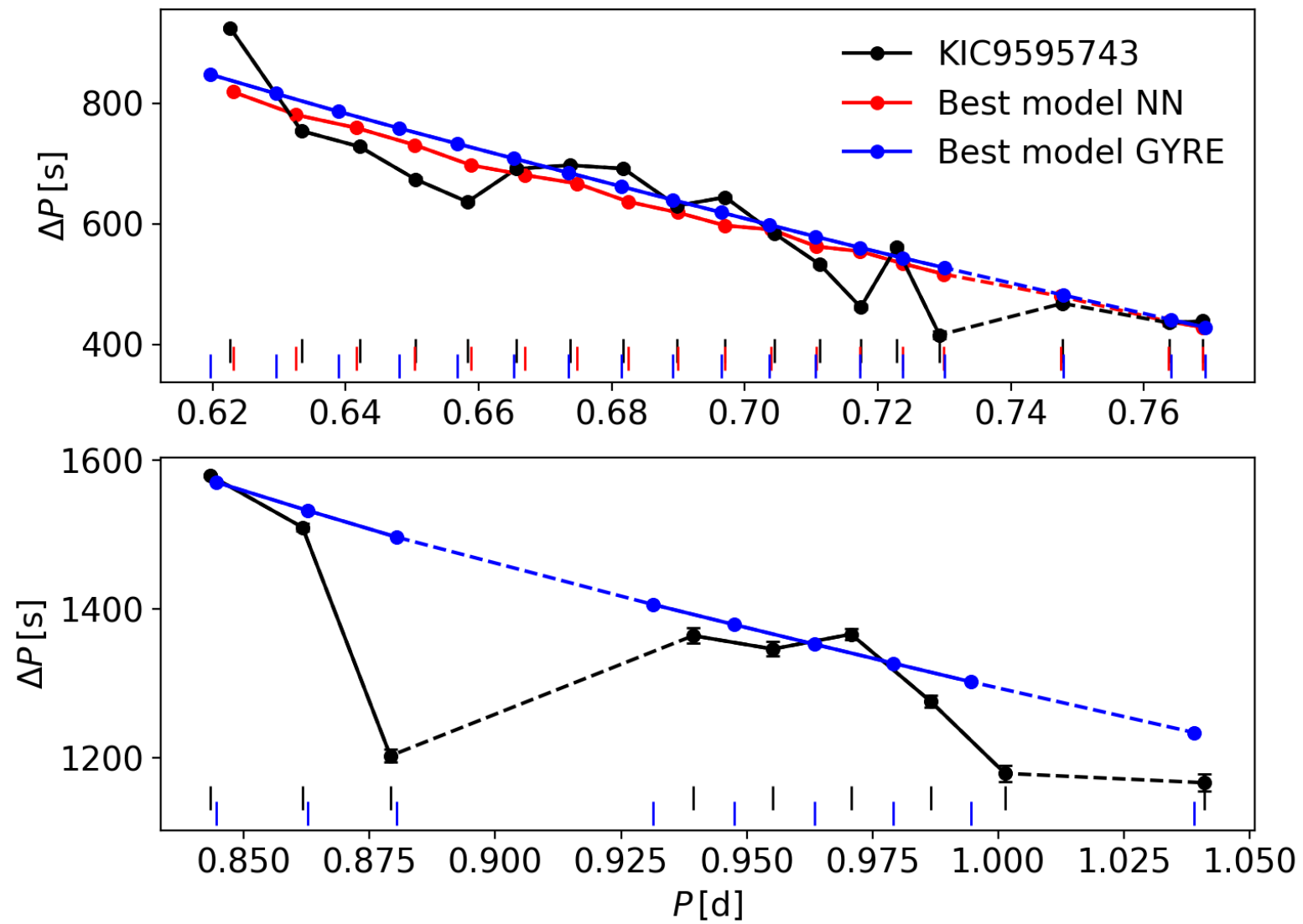

Fig. D.25. Best-fitting models from the neural network and GYRE for KIC 9595743. Top: $(\ell, m)=(1,1)$, bottom $(\ell, m)=(1,0)$. 


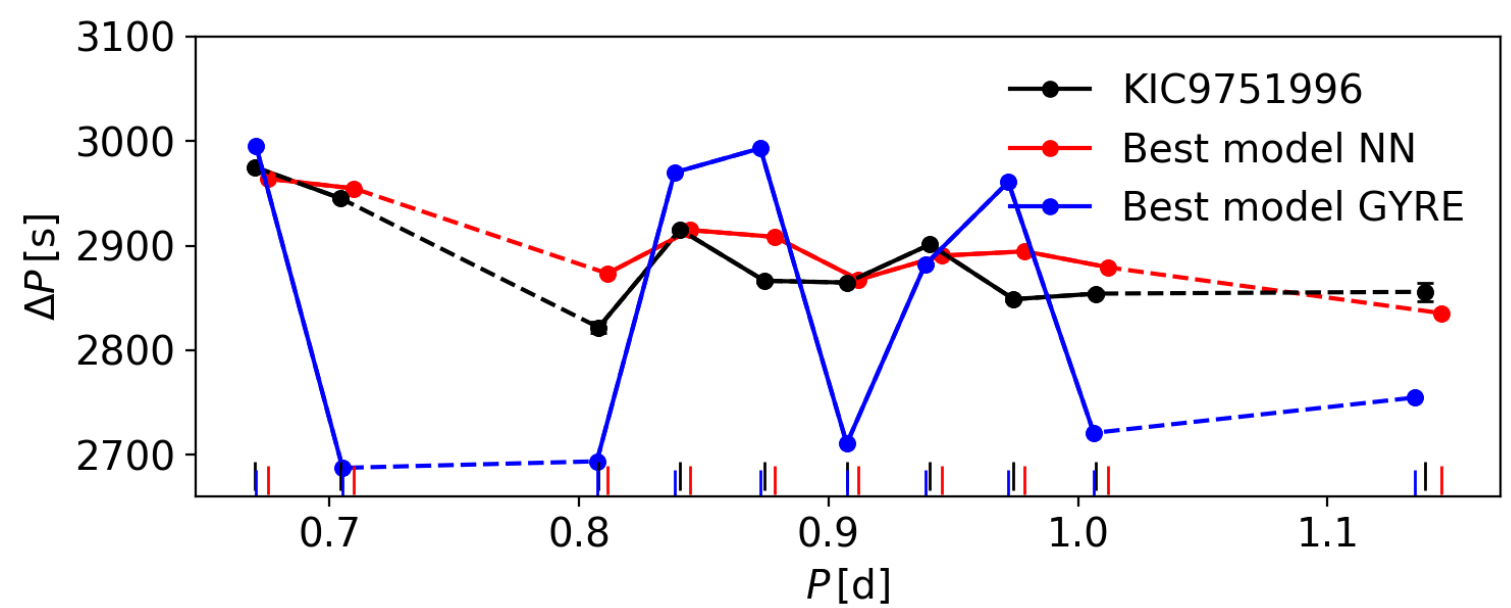

Fig. D.26. Best-fitting models from the neural network and GYRE for KIC 9751996.

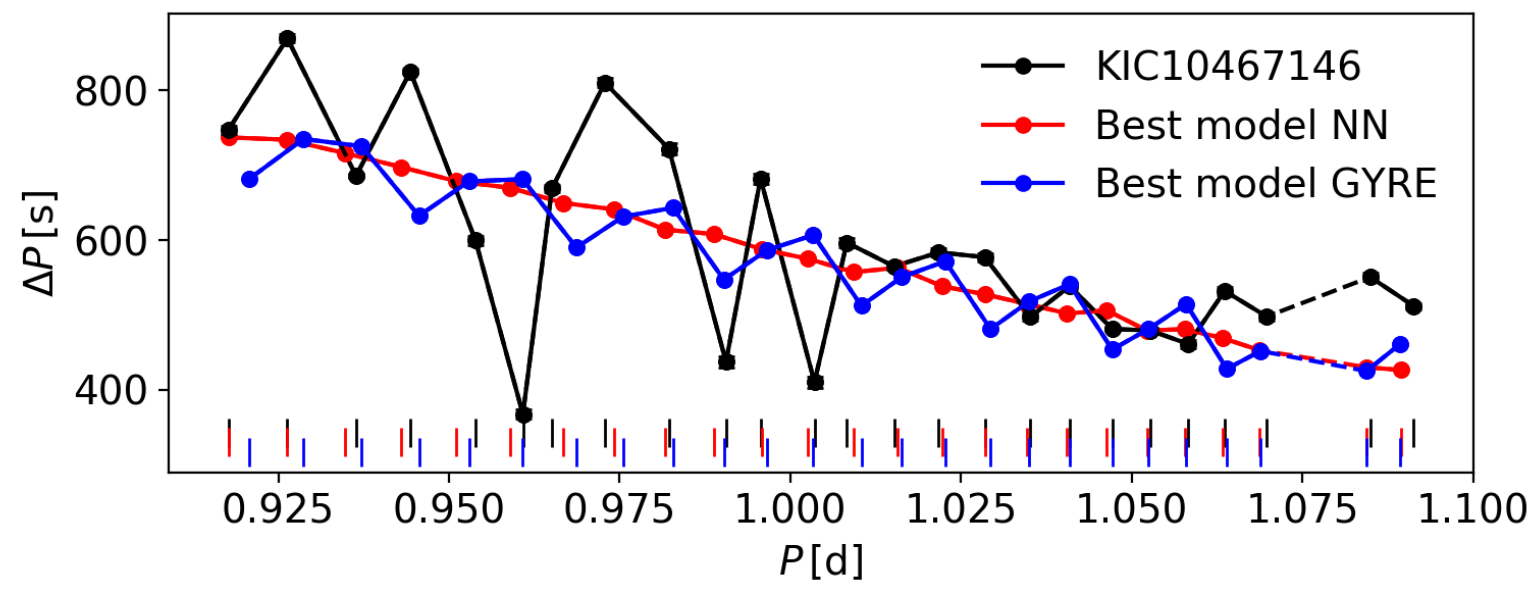

Fig. D.27. Best-fitting models from the neural network and GYRE for KIC 10467146.

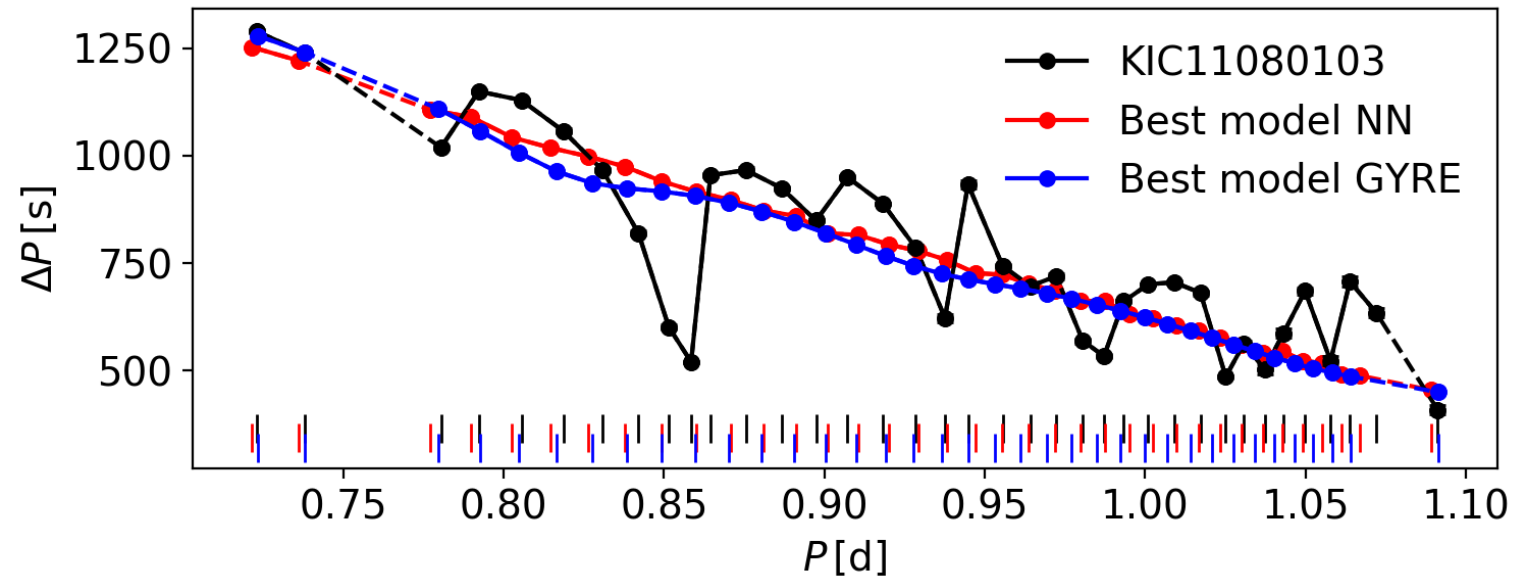

Fig. D.28. Best-fitting models from the neural network and GYRE for KIC 11080103 . 


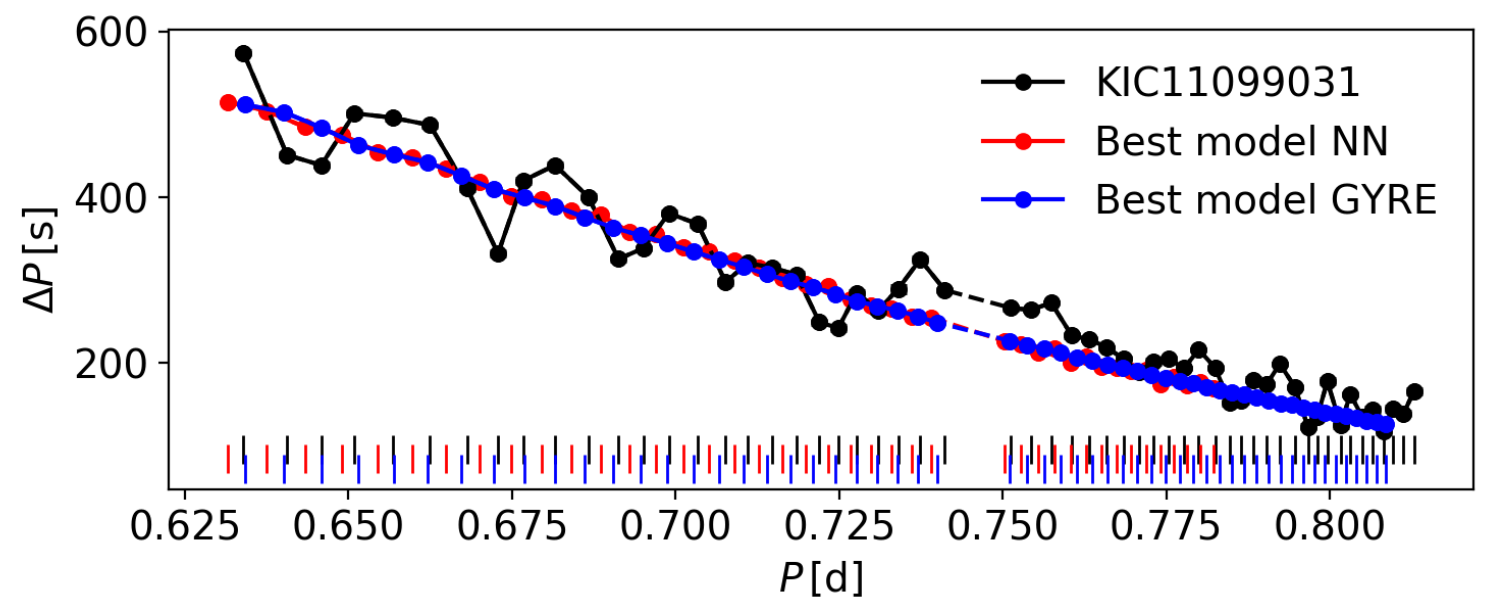

Fig. D.29. Best-fitting models from the neural network and GYRE for KIC 11099031.
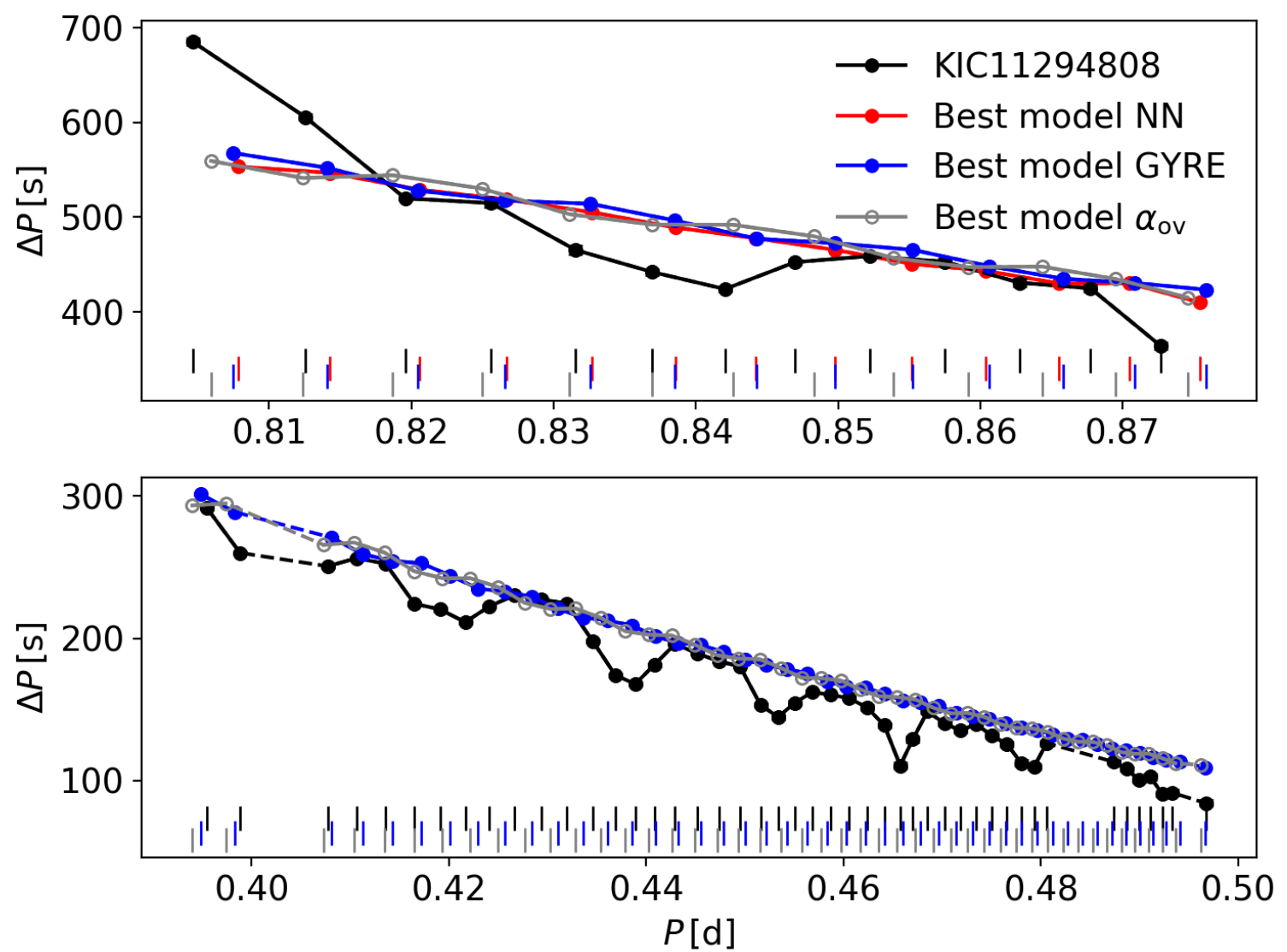

Fig. D.30. Best-fitting models from the neural network and GYRE for KIC 11294808. Top: $(\ell, m)=(1,1)$, bottom: $(\ell, m)=(2,2)$. Additionally, the best-fitting GYRE model based on equilibrium models with a step-like core overshoot prescription is shown in grey. 


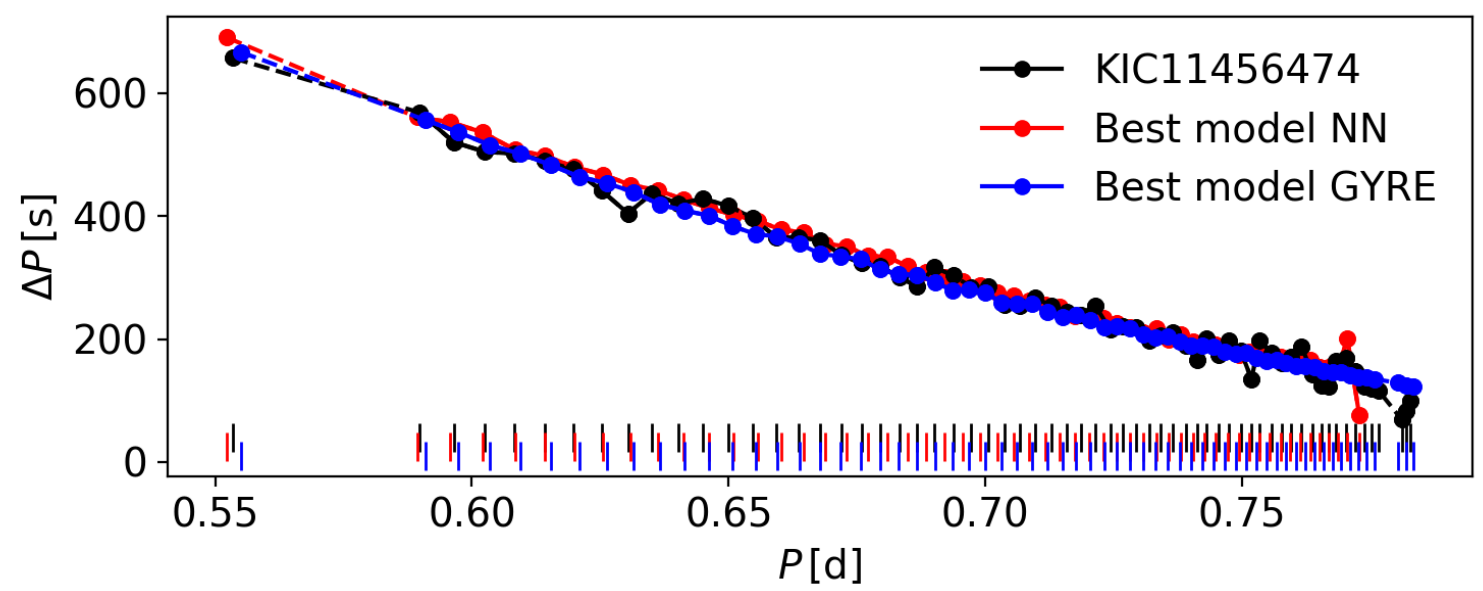

Fig. D.31. Best-fitting models from the neural network and GYRE for KIC 11456474.

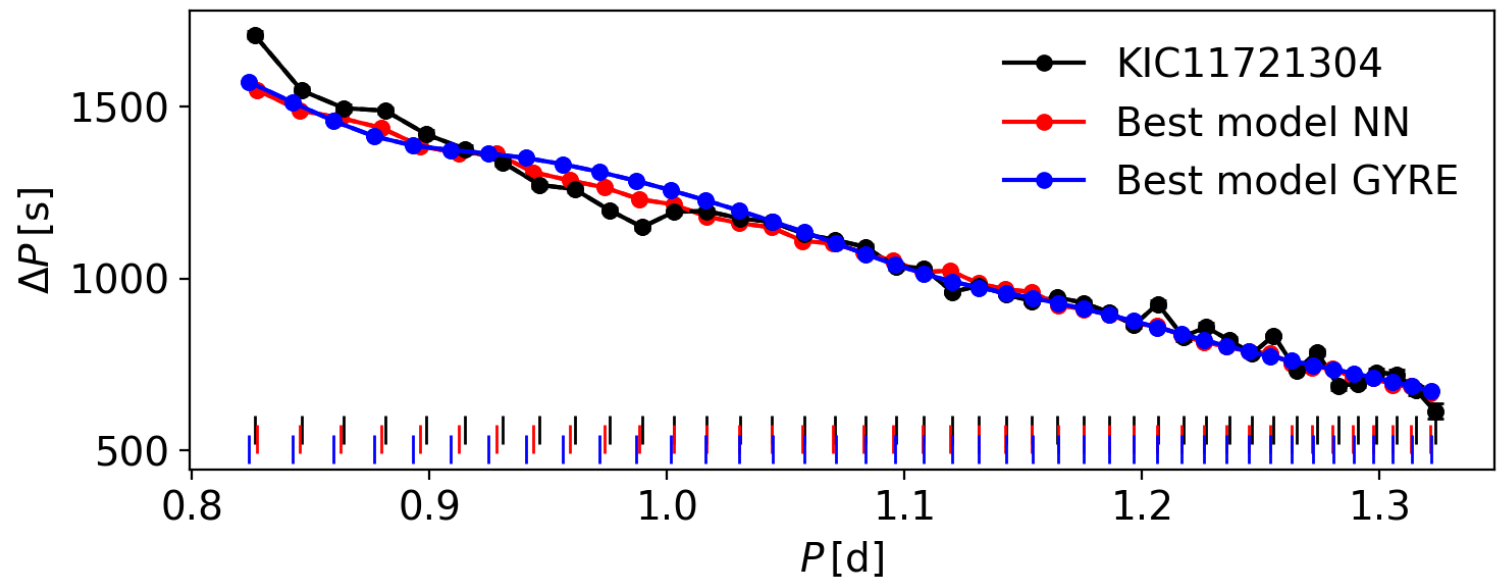

Fig. D.32. Best-fitting models from the neural network and GYRE for KIC 11721304.

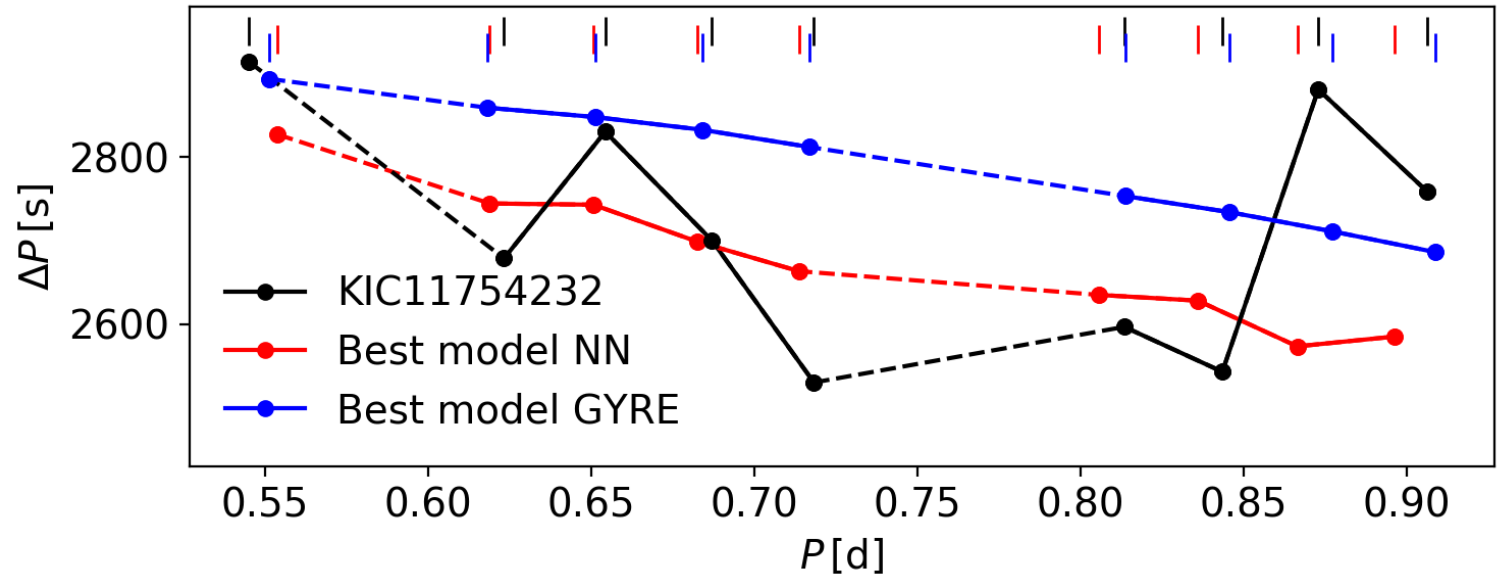

Fig. D.33. Best-fitting models from the neural network and GYRE for KIC 11754232. 


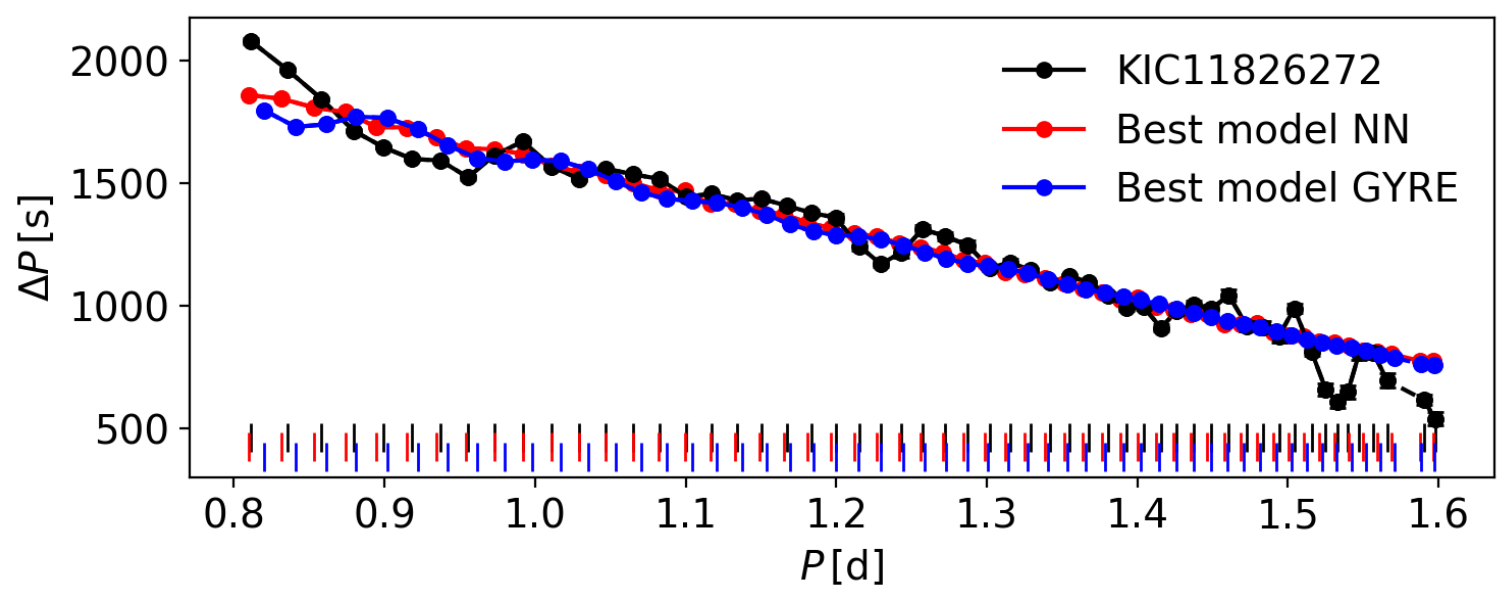

Fig. D.34. Best-fitting models from the neural network and GYRE for KIC 11826272.

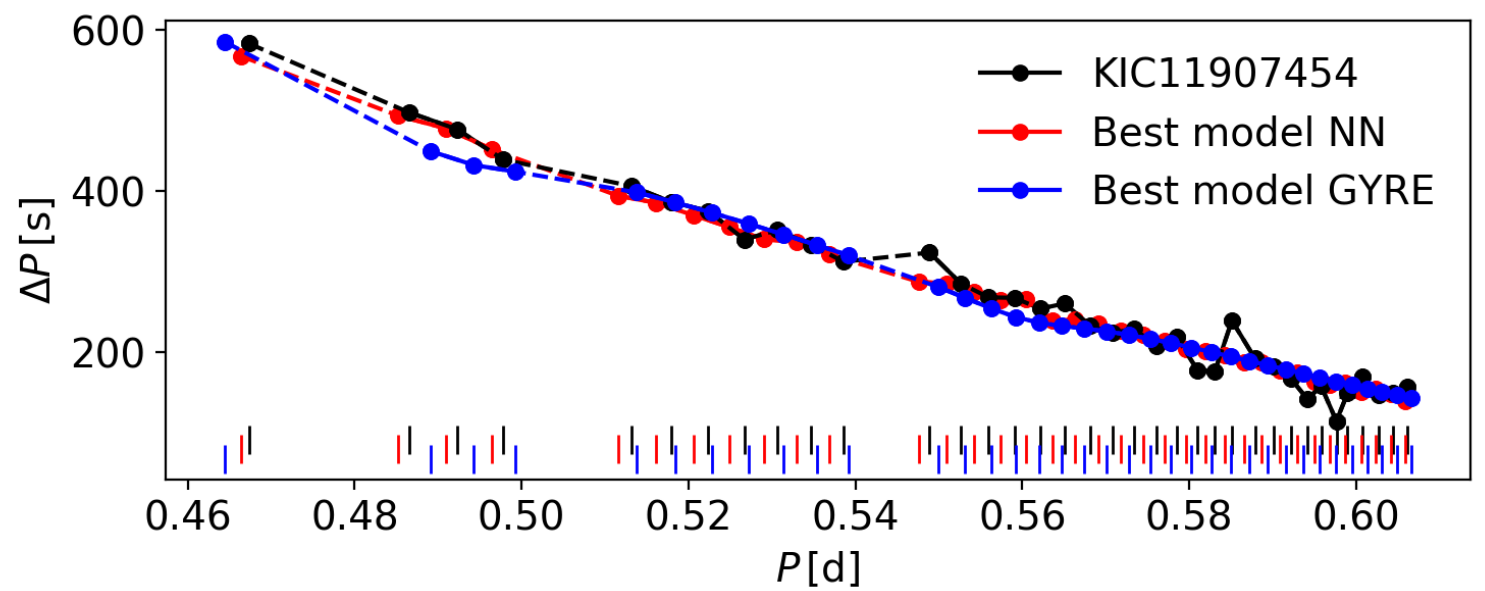

Fig. D.35. Best-fitting models from the neural network and GYRE for KIC 11907454.

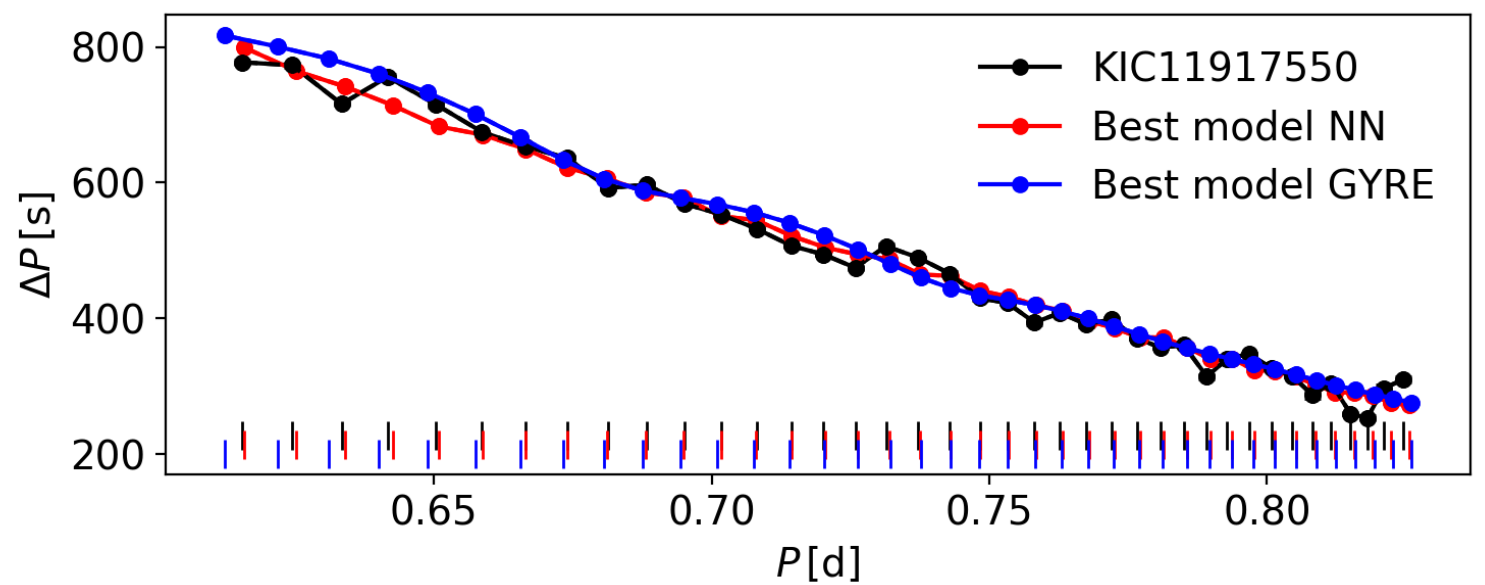

Fig. D.36. Best-fitting models from the neural network and GYRE for KIC 11917550. 


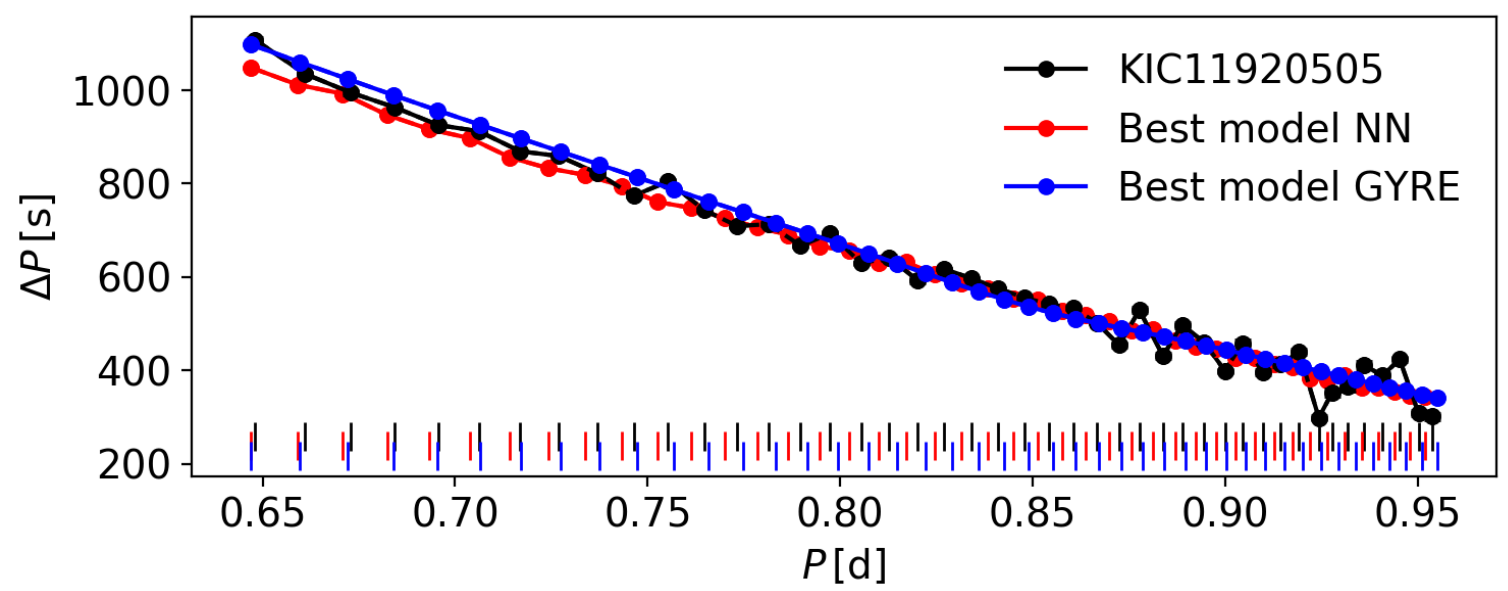

Fig. D.37. Best-fitting models from the neural network and GYRE for KIC 11920505.

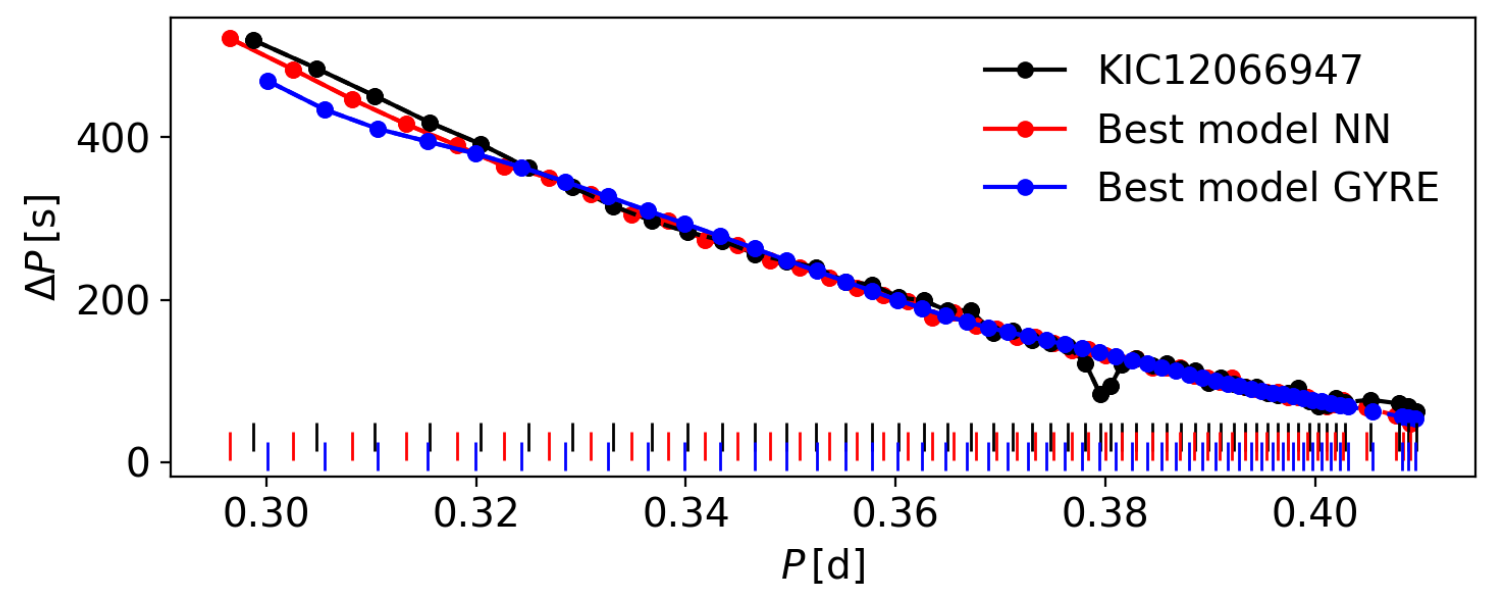

Fig. D.38. Best-fitting models from the neural network and GYRE for KIC 12066947. 


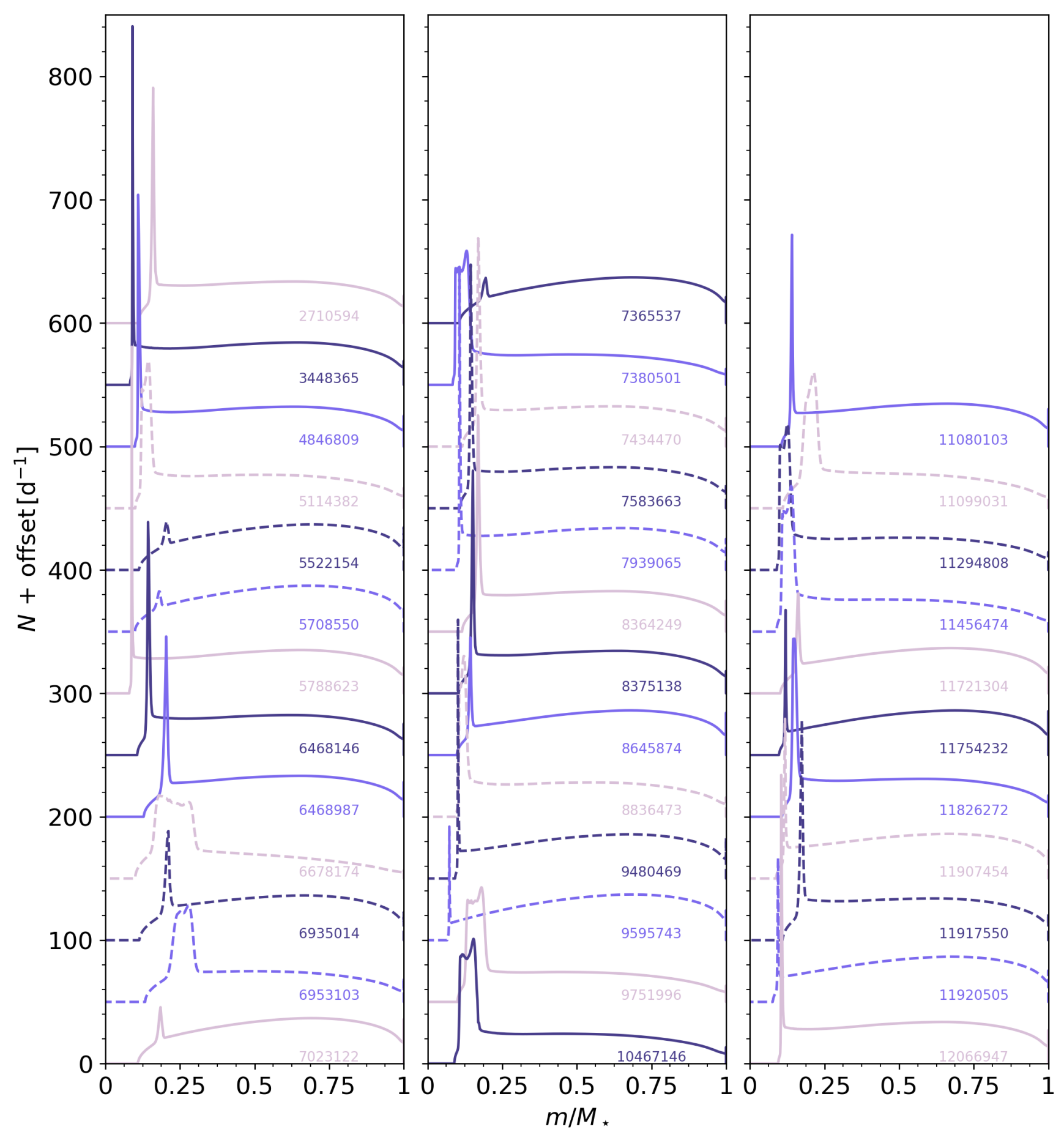

Fig. D.39. Brunt-Väisälä frequency profiles of the best model for each star in our sample. For clarity, the profiles are offset by $50 \mathrm{~d}^{-1}$ from one another. 
Appendix E: Test with $D_{0}=0.05 \mathrm{~cm}^{2} \mathrm{~s}^{-1}$

In the appendix, we show the result of the modelling of KIC 11294808, where we have fixed $D_{0}=0.05 \mathrm{~cm}^{2} \mathrm{~s}^{-1}$ instead of $D_{0}=1 \mathrm{~cm}^{2} \mathrm{~s}^{-1}$ used in the rest of the paper.

\section{Appendix F: The effect of including Gaia}

luminosities on the derived mass

In Figure F.1, we show the difference in inferred stellar mass when we do not include the luminosity from Gaia DR2 in our modelling procedure. 

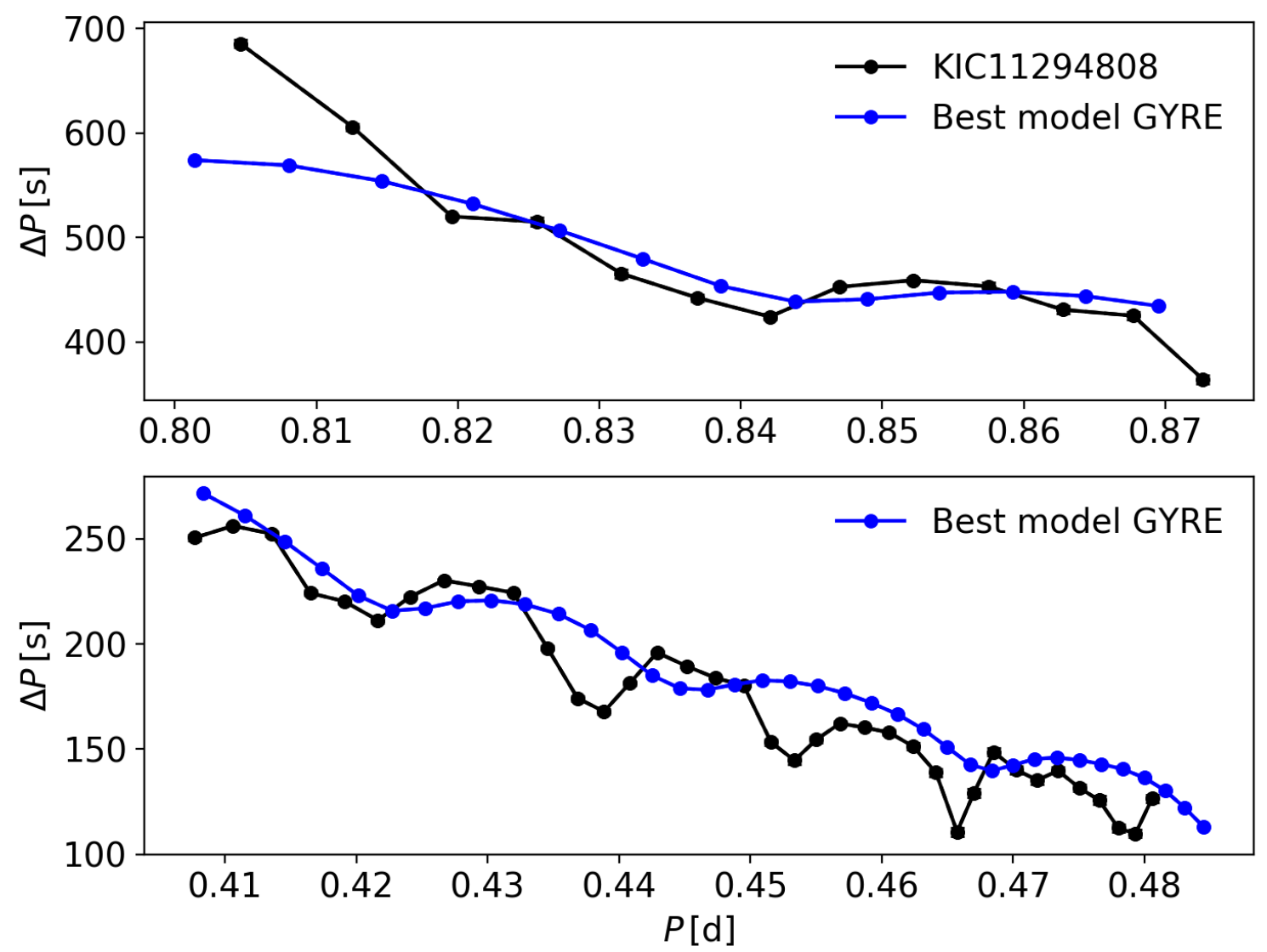

Fig. E.1. Best-fitting GYRE model for KIC 11294808, when $D_{0}=0.05 \mathrm{~cm}^{2} \mathrm{~s}^{-1}$ is assumed. Top: $(\ell, m)=(1,1)$, bottom: $(\ell, m)=(2,2)$.

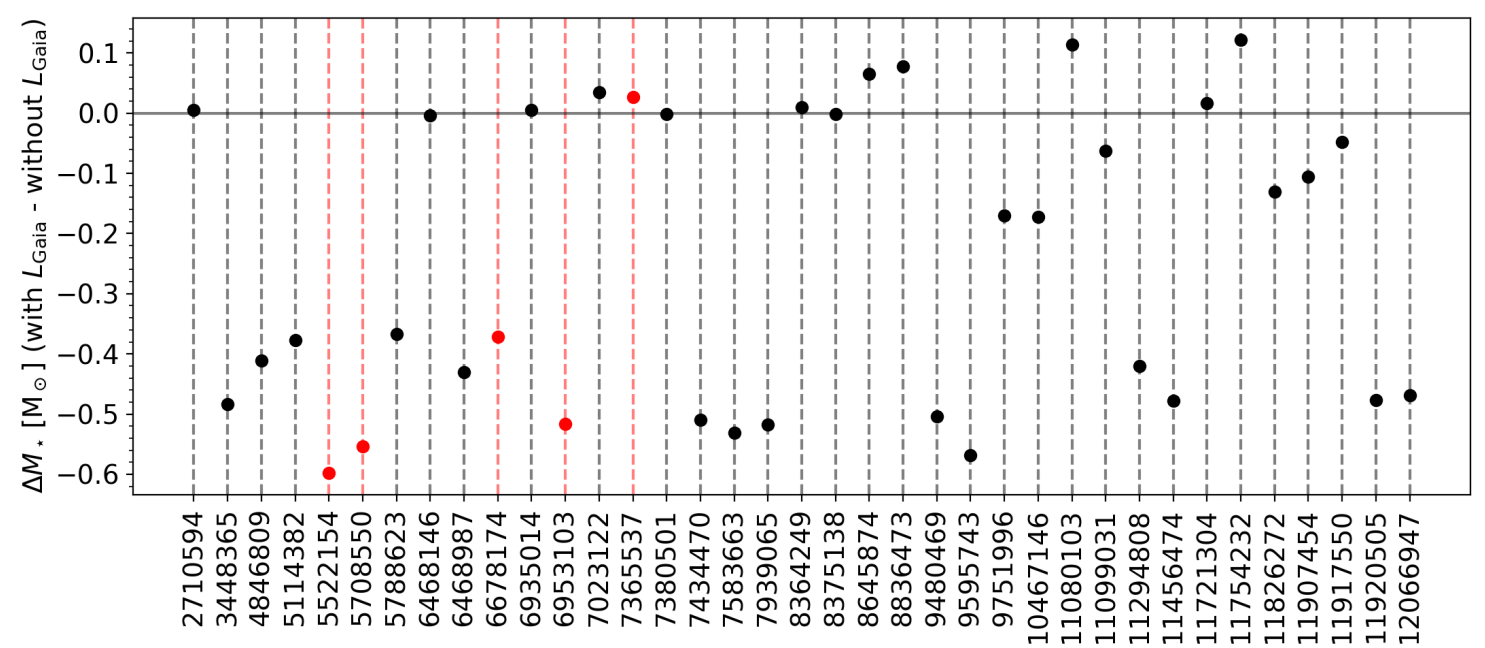

Fig. F.1. Differences in inferred mass per star (KIC ID on the abscissa) when the luminosities of the models are not required to comply with the observed luminosity deduced from Gaia DR2 data. Constraints on $T_{\text {eff }}$ and $\log g$ are in both cases enforced. The stars for which we have not used the Gaia luminosity in the modelling are indicated in red. For these stars, we have put no constraints on the spectroscopic parameters either in our final MLE (hence this solution is not indicated here). 


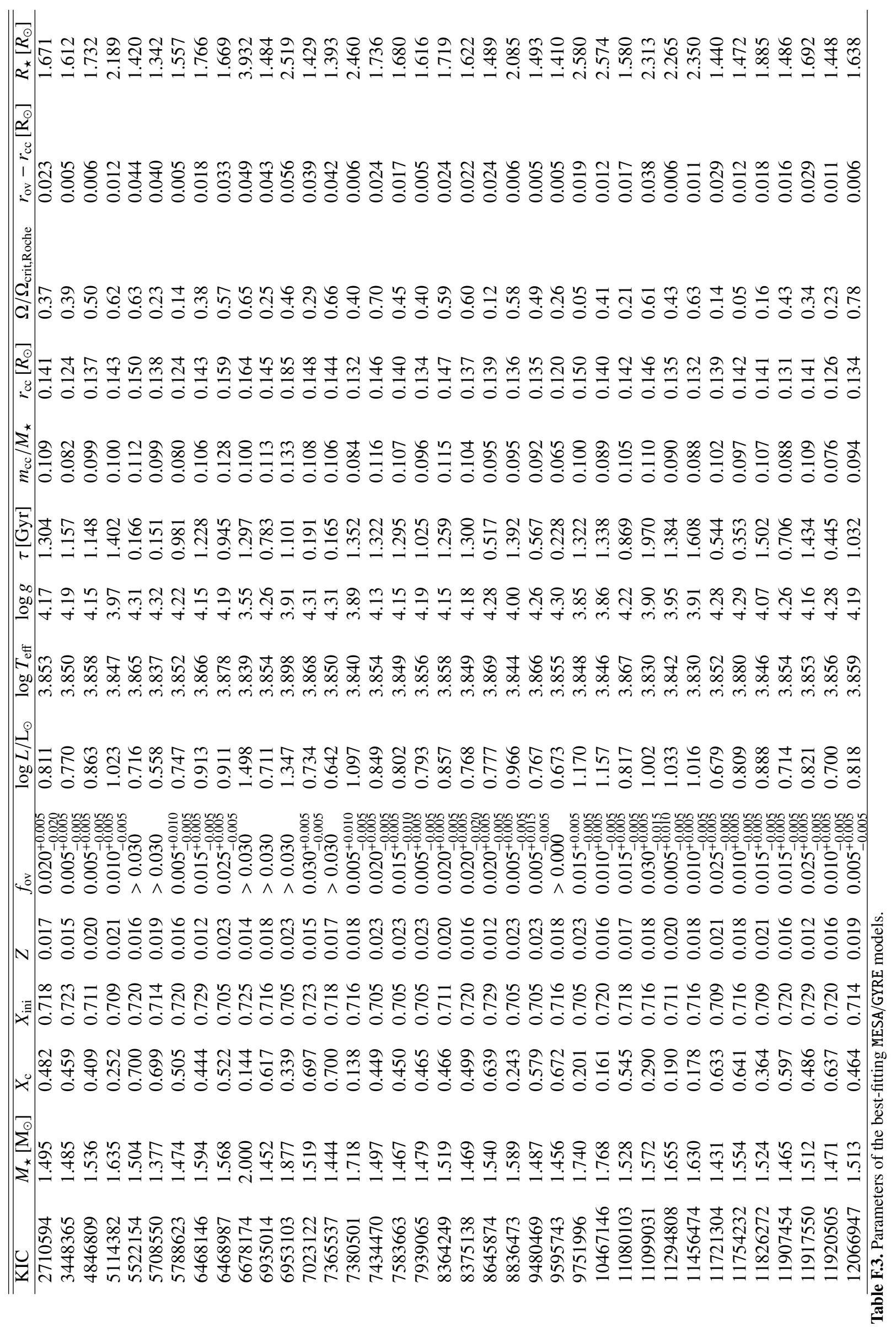

